Supporting Information

\title{
Borenium-Catalyzed Reduction of Pyridines Through the Combined Action of Hydrogen and Hydrosilane
}

\author{
Joshua J. Clarke, ${ }^{\dagger}$ Yuuki Maekawa, ${ }^{\dagger}$ Masakazu Nambo, ${ }^{\Uparrow}$ Cathleen M. Crudden ${ }^{\dagger \uparrow *}$
}

†Department of Chemistry, Queen's University, 90 Bader Lane, Kingston, Ontario, Canada, K7L 3N6

IInstitute of Transformative Bio-Molecules (WPI-ITbM) Nagoya University Furo, Chikusa, Nagoya 464-8602, Japan

Email: cruddenc@chem.queensu.ca

\section{Table of Contents}

1. General Remarks

2. Optimization of Reaction Conditions

3. Substrate Spectroscopic Data

4. Reduction of Pyridines

5. Control Experiments

6. Mechanistic Studies

7. References

8. ${ }^{1} \mathrm{H}$ and ${ }^{13} \mathrm{C}$ NMR Spectra of Products 


\section{General remarks}

\section{Synthesis and techniques}

All preparative scale reactions were conducted in oven dried $\left(160^{\circ} \mathrm{C}\right)$ glassware with magnetic stirring using Schlenk-line techniques or in a glove box under an atmosphere of dry dinitrogen if not mentioned otherwise. Experiments on NMR tube scale were carried out in Teflon cap sealed NMR tubes (ø 5mm). Solvents were purified by passage over an activated aluminum oxide column, followed by distillation from Na-benzophenone ketal where necessary (toluene, benzene, THF, hexanes, pentane) and degassed prior to use. Dichloromethane, $\mathrm{CD}_{2} \mathrm{Cl}_{2}$, and $\alpha, \alpha, \alpha$-trifluorotoluene were distilled from $\mathrm{CaH}_{2}$ (followed by 3 freezepump-thaw cycles and stored over a mixture of $4 \AA$ molecular sieves). Toluene- $\mathrm{d}_{8}$ and benzene- $\mathrm{d}_{6}$ were degassed by 3 freeze-pump-thaw cycles and stored over activated $4 \AA$ molecular sieves. DMF was of DrySolv-quality and used as received. Solvents for chromatography and other syntheses were used as received from commercial sources and were at least of ACS reagent grade. Solvents for routine NMR spectroscopy experiments were used as received. Silica gel 60 (particle size $0.040-0.063 \mathrm{~mm}, 230-400$ mesh) was purchased from Silicycle. TLCs were carried out on silica gel coated aluminum plates with UV indicator (F254) obtained by EMD Chemicals, Inc. and analyzed by UV/VIS and stained using a cerium ammonium molybdate solution.

\section{Reagents and materials}

Reagents for piperidine synthesis were used as received without further purification unless noted otherwise. 9-Borabicyclo[3.3.1]nonane dimer, and sodium bis(trimethylsilyl)amide (NaHMDS) were purchased from Aldrich, stored in a glove box, and used as received. 2-Bromopyridine, 2,6-lutidine, and 2-phenylpyridine were purchased from Aldrich, distilled from $\mathrm{CaH}_{2}$ (followed by 3 freeze-pump-thaw cycles and stored over a mixture of $4 \AA$ molecular sieves) and stored in a glove box. $\mathrm{Ph}_{3} \mathrm{C}^{+} \mathrm{B}\left(\mathrm{C}_{6} \mathrm{~F}_{5}\right)_{4}{ }^{-}$, was purchased from Strem stored in a glove box, and used as received. 2-Bromo-6-methylpyridine was purchased from Oakwood Chemicals and used as received. Aryl boronic acids were purchased from Aldrich, Frontier Scientific or Acros Chemicals and used as received. Pyridine $\mathbf{7 h}$ was synthesized using a published procedure. ${ }^{1}$ All silanes in this study were purchased from Aldrich, distilled from $\mathrm{CaH}_{2}$ (followed by 3 freeze-pump-thaw cycles and stored over a mixture of $4 \AA$ molecular sieves) and stored in a glove box before use. Palladium (II) acetate, benzoic acid and cis-2,6-dimethylpiperidine were purchased from Aldrich and used as received. Potassium tribasic phosphate was purchased from Fischer Scientific and used as received. Diphenyliodonium tetrafluoroborate ${ }^{2}$ and $\mathbf{1}-\mathbf{H}^{3}$ were synthesized via known procedures.

\section{Characterization}


NMR spectra were recorded on Bruker Avance $300\left({ }^{1} \mathrm{H}: 300.13{ }^{13} \mathrm{C}: 75.47\right.$; TXI probe), Bruker Avance $400\left({ }^{1} \mathrm{H}: 400.13,{ }^{11} \mathrm{~B}: 128.38,{ }^{13} \mathrm{C}: 100.62,{ }^{19} \mathrm{~F}: 376.50,{ }^{31} \mathrm{P}: 161.98\right.$; BBI, BBFO and QNP probes), Bruker Avance $500\left({ }^{1} \mathrm{H}: 500.19,{ }^{2} \mathrm{H}: 426.80,{ }^{11} \mathrm{~B}: 160.27,{ }^{13} \mathrm{C}: 125.62\right.$; BBFO probe), Bruker NEO 500 $\left({ }^{1} \mathrm{H}: 500.19,{ }^{2} \mathrm{H}: 426.80,{ }^{11} \mathrm{~B}: 160.27,{ }^{13} \mathrm{C}: 125.62\right.$; BBFO probe), Bruker Avance $600\left({ }^{1} \mathrm{H}: 600.17,{ }^{11} \mathrm{~B}\right.$ : 192.56, ${ }^{13} \mathrm{C}: 150.93 ;{ }^{31} \mathrm{P}: 242.94$,TBI probe) or Bruker NEO $700\left({ }^{1} \mathrm{H}: 700.13,{ }^{11} \mathrm{~B}: 224.63,{ }^{13} \mathrm{C}: 176.05\right.$, ${ }^{31} \mathrm{P}: 283.42$; BBFO probe) instruments operating at the denoted spectrometer frequency given in mega Hertz $(\mathrm{MHz})$ for the specified nucleus. The samples were measured as solutions in the stated solvent at ambient temperature in non-spinning mode if not mentioned otherwise. To specify the signal multiplicity, the following abbreviations are used: $\mathrm{s}=$ singlet, $\mathrm{d}=$ doublet, $\mathrm{t}=$ triplet, $\mathrm{q}=$ quartet, $\mathrm{qu}=$ quintet, $\mathrm{sept}=$ septet, oct $=$ octet, and $\mathrm{m}=$ multiplet; br. indicates a broad resonance. Shifts $\delta$ are reported in parts per million (ppm) relative to tetramethylsilane (TMS) as an external standard for ${ }^{1} \mathrm{H}$ and ${ }^{13} \mathrm{C}$ NMR spectra and calibrated against the solvent residual peak or in case of proteo-solvents against known solvent resonances. ${ }^{11} \mathrm{~B}$ signals are calibrated against external $\mathrm{BF}_{3} \cdot \mathrm{OEt}_{2}$ and ${ }^{19} \mathrm{~F}$ against $\mathrm{CFCl}_{3}$. Coupling constants $\mathrm{J}$ are given in Hertz (Hz). GC-MS measurements were performed on an Agilent Technologies GC 6850N/ MS 5975N VL MSD equipped with an Agilent Technologies HP-5MS column (length: 30m, 0.25 mm inner diameter, $0.25 \mu \mathrm{m}$ coating thickness) coupled to a quadrupole mass filter. Helium was used as the carrier gas with a constant flow of $1.2 \mathrm{~mL} / \mathrm{min}$. Separation of the injected species was achieved using the denoted temperature program and retention times $t_{R}$ are given in minutes (min). High resolution mass-spectra (HRMS) were measured by the Queen's Mass Spectrometry and Proteomics Unit (MSPU) at Queen's University, Kingston, Ontario, Canada. Mass spectra were measured on Applied Biosystems/MDS Sciex QStar XL QqTOF or Waters ZQ Single Quad. Fragment signals are given in mass per charge number (m/z). 


\section{Optimization of Reaction Conditions}

Table S1. Solvent optimization of $\mathbf{1}^{+}$-catalyzed hydrogenation of 2,6-diphenylpyridine

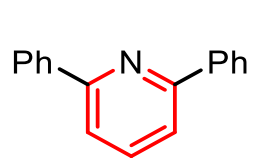

3

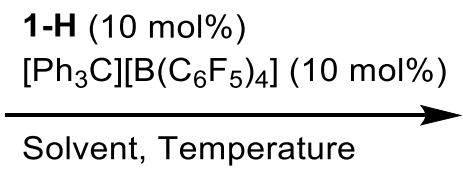

$\mathrm{H}_{2}$ (47 bar)<smiles>Pc1ccccc1</smiles>

4

$\begin{array}{cccc}\text { Entry } & \text { Solvent } & \text { Temperature }\left({ }^{\circ} \mathrm{C}\right) & \text { Yield }^{\mathrm{a}} \text { (Conversion) } \\ 1 & \text { Dioxane } & 110 & \text { No reaction } \\ 2 & \text { 1,2-dichloroethane } & 90 & 20(21) \\ 3 & \mathrm{C}_{6} \mathrm{H}_{5} \mathrm{CF}_{3} & 110 & \text { No reaction } \\ 4 & \text { Toluene } & 120 & 31(38)\end{array}$

All reactions were carried out on $0.125 \mathrm{mmol}$ scale of 3 . ${ }^{\text {a Based on crude }}{ }^{1} \mathrm{H}$ NMR analysis compared to 1,3,5-trimethoxybenzene as internal standard.

Table S2. Temperature optimization of $\mathbf{1}^{+}$-catalyzed hydrogenation of 2,6-diphenylpyridine<smiles>c1ccc(-c2ccccn2)cc1</smiles>

3

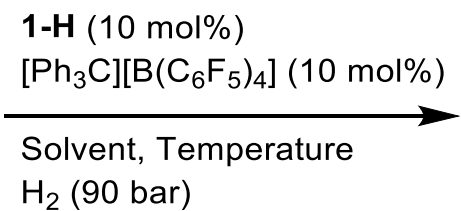

$\mathrm{H}_{2}$ (90 bar)<smiles>CC1CCC(c2ccccc2)NC1c1ccccc1</smiles>

4

$\begin{array}{cccc}\text { Entry } & \text { Solvent } & \text { Temperature }\left({ }^{\circ} \mathrm{C}\right) & \text { Yield }^{\text {b }} \text { (Conversion) } \\ 1 & \text { Toluene } & 60 & 36(33) \\ 2 & \text { Toluene } & 90 & 40(49) \\ 3 & \text { Toluene } & 120 & 40(41) \\ 4 & \text { DCE } & 60 & 48(43) \\ 5 & \text { DCE } & 90 & 40(41) \\ 6^{\text {a }} & \text { DCE } & 60 & 48(63)\end{array}$

All reactions were carried out on $0.125 \mathrm{mmol}$ scale of $\mathbf{3}^{\mathrm{a}} 20 \mathrm{~mol} \%$ of $\mathbf{1 - H}$ and $\left[\mathrm{Ph}_{3} \mathrm{C}\right]\left[\mathrm{B}\left(\mathrm{C}_{6} \mathrm{~F}_{5}\right)_{4}\right]^{\mathrm{b}} \mathrm{Based}$ on crude ${ }^{1} \mathrm{H}$ NMR analysis compared to 1,3,5-trimethoxybenzene as internal standard. 


\section{Substrate Spectroscopic Data}

7a-7f, 7i, and 9b-9d were synthesized using reported methods [Eur. J. Org. Chem. 2010, 29, 5548].

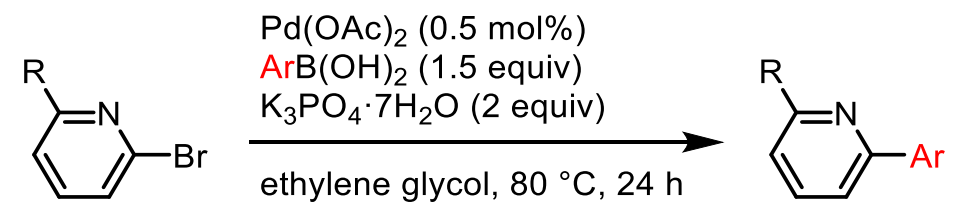

\section{2-Methyl-6-phenylpyridine (7a)}<smiles>Cc1cccc(-c2ccccc2)n1</smiles>

Spectra match literature: Paterson, A. J., Heron, C. J., McMullen, C. L., Mahon, M. F., Press, N. J., and Frost, C. G., Org. Biomol. Chem. 2017, 15, 5993-6000

Physical description: white solid

${ }^{1}$ H NMR (400 MHz, $\left.\mathbf{C D C l}_{3}\right) \delta 7.98(\mathrm{~d}, 2 \mathrm{H}, J=8.4 \mathrm{~Hz}), 7.63(\mathrm{t}, 1 \mathrm{H}, J=8.0 \mathrm{~Hz}), 7.54-7.35$ (m, 4H), 7.09 $(\mathrm{d}, 1 \mathrm{H}), 2.63(\mathrm{~s}, 3 \mathrm{H})$

\section{2-(4-Methoxyphenyl)-6-methyl-pyridine (7b)}<smiles>COc1ccc(-c2cccc(C)n2)cc1</smiles>

Spectra match literature: Deng, D., Hu, B., Yang, M., and Chen, D., Organometallics 2018, 37, 23862394

Physical description: white solid

${ }^{1}$ H NMR (400 MHz, CDCl$)$ ) 7.93-7.97 (2H, m), 7.59 (1H, t, $\left.J=7.8 \mathrm{~Hz}\right), 7.45(1 \mathrm{H}, \mathrm{d}, J=7.8 \mathrm{~Hz}), 7.03$ $(1 \mathrm{H}, \mathrm{d}, J=7.5 \mathrm{~Hz}), 6.97-7.01(2 \mathrm{H}, \mathrm{m}), 3.86(3 \mathrm{H}, \mathrm{s}), 2.61(3 \mathrm{H}, \mathrm{s})$.

\section{2-Methyl-6-(m-tolyl)pyridine (7c)}<smiles>Cc1cccc(-c2cccc(C)n2)c1</smiles>

Spectra match literature: Yao, X.; Qi, L.; Li, R.; Zhen, Q.; Liu, J.; Zhao, Z.; Shao, Y.; Hu, M.; Chen, J., ACS Comb. Sci. 2020, 22, 114-119. 
Physical description: white solid

${ }^{1}$ H NMR (400 MHz, CDCl $) \delta 7.86(1 \mathrm{H}, \mathrm{s}), 7.78(1 \mathrm{H}, \mathrm{d}, J=7.8 \mathrm{~Hz}), 7.61(1 \mathrm{H}, \mathrm{t}, J=7.7 \mathrm{~Hz}), 7.51(1 \mathrm{H}$, $\mathrm{d}, J=7.8 \mathrm{~Hz}), 7.37(1 \mathrm{H}, \mathrm{t}, J=7.6 \mathrm{~Hz}), 7.23(1 \mathrm{H}, \mathrm{d}, J=7.6 \mathrm{~Hz}), 7.08(1 \mathrm{H}, \mathrm{d}, J=7.6 \mathrm{~Hz}), 2.65(3 \mathrm{H}, \mathrm{s})$, $2.46(3 \mathrm{H}, \mathrm{s})$.

${ }^{13} \mathbf{C}\left\{{ }^{1} \mathbf{H}\right\}$ NMR (101 MHz, $\left.\mathbf{C D C l}_{3}\right) \delta 158.3,157.2,139.8,138.3,136.8,129.5,128.6,127.8,124.2,121.5$, $117.7,24.8,21.6$.

2-Methyl-6-(4-(trifluoromethyl)phenyl)pyridine (7d)<smiles>Cc1cccc(-c2ccc(C(F)(F)F)cc2)n1</smiles>

Spectra match literature: Ackermann, L., Potukuchi, H. K., and Kapdi, A. R., Schulze, C., Chem. Eur. J. 2010, 16, 3300-3303

Physical description: white solid

${ }^{1} \mathbf{H}$ NMR (400 MHz, CDCl $) \delta 8.10(2 \mathrm{H}, \mathrm{d}, J=8.1 \mathrm{~Hz}), 7.71(2 \mathrm{H}, \mathrm{d}, J=8.2 \mathrm{~Hz}), 7.68(1 \mathrm{H}, \mathrm{t}, J=7.7$ $\mathrm{Hz}), 7.55(1 \mathrm{H}, \mathrm{d}, J=7.8 \mathrm{~Hz}), 7.16(1 \mathrm{H}, \mathrm{d}, J=7.6 \mathrm{~Hz}), 2.64(3 \mathrm{H}, \mathrm{s})$.

${ }^{19}$ F NMR (376 MHz, $\left.\mathrm{CDCl}_{3}\right) \delta-64.0$

\section{2-(4-Chlorophenyl)-6-methylpyridine (7e)}<smiles>Cc1cccc(-c2ccc(Cl)cc2)n1</smiles>

Spectra match literature: Ackermann, L., Potukuchi, H. K., Kapdi, A. R., and Schulze, C., Chem. Eur. J. 2010, 16, 3300-3303

Physical description: white solid

${ }^{1}$ H NMR (400 MHz, CDCl $) \delta 7.94(2 \mathrm{H}, \mathrm{d}, J=8.5 \mathrm{~Hz}), 7.63(1 \mathrm{H}, \mathrm{t}, J=7.7 \mathrm{~Hz}), 7.48(1 \mathrm{H}, \mathrm{d}, J=7.8$ $\mathrm{Hz}), 7.43(2 \mathrm{H}, \mathrm{d}, J=8.6 \mathrm{~Hz}), 7.10(1 \mathrm{H}, \mathrm{d}, J=7.6 \mathrm{~Hz}), 2.62(3 \mathrm{H}, \mathrm{s})$

2-Methyl-6-(naphthalen-2-yl)pyridine (7f)<smiles>Cc1cccc(-c2ccc3ccccc3c2)n1</smiles> 
Spectra match literature: Addla, D. and Kantevari, S., Journal of Heterocyclic Chemistry, 2014, 51, E384-E388

Physical description: white solid

${ }^{1}$ H NMR (400 MHz, CDCl$) ~ \delta 8.47(1 \mathrm{H}, \mathrm{s}), 8.14(1 \mathrm{H}, \mathrm{dd}, J=8.6 \mathrm{~Hz}, J=1.8 \mathrm{~Hz}), 7.91-7.97$ (2H, m), 7.84-7.89 (1H, m), 7.65-7.71 (2H, m), 7.47-7.53 (2H, m), 7.11-7.15 (1H, m), $2.68(3 \mathrm{H}, \mathrm{s})$

2-(m-Tolyl)pyridine (9b)<smiles>Cc1cccc(-c2ccccn2)c1</smiles>

Spectra match literature: Rao, X., Liu, C., Qiu, J., and Jin, Z., Org. Biomol. Chem., 2012, 10, 7875-7883 Physical description: white solid

${ }^{1} \mathrm{H}$ NMR (400 MHz, CDCl$) \delta 8.69(1 \mathrm{H}, \mathrm{d}, J=4.8 \mathrm{~Hz}), 7.84(1 \mathrm{H}, \mathrm{s}), 7.71-7.76(3 \mathrm{H}, \mathrm{m}), 7.36(1 \mathrm{H}, \mathrm{t}, J=$ 7.6 Hz), 7.21-7.26 (2H, m), $2.44(3 \mathrm{H}, \mathrm{s})$

\section{2-(4-(Trifluoromethyl)phenyl)pyridine (9c)}<smiles>FC(F)(F)c1ccc(-c2ccccn2)cc1</smiles>

Spectra match literature: Zhang, E., Tang, J., Li, S., Wu, P., Moses, J. E., and Sharpless, K. B., Chem. Eur. J. 2016, 22, 5692-5697

Physical description: white solid

${ }^{1}$ H NMR (300 MHz, CDCl $) \delta 8.73(1 \mathrm{H}, \mathrm{d}, J=3.5 \mathrm{~Hz}), 8.12(2 \mathrm{H}, \mathrm{d}, J=8.2 \mathrm{~Hz}), 7.66-7.86(4 \mathrm{H}, \mathrm{m})$, 7.27-7.33 (1H, m).

\section{2-(3,5-Bis(trifluoromethyl)phenyl)pyridine (9d)}<smiles>FC(F)(F)c1cc(-c2ccccn2)cc(C(F)(F)F)c1</smiles>

Spectra match literature: Xu, M., Zhou, R., Yang, G., Xiao, Q., and Du, W., Inorganica Chimica Acta 2008, 361, 2407-2412 
Physical description: white solid

${ }^{1}$ H NMR (400 MHz, CDCl $) \delta 8.76(1 \mathrm{H}, \mathrm{d}, J=4.7 \mathrm{~Hz}), 8.49(2 \mathrm{H}, \mathrm{s}), 7.92(1 \mathrm{H}, \mathrm{s}), 7.81-7.87(2 \mathrm{H}, \mathrm{m})$, $7.36(1 \mathrm{H}, \mathrm{ddd}, J=6.7,4.9,1.7 \mathrm{~Hz}) \mathrm{ppm}$.

${ }^{19}$ F NMR (376 MHz, $\left.\mathbf{C D C l}_{3}\right) \delta-63.8 \mathrm{ppm}$.

\section{Reduction of Pyridines}

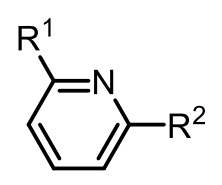

$$
\begin{aligned}
& \text { 1-H }(10 \text { mol\%) } \\
& {\left[\mathrm{Ph}_{3} \mathrm{C}\right]\left[\mathrm{B}\left(\mathrm{C}_{6} \mathrm{~F}_{5}\right)_{4}\right](10 \mathrm{~mol} \%)} \\
& \hline \mathrm{R}_{3} \mathrm{SiH}(1.5 \text { equiv }) \\
& \mathrm{PhMe}, 40 \text { or } 100{ }^{\circ} \mathrm{C}, 19 \mathrm{~h} \\
& \mathrm{H}_{2} \text { (50 or } 90 \text { bar) }
\end{aligned}
$$
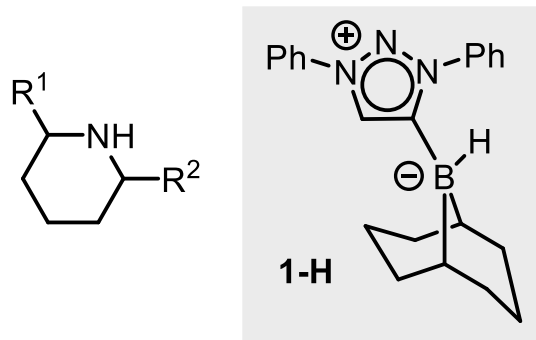

\section{General procedure for the synthesis of pyridine substrates:}

In a glovebox, a 0.5 dram vial was charged with $\left[\mathrm{Ph}_{3} \mathrm{C}\right]\left[\mathrm{B}\left(\mathrm{C}_{6} \mathrm{~F}_{5}\right)_{4}(0.1\right.$ eq.) and $\mathbf{1 - H}(0.1$ eq. $)$ then toluene $(0.5 \mathrm{M})$ was added. The mixture was swirled until the colour changed from red to pale yellow. A second 0.5 dram vial was charged with the pyridine (1.0 eq.) substrate and a magnetic stir bar. The liquid solution in the first vial was directly transferred to the second vial and swirled until dissolved. The hydrosilane (1.5 eq.) was added via an Eppendorf pipette to the vial. The reaction mixture was placed in a $50 \mathrm{~mL}$ stainless steel Parr pressure vessel and sealed with the gas regulator outside the glovebox. The pressure vessel was pressurized and depressurized five times with hydrogen gas (28 bar) then brought up to the required pressure before heating ( 45 bar for $40{ }^{\circ} \mathrm{C}, 76$ bar for $100{ }^{\circ} \mathrm{C}$ ). The vessel was then submerged in an oil bath of the proper temperature and heated for $19 \mathrm{~h}$. Afterwards, the vessel was slowly depressurized. Internal standard (ferrocene) was added to the reaction mixture to enable analysis of the crude by NMR spectroscopy. Then the crude reaction mixture was added to $10 \mathrm{~mL}$ of distilled $\mathrm{H}_{2} \mathrm{O}$ and the aqueous layer was extracted three times with ethyl acetate. The organic fractions were dried over $\mathrm{Na}_{2} \mathrm{SO}_{4}$, filtered and evaporated. After drying, the resulting oil was subjected to silica gel column chromatography to give the desired product as either an oil or solid.

\section{2-Methyl-6-phenylpiperidine (8a)}<smiles>CC1CCCC(c2ccccc2)N1</smiles> 
Prepared from pyridine $7 \mathbf{a}(21.2 \mathrm{mg}, 0.125 \mathrm{mmol})$, phenylsilane $(23.1 \mathrm{~mL}, 0.19 \mathrm{mmol}),\left[\mathrm{Ph}_{3} \mathrm{C}\right]\left[\mathrm{B}\left(\mathrm{C}_{6} \mathrm{~F}_{5}\right)_{4}\right]$ (11.5 mg, $0.013 \mathrm{mmol})$ and $\mathbf{1}-\mathbf{H}(4.4 \mathrm{mg}, 0.013 \mathrm{mmol}$.) in toluene $(0.25 \mathrm{~mL})$ according to General

Procedure. The crude residue was purified by column chromatography to give 8a (colourless oil, $19 \mathrm{mg}$, $87 \%)$.

Spectra match literature: Liu, Y., Du, H. J. Am. Chem. Soc. 2013, 135, 12968-12971.

${ }^{1}$ H NMR (700 MHz, CDCl$) \delta 7.37(2 \mathrm{H}, \mathrm{d}, J=7.3 \mathrm{~Hz}), 7.31(2 \mathrm{H}, \mathrm{t}, J=7.5 \mathrm{~Hz}), 7.23(1 \mathrm{H}, \mathrm{t}, J=7.4 \mathrm{~Hz})$, $3.66(1 \mathrm{H}, \mathrm{dd}, J=11.1,2.5 \mathrm{~Hz}), 2.81(1 \mathrm{H}, \mathrm{m}), 1.88(1 \mathrm{H}, \mathrm{dt}, J=12.9,3.1 \mathrm{~Hz}), 1.76(1 \mathrm{H}, \mathrm{m}), 1.65(1 \mathrm{H}, \mathrm{m})$, $1.51(1 \mathrm{H}, \mathrm{qt}, J=13.0,3.8 \mathrm{~Hz}), 1.45(1 \mathrm{H}, \mathrm{qd}, J=12.9,3.6 \mathrm{~Hz}), 1.09-1.20(5 \mathrm{H}, \mathrm{m}) \mathrm{ppm}$.

${ }^{13} \mathbf{C}\left\{{ }^{1} \mathbf{H}\right\}$ NMR (176 MHz, $\left.\mathbf{C D C l}_{3}\right) \delta 145.7,128.5,127.1,126.9,62.6,53.4,34.4,34.0,25.5,23.2 \mathrm{ppm}$.

\section{2-(4-Methoxyphenyl)-6-methylpiperidine (8b)}<smiles>COc1ccc(C2CCCC(C)N2)cc1</smiles>

Prepared from pyridine $7 \mathbf{b}(49.8 \mathrm{mg}, 0.25 \mathrm{mmol})$, phenylsilane $(46.2 \mathrm{~mL}, 0.38 \mathrm{mmol}),\left[\mathrm{Ph}_{3} \mathrm{C}\right]\left[\mathrm{B}\left(\mathrm{C}_{6} \mathrm{~F}_{5}\right)_{4}\right]$ $(23.1 \mathrm{mg}, 0.025 \mathrm{mmol})$ and $\mathbf{1}-\mathbf{H}(10.3 \mathrm{mg}, 0.03 \mathrm{mmol})$ in toluene $(0.5 \mathrm{~mL})$ according to General Procedure. The crude residue was purified by column chromatography to give $\mathbf{8 b}$ (colourless oil, $26.5 \mathrm{mg}, 52 \%$ ).

Spectra match literature: Liu, Y., Du, H. J. Am. Chem. Soc. 2013, 135, 12968-12971.

${ }^{1}$ H NMR (700 MHz, CDCl $) \delta 7.29(2 \mathrm{H}, \mathrm{d}, J=8.5 \mathrm{~Hz}), 6.85(2 \mathrm{H}, \mathrm{d}, J=8.7 \mathrm{~Hz}), 3.79(3 \mathrm{H}, \mathrm{s}), 3.60(1 \mathrm{H}$, dd, $J=10.9,2.7$ Hz), 2.76-2.83 (1H, m), 1.81-1.90 (1H, m), 1.61-1.75 (3H, m), 1.38-1.6 (2H, m), 1.09$1.18(4 \mathrm{H}, \mathrm{m}) \mathrm{ppm}$.

${ }^{13}$ C NMR (176 MHz, $\left.\mathbf{C D C l}_{3}\right) \delta 158.7,138.0,127.9,113.8,62.0,55.4,53.4,34.4,34.0,25.5,23.2$ ppm.

\section{2-Methyl-6-(m-tolyl)piperidine (8c)}<smiles>Cc1cccc(C2CCCC(C)N2)c1</smiles>

Prepared from pyridine $7 \mathrm{c}(22.9 \mathrm{mg}, 0.125 \mathrm{mmol})$, phenylsilane $(23.1 \mathrm{~mL}, 0.19 \mathrm{mmol}),\left[\mathrm{Ph}_{3} \mathrm{C}\right]\left[\mathrm{B}\left(\mathrm{C}_{6} \mathrm{~F}_{5}\right)_{4}\right]$ $(11.5 \mathrm{mg}, 0.013 \mathrm{mmol})$ and $\mathbf{1}-\mathbf{H}(5.1 \mathrm{mg}, 0.015 \mathrm{mmol}$.) in toluene $(0.25 \mathrm{~mL})$ according to General Procedure. The crude residue was purified by column chromatography to give $\mathbf{8 c}$ (colourless oil, $18 \mathrm{mg}$, $76 \%)$.

${ }^{1}$ H NMR (700 MHz, CDCl $) \delta 7.22(1 \mathrm{H}, \mathrm{s}), 7.20(1 \mathrm{H}, \mathrm{t}, J=7.5 \mathrm{~Hz}), 7.16(1 \mathrm{H}, \mathrm{d}, J=7.6 \mathrm{~Hz}), 7.05(1 \mathrm{H}$, $\mathrm{d}, J=7.4 \mathrm{~Hz}), 3.63(1 \mathrm{H}, \mathrm{dd}, J=10.9,2.1 \mathrm{~Hz}), 2.81(1 \mathrm{H}, \mathrm{m}), 2.35(3 \mathrm{H}, \mathrm{s}), 1.88(1 \mathrm{H}, \mathrm{m}), 1.76(1 \mathrm{H}, \mathrm{d}, J=$ 
$12.0 \mathrm{~Hz}), 1.65(1 \mathrm{H}, \mathrm{dd}, J=10.8,1.7 \mathrm{~Hz}), 1.58(1 \mathrm{H}$, br. s), 1.43-1.54 (2H, m), $1.16(1 \mathrm{H}, \mathrm{qd}, J=12.7,3.7$ $\mathrm{Hz}), 1.12(3 \mathrm{H}, \mathrm{d}, J=6.2 \mathrm{~Hz}) \mathrm{ppm}$.

${ }^{13} \mathbf{C}\left\{{ }^{1} \mathbf{H}\right\}$ NMR (176 MHz, $\left.\mathbf{C D C l}_{3}\right) \delta 145.6,138.1,128.4,127.8,127.4,124.0,62.7,53.4,34.4,34.0,25.6$, 23.2, $21.6 \mathrm{ppm}$.

HRMS (EI): calcd. for $\mathrm{C}_{13} \mathrm{H}_{19} \mathrm{~N} 189.1517$, found 189.1508

\section{2-Methyl-6-(4-(trifluoromethyl)phenyl)piperidine (8d)}<smiles>CC1CCCC(c2ccc(C(F)(F)F)cc2)N1</smiles>

Prepared from pyridine $7 \mathbf{d}(29.6 \mathrm{mg}, 0.125 \mathrm{mmol})$, phenylsilane $(23.1 \mathrm{~mL}, 0.19 \mathrm{mmol}),\left[\mathrm{Ph}_{3} \mathrm{C}\right]\left[\mathrm{B}\left(\mathrm{C}_{6} \mathrm{~F}_{5}\right)_{4}\right]$ (11.5 mg, $0.013 \mathrm{mmol})$ and $\mathbf{1 - H}\left(5.1 \mathrm{mg}, 0.015 \mathrm{mmol}\right.$.) in toluene $(0.25 \mathrm{~mL})$ at $90{ }^{\circ} \mathrm{C}$ according to General Procedure. The crude residue was purified by column chromatography to give $\mathbf{8 d}$ (colourless oil, $19.4 \mathrm{mg}$, $64 \%)$.

Spectra match literature: Liu, Y., Du, H. J. Am. Chem. Soc. 2013, 135, 12968-12971.

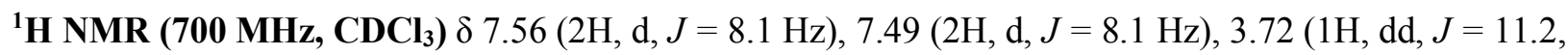
$2.7 \mathrm{~Hz}), 2.79-2.84(1 \mathrm{H}, \mathrm{m}), 1.86-1.90(1 \mathrm{H}, \mathrm{m}), 1.75(1 \mathrm{H}, \mathrm{dd}, J=12.3,2.6 \mathrm{~Hz}), 1.66(1 \mathrm{H}, \mathrm{dd}, J=15.4$, $2.5 \mathrm{~Hz}), 1.59(1 \mathrm{H}$, br. s), $1.51(1 \mathrm{H}, \mathrm{qt}, J=13.1,3.9 \mathrm{~Hz}), 1.37-1.43(1 \mathrm{H}, \mathrm{m}), 1.13-1.18(1 \mathrm{H}, \mathrm{m}), 1.12(3 \mathrm{H}$, $\mathrm{d}, J=6.2 \mathrm{~Hz}) \mathrm{ppm}$.

${ }^{13}$ C NMR (176 MHz, $\left.\mathbf{C D C l}_{3}\right) \delta 149.8,129.3$ (q, $\left.J=32 \mathrm{~Hz}\right), 127.2,125.4(\mathrm{q}, J=4 \mathrm{~Hz}), 124.4(\mathrm{q}, J=272$ $\mathrm{Hz}), 62.2,53.2,34.6,33.8,25.4,23.2 \mathrm{ppm}$.

${ }^{19}$ F NMR (376 MHz, CDCl$\left._{3}\right) \delta-63.8$ ppm.

\section{2-(4-Chlorophenyl)-6-methylpiperidine (8e)}<smiles>CC1CCCC(c2ccc(Cl)cc2)N1</smiles>

Prepared from pyridine 7 e $(50.9 \mathrm{mg}, 0.25 \mathrm{mmol})$, phenylsilane $(46.2 \mathrm{~mL}, 0.38 \mathrm{mmol}),\left[\mathrm{Ph}_{3} \mathrm{C}\right]\left[\mathrm{B}\left(\mathrm{C}_{6} \mathrm{~F}_{5}\right)_{4}\right]$ (23.1 mg, $0.025 \mathrm{mmol})$ and $\mathbf{1 - H}\left(10.3 \mathrm{mg}, 0.03 \mathrm{mmol}\right.$.) in toluene $(0.5 \mathrm{~mL})$ at $90{ }^{\circ} \mathrm{C}$ according to General Procedure. The crude residue was purified by column chromatography to give $\mathbf{8 e}$ (colourless oil, $32.5 \mathrm{mg}$, $62 \%)$.

Spectra match literature: Liu, Y., Du, H. J. Am. Chem. Soc. 2013, 135, 12968-12971. 
${ }^{1}$ H NMR (400 MHz, CDCl $) \delta 7.31(2 \mathrm{H}, \mathrm{d}, J=8.6 \mathrm{~Hz}), 7.26(2 \mathrm{H}, \mathrm{d}, J=8.6 \mathrm{~Hz}), 3.63(1 \mathrm{H}, \mathrm{dd}, J=11.1$, $2.7 \mathrm{~Hz}), 2.75-2.83(1 \mathrm{H}, \mathrm{m}), 1.83-1.90(1 \mathrm{H}, \mathrm{m}), 1.72(1 \mathrm{H}, \mathrm{dd}, J=12.1,2.0 \mathrm{~Hz}), 1.64(1 \mathrm{H}, \mathrm{dd}, J=12.5$, $2.2 \mathrm{~Hz}), 1.32-1.56(3 \mathrm{H}, \mathrm{m}), 1.08-1.22(4 \mathrm{H}, \mathrm{m}) \mathrm{ppm}$.

${ }^{13} \mathbf{C}$ NMR $\left\{{ }^{1} \mathbf{H}\right\}\left(\mathbf{1 0 1} \mathbf{M H z}, \mathbf{C D C l}_{3}\right) \delta 144.3,132.6,128.6,128.2,61.9,53.2,34.6,33.9,25.4,23.2 \mathrm{ppm}$.

\section{2-Methyl-6-(naphthalen-2-yl)piperidine (8f)}<smiles>CC1CCCC(c2ccc3ccccc3c2)N1</smiles>

Prepared from pyridine $7 \mathbf{f}(27.4 \mathrm{mg}, 0.125 \mathrm{mmol})$, phenylsilane $(23.1 \mathrm{~mL}, 0.19 \mathrm{mmol}),\left[\mathrm{Ph}_{3} \mathrm{C}\right]\left[\mathrm{B}\left(\mathrm{C}_{6} \mathrm{~F}_{5}\right)_{4}\right]$ (11.5 mg, $0.013 \mathrm{mmol})$ and $\mathbf{1 - H}\left(5.1 \mathrm{mg}, 0.015 \mathrm{mmol}\right.$.) in toluene $(0.25 \mathrm{~mL})$ at $90{ }^{\circ} \mathrm{C}$ according to General Procedure. The crude residue was purified by column chromatography to give $\mathbf{8 f}$ (colourless oil, $22.3 \mathrm{mg}$, $79 \%)$.

Spectra match literature: Liu, Y., Du, H. J. Am. Chem. Soc. 2013, 135, 12968-12971.

${ }^{1}$ H NMR (700 MHz, CDCl$)$ ) 7.79-7.85 (4H, m), 7.52 (1H, d, $J=8.5$ Hz), 7.42-7.47 (2H, m), $3.83(1 \mathrm{H}$, dd, $J=10.8,2.7 \mathrm{~Hz}), 2.85-2.90(1 \mathrm{H}, \mathrm{m}), 1.91-1.95(1 \mathrm{H}, \mathrm{m}), 1.86(1 \mathrm{H}, \mathrm{d}, J=11.6), 1.74$ (1H, br. s), 1.70 $(1 \mathrm{H}, \mathrm{dd}, J=12.1,2.4 \mathrm{~Hz}), 1.50-1.61(2 \mathrm{H}, \mathrm{m}), 1.19-1.27(1 \mathrm{H}, \mathrm{m}), 1.17(3 \mathrm{H}, \mathrm{dd}, J=6.3,1.3 \mathrm{~Hz}) \mathrm{ppm}$. ${ }^{13}$ C NMR (176 MHz, $\mathbf{C D C l}_{3}$ ) $\delta$ 143.2, 133.7, 132.9, 128.0, 128.0, 127.7, 126.0, 125.7, 125.6, 124.9, 62.7, $53.4,34.5,34.0,25.6,23.2 \mathrm{ppm}$.<smiles>CC1CCCC(C)N1</smiles>

Prepared from pyridine $7 \mathbf{g}(28.3 \mathrm{mg}, 0.25 \mathrm{mmol})$, phenylsilane $(46.2 \mathrm{~mL}, 0.38 \mathrm{mmol}),\left[\mathrm{Ph}_{3} \mathrm{C}\right]\left[\mathrm{B}\left(\mathrm{C}_{6} \mathrm{~F}_{5}\right)_{4}\right]$ (23.1 mg, $0.025 \mathrm{mmol})$ and $\mathbf{1 - H}\left(10.3 \mathrm{mg}, 0.03 \mathrm{mmol}\right.$.) in toluene $(2 \mathrm{~mL})$ at $40{ }^{\circ} \mathrm{C}$ according to General Procedure. 1,3,5-Trimethoxy benzene $(9.2 \mathrm{mg}, 0.055 \mathrm{mmol})$ was added to the resulting reaction mixture as an internal standard, and the crude residue was analyzed by ${ }^{1} \mathrm{H}$ NMR, which shows $47 \%$ of $\mathbf{8 g}$ was obtained with $50 \%$ of unreacted $7 \mathrm{~g}$.

\section{2-Phenylpiperidine (10a)}<smiles>c1ccc(C2CCCCN2)cc1</smiles> 
Prepared from pyridine 9a $(0.14 \mathrm{~mL}, 1 \mathrm{mmol})$, phenyldimethylsilane $(0.23 \mathrm{~mL}, 1.5 \mathrm{mmol})$, $\left[\mathrm{Ph}_{3} \mathrm{C}\right]\left[\mathrm{B}\left(\mathrm{C}_{6} \mathrm{~F}_{5}\right)_{4}\right](92 \mathrm{mg}, 0.1 \mathrm{mmol})$ and $\mathbf{1 - H}(35 \mathrm{mg}, 0.1 \mathrm{mmol}$.) in toluene $(2 \mathrm{~mL})$ according to General Procedure. The crude residue was purified by preparative thin layer chromatography to give 10a (colourless oil, $143 \mathrm{mg}, 89 \%$ ).

Spectra match literature: Yamaguchi, R., Kawagoe, S., Asai, C., and Fujita, K., Org. Lett. 2008, 10, 181184.

${ }^{1}$ H NMR (400 MHz, CDCl $) \delta 7.36(2 \mathrm{H}, \mathrm{d}, J=7.1 \mathrm{~Hz}), 7.31(2 \mathrm{H}, \mathrm{t}, J=7.2 \mathrm{~Hz}), 7.22(1 \mathrm{H}, \mathrm{t}, J=7.2$ $\mathrm{Hz}), 3.60(1 \mathrm{H}, \mathrm{dd}, J=10.0,2.2 \mathrm{~Hz}), 3.18(1 \mathrm{H}, \mathrm{d}, J=11.2 \mathrm{~Hz}), 2.80(1 \mathrm{H}, \mathrm{td}, J=11.4,2.7 \mathrm{~Hz}), 1.85-1.92$ $(1 \mathrm{H}, \mathrm{m}), 1.73-1.84(2 \mathrm{H}, \mathrm{m}), 1.62-1.71(1 \mathrm{H}, \mathrm{m}), 1.42-1.61(3 \mathrm{H}, \mathrm{m}) \mathrm{ppm}$.

${ }^{13}$ C NMR (176 MHz, CDCl $) \delta$ 145.0, 128.5, 127.3, 126.8, 62.4, 47.7, 34.7, 25.7, 25.4 ppm.

\section{2-(m-Tolyl)piperidine (10b)}<smiles>Cc1cccc(C2CCCCN2)c1</smiles>

Prepared from pyridine 9b $(42.3 \mathrm{mg}, 0.25 \mathrm{mmol})$, phenyldimethylsilane $(57.6 \mu \mathrm{L}, 0.38 \mathrm{mmol})$, $\left[\mathrm{Ph}_{3} \mathrm{C}\right]\left[\mathrm{B}\left(\mathrm{C}_{6} \mathrm{~F}_{5}\right)_{4}\right](23.1 \mathrm{mg}, 0.025 \mathrm{mmol})$ and $\mathbf{1 - H}(10.3 \mathrm{mg}, 0.03 \mathrm{mmol}$.) in toluene $(0.5 \mathrm{~mL})$ according to General Procedure. The crude residue was purified by column chromatography to give $\mathbf{1 0 b}$ (colourless oil, $35.2 \mathrm{mg}, 81 \%)$.

${ }^{1}$ H NMR (700 MHz, CDCl $)$ ) 7.19-7.21 (2H, m), $7.14(1 \mathrm{H}, \mathrm{d}, J=4.5 \mathrm{~Hz}), 7.05(1 \mathrm{H}, \mathrm{d}, J=4.0 \mathrm{~Hz})$, $3.56(1 \mathrm{H}, \mathrm{dd}, J=6.0,1.5 \mathrm{~Hz}), 3.18-3.21(1 \mathrm{H}, \mathrm{m}), 2.79(1 \mathrm{H}, \mathrm{td}, J=6.7,1.5 \mathrm{~Hz}), 2.34(3 \mathrm{H}, \mathrm{s}), 1.94(1 \mathrm{H}$, br. s), $1.87-1.90(1 \mathrm{H}, \mathrm{m}), 1.78(1 \mathrm{H}, \mathrm{m}), 1.66(1 \mathrm{H}, \mathrm{m}), 1.45-1.58(3 \mathrm{H}, \mathrm{m}) \mathrm{ppm}$.

${ }^{13}$ C NMR (176 MHz, $\left.\mathbf{C D C l}_{3}\right) \delta 145.6,138.1,128.4,127.9,127.4,123.8,62.5,48.0,35.1,26.0,25.6$, $21.5 \mathrm{ppm}$.

HRMS (EI): calcd. for $\mathrm{C}_{12} \mathrm{H}_{17} \mathrm{~N} 175.1361$, found 175.1369

\section{2-(4-(Trifluoromethyl)phenyl)piperidine (10c)}<smiles>FC(F)(F)c1ccc(C2CCCCN2)cc1</smiles>

Prepared from pyridine 9c $(55.8 \mathrm{mg}, 0.25 \mathrm{mmol})$, phenyldimethylsilane $(57.6 \mu \mathrm{L}, 0.38 \mathrm{mmol})$, $\left[\mathrm{Ph}_{3} \mathrm{C}\right]\left[\mathrm{B}\left(\mathrm{C}_{6} \mathrm{~F}_{5}\right)_{4}\right](23.1 \mathrm{mg}, 0.025 \mathrm{mmol})$ and $\mathbf{1 - H}(10.3 \mathrm{mg}, 0.03 \mathrm{mmol}$.) in toluene $(0.5 \mathrm{~mL})$ according to 
General Procedure. The crude residue was purified by column chromatography to give 10c (colourless oil, $31.5 \mathrm{mg}, 57 \%)$.

Spectra match literature: Martins, J. E. D., Redondo, M. A. C., and Wills, M., Tetrahedron: Asymmetry 2010, 21, 2258.

${ }^{1}$ H NMR (700 MHz, CDCl $) \delta 7.56(2 \mathrm{H}, \mathrm{d}, J=8.1 \mathrm{~Hz}), 7.48(2 \mathrm{H}, \mathrm{d}, J=8.0 \mathrm{~Hz}), 3.66(1 \mathrm{H}, \mathrm{d}, J=10.6$ $\mathrm{Hz}), 3.21(1 \mathrm{H}, \mathrm{d}, J=11.7 \mathrm{~Hz}), 2.80(1 \mathrm{H}, \mathrm{td}, J=11.5,2.2 \mathrm{~Hz}), 1.90(1 \mathrm{H}, \mathrm{d}, J=10.2 \mathrm{~Hz}), 1.78(1 \mathrm{H}, \mathrm{d}, J=$ $11.3 \mathrm{~Hz}), 1.67(1 \mathrm{H}, \mathrm{d}, J=12.4 \mathrm{~Hz}), 1.44-1.57(3 \mathrm{H}, \mathrm{m}) \mathrm{ppm}$.

${ }^{13}$ C NMR (176 MHz, $\left.\mathbf{C D C l}_{3}\right) \delta$ 149.8, 129.4 (q, $\left.J=32.4 \mathrm{~Hz}\right), 127.1,125.4$ (q, $\left.J=3.8 \mathrm{~Hz}\right), 124.4(\mathrm{q}, J=$ $271.8 \mathrm{~Hz}), 62.1,47.8,35.3,25.9,25.4 \mathrm{ppm}$.

\section{2-(3,5-Bis(trifluoromethyl)phenyl)pyridine (10d)}<smiles>FC(F)(F)c1cc(C2CCCCN2)cc(C(F)(F)F)c1</smiles>

Prepared from pyridine 9d (36.4 mg, $0.125 \mathrm{mmol})$, phenyldimethylsilane $(28.8 \mu \mathrm{L}, 0.19 \mathrm{mmol})$, $\left[\mathrm{Ph}_{3} \mathrm{C}\right]\left[\mathrm{B}\left(\mathrm{C}_{6} \mathrm{~F}_{5}\right)_{4}\right](11.5 \mathrm{mg}, 0.013 \mathrm{mmol})$ and $\mathbf{1 - H}(5.1 \mathrm{mg}, 0.015 \mathrm{mmol}$.) in toluene $(0.25 \mathrm{~mL})$ according to General Procedure. The crude residue was purified by column chromatography to give 10d (colourless oil, $18 \mathrm{mg}, 48 \%$ ).

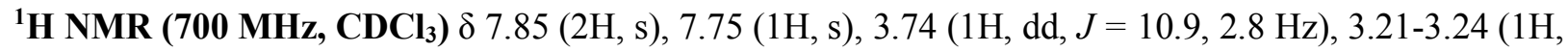
m), $2.81(1 \mathrm{H}, \mathrm{td}, J=11.6,2.6 \mathrm{~Hz}), 1.90-1.93(1 \mathrm{H}, \mathrm{m}), 1.84(1 \mathrm{H}, \mathrm{br} . \mathrm{s}), 1.80-1.83(1 \mathrm{H}, \mathrm{m}), 1.67-1.70(1 \mathrm{H}$, $\mathrm{m}), 1.44-1.58(3 \mathrm{H}, \mathrm{m}) \mathrm{ppm}$.

${ }^{13}$ C NMR (176 MHz, $\left.\mathbf{C D C l}_{3}\right) \delta$ 148.3, 131.7 (q, $\left.J=33 \mathrm{~Hz}\right), 127.1$ (q, $\left.J=3 \mathrm{~Hz}\right), 123.6$ (q, $\left.J=272 \mathrm{~Hz}\right)$, 121.2 (sept, $J=5 \mathrm{~Hz}$ ), 61.6, 47.6, 35.4, 25.7, $25.3 \mathrm{ppm}$.

${ }^{19}$ F NMR (376 MHz, $\left.\mathbf{C D C l}_{3}\right) \delta-63.7 \mathrm{ppm}$.

HRMS (EI): calcd. for $\mathrm{C}_{13} \mathrm{H}_{13} \mathrm{~F}_{6} \mathrm{~N}$ 297.0952, found 297.0959

\section{Large Scale Reduction of 1-Phenyl Pyridine 9a with 2.5 mol\% of Borenium Catalyst}

In a glovebox, a 1 dram vial was charged with $\left[\mathrm{Ph}_{3} \mathrm{C}\right]\left[\mathrm{B}\left(\mathrm{C}_{6} \mathrm{~F}_{5}\right)_{4}\right](73.8 \mathrm{mg}, 0.08 \mathrm{mmol})$ and $\mathbf{1 - H}(30.0 \mathrm{mg}$, $0.09 \mathrm{mmol})$ then toluene $(1.6 \mathrm{~mL})$ was added. The mixture was swirled until the colour changed from red to pale yellow. A second 1 dram vial was charged with 2-phenyl pyridine $(0.5 \mathrm{~g}, 3.2 \mathrm{mmol})$ and dimethylphenylsilane $(0.74 \mathrm{mmL}, 4.8 \mathrm{mmol})$ in toluene $(1.6 \mathrm{~mL})$. These solutions were transferred to a 50 $\mathrm{mL}$ glass tube equipped with a magnetic stir bar. The reaction mixture was placed in a $50 \mathrm{~mL}$ stainless steel 
Parr pressure vessel and sealed with the gas regulator outside the glovebox. The pressure vessel was pressurized and depressurized five times with hydrogen gas (28 bar) then brought up to the required pressure before heating (76 bar). The vessel was then submerged in an oil bath at $100{ }^{\circ} \mathrm{C}$ and heated for $60 \mathrm{~h}$. Afterwards the vessel was slowly depressurized. Internal standard (1,3,5-trimethoxybenzene) was added to the reaction mixture. Then the crude reaction mixture was diluted with $3 \mathrm{~mL}$ of ethyl acetate and added to $10 \mathrm{~mL}$ of distilled $\mathrm{H}_{2} \mathrm{O}$. The aqueous layer was extracted three times with $3 \mathrm{~mL}$ of ethyl acetate. The organic fractions were dried over $\mathrm{Na}_{2} \mathrm{SO}_{4}$, filtered and evaporated. After drying, the resulting oil was subjected to silica gel column chromatography (ethyl acetate:hexane:triethylamine $=1: 15: 1$ ) to give the desired product as a colorless oil along with siloxane by-product. The obtained product was dissolved in $5 \mathrm{~mL}$ of ethyl ether and extracted with $2 \mathrm{M} \mathrm{HCl}$ aq. three times. Then the combined water phase was neutralized with $2 \mathrm{M} \mathrm{NaOH}$ aq. and extracted with ethyl ether three times. The combined organic phase was dried over $\mathrm{Na}_{2} \mathrm{SO}_{4}$, filtered and evaporated to give the desired product $(447 \mathrm{mg}, 2.76 \mathrm{mmol}, 86 \%)$ as a colorless solid. 


\section{Control Experiments}

\section{Catalyst Decomposition}

In a glovebox, a 0.5 dram vial was charged with $\left[\mathrm{Ph}_{3} \mathrm{C}\right]\left[\mathrm{B}\left(\mathrm{C}_{6} \mathrm{~F}_{5}\right)_{4}\right](23.1 \mathrm{mg}, 0.025 \mathrm{mmol})$ and $1-\mathrm{H}(8.6 \mathrm{mg}$, $0.025 \mathrm{mmol}$ ) then $0.5 \mathrm{~mL}$ of 1,2-dichloroethane was added. The mixture was swirled until the colour changed from red to pale yellow. Diisopropylamine ( $35 \mu \mathrm{L}, 0.25 \mathrm{mmol})$ was added to the mixture and the solution was transferred to a J-Young tube. The solution was heated at $90{ }^{\circ} \mathrm{C}$ for $96 \mathrm{~h}$ after which the reaction was monitored by NMR spectroscopy.
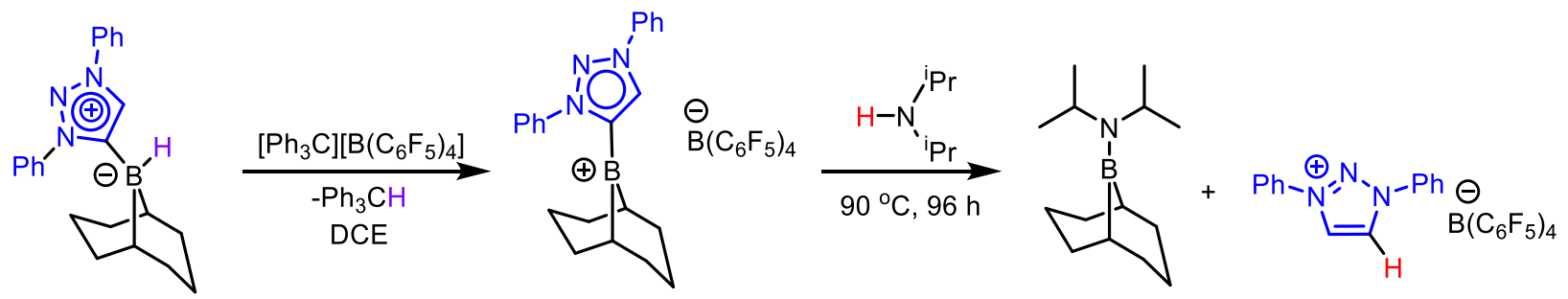


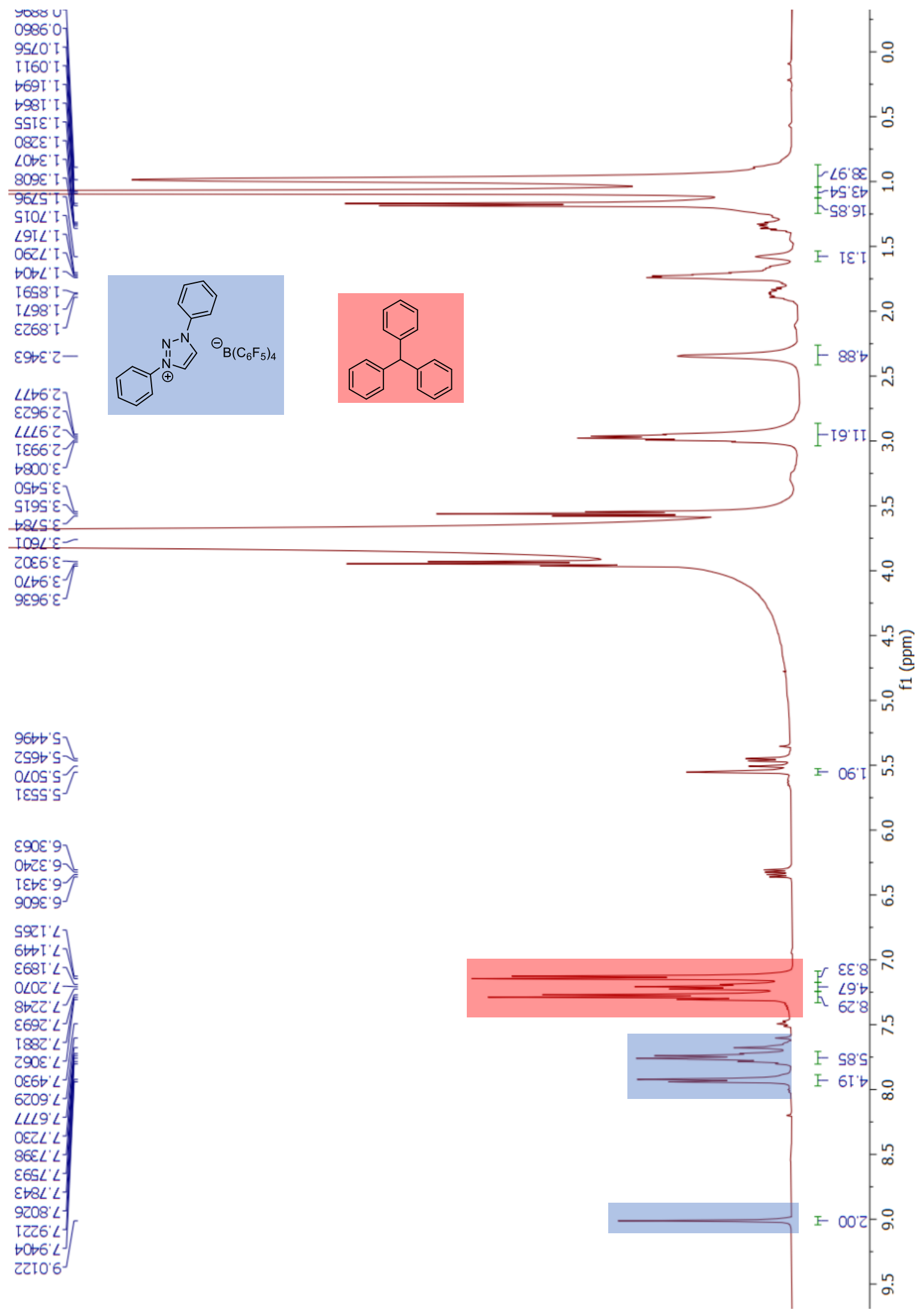

Figure S1. Crude catalyst decomposition reaction, 1H NMR (700 MHz) in 1,2-dichloroethane 

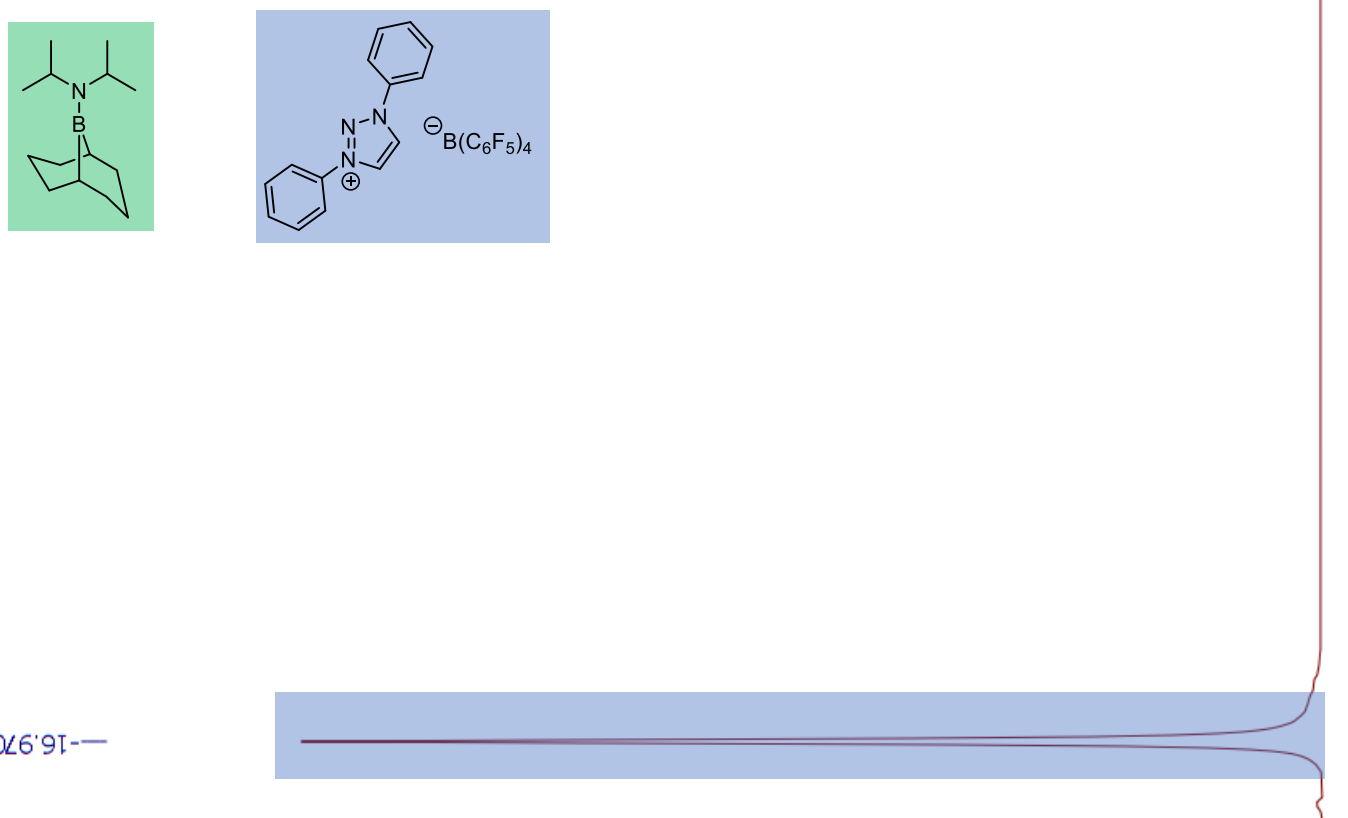


\section{Mechanistic Studies}

\subsection{Control Experiments}

\section{Reduction of 2,6-lutidine without borenium precursor 1-H}

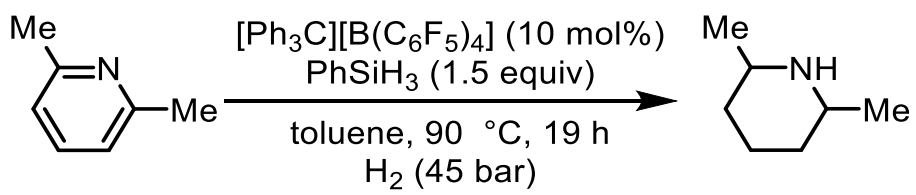

In a glovebox, a 0.5 dram vial was charged with $\left[\mathrm{Ph}_{3} \mathrm{C}\right]\left[\mathrm{B}\left(\mathrm{C}_{6} \mathrm{~F}_{5}\right)_{4}\right](11.5 \mathrm{mg}, 0.0125 \mathrm{mmol})$ and phenylsilane $(23.1 \mathrm{~mL}, 0.19 \mathrm{mmol})$ then $0.5 \mathrm{~mL}$ of toluene was added. The mixture was swirled until the colour changed from red to pale yellow. 2,6-lutidine $(13 \mathrm{mg}, 0.125 \mathrm{mmol}$ ) was added to the solution. The reaction mixture was placed in a $50 \mathrm{~mL}$ stainless steel Parr pressure vessel and sealed with the gas regulator outside the glovebox. The pressure vessel was pressurized and depressurized five times with hydrogen gas (28 bar) then brought up to the required pressure before heating ( 45 bar). The vessel was then submerged in an oil bath of $90^{\circ} \mathrm{C}$ and heated for $19 \mathrm{~h}$. After cooling the reaction to room temperature, the crude mixture was analyzed by ${ }^{1} \mathrm{H}$ NMR and ${ }^{13} \mathrm{C}$ NMR spectroscopy showing no reduced product formation.

\section{Reduction of 2-phenyl pyridine with various changes to reaction conditions}

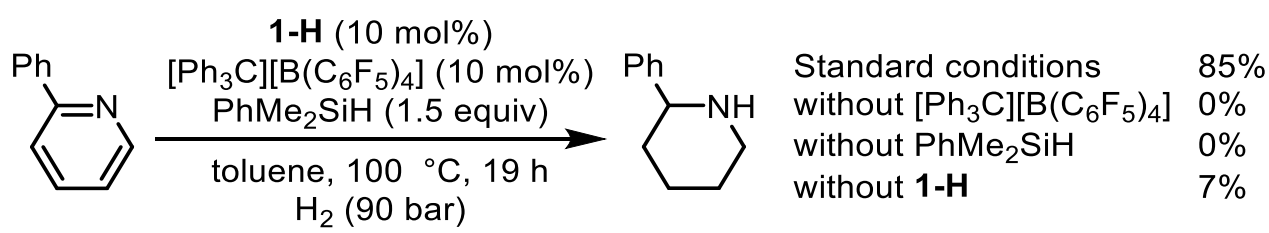

Without $\left[\mathbf{P h}_{3} \mathbf{C}\right]\left[\mathbf{B}\left(\mathbf{C}_{6} \mathbf{F}_{5}\right)_{4}\right]$ : In a glovebox, a 0.5 dram vial was charged with $\mathbf{1 - H}(6.9 \mathrm{mg}, 0.02 \mathrm{mmol})$ then $0.4 \mathrm{~mL}$ of toluene was added. 2-Phenylpyridine (31 $\mathrm{mg}, 0.2 \mathrm{mmol}$ ) was added to the solution followed by phenyldimethylsilane $(41 \mathrm{mg}, 0.3 \mathrm{mmol}$ ) added directly to the vial. The reaction mixture was placed in a $50 \mathrm{~mL}$ stainless steel Parr pressure vessel and sealed with the gas regulator outside the glovebox. The pressure vessel was pressurized and depressurized five times with hydrogen gas (28 bar) then brought up to the required pressure before heating ( 76 bar). The vessel was then submerged in an oil bath of $100{ }^{\circ} \mathrm{C}$ and heated for $19 \mathrm{~h}$. After cooling the reaction to room temperature, the crude mixture was analyzed by ${ }^{1} \mathrm{H}$ NMR spectroscopy showing no reduced product formation.

Without Silane: In a glovebox, a 0.5 dram vial was charged with $\left[\mathrm{Ph}_{3} \mathrm{C}\right]\left[\mathrm{B}\left(\mathrm{C}_{6} \mathrm{~F}_{5}\right)_{4}\right](18 \mathrm{mg}, 0.02 \mathrm{mmol})$ and 1-H (6.9 mg, $0.02 \mathrm{mmol})$ then $0.4 \mathrm{~mL}$ of toluene was added. 2-phenylpyridine (31 $\mathrm{mg}, 0.2 \mathrm{mmol}$ ) was added to the solution directly to the vial. The reaction mixture was placed in a $50 \mathrm{~mL}$ stainless steel Parr 
pressure vessel and sealed with the gas regulator outside the glovebox. The pressure vessel was pressurized and depressurized five times with hydrogen gas ( 28 bar) then brought up to the required pressure before heating (76 bar). The vessel was then submerged in an oil bath of $100^{\circ} \mathrm{C}$ and heated for $19 \mathrm{~h}$. After cooling the reaction to room temperature, the crude mixture was analyzed by ${ }^{1} \mathrm{H}$ NMR spectroscopy showing no reduced product formation.

Without Borenium precursor 1-H: In a glovebox, a 0.5 dram vial was charged with $\left[\mathrm{Ph}_{3} \mathrm{C}\right]\left[\mathrm{B}\left(\mathrm{C}_{6} \mathrm{~F}_{5}\right)_{4}\right](23$ $\mathrm{mg}, 0.025 \mathrm{mmol}$ ), 2-phenylpyridine $(39 \mathrm{mg}, 0.25 \mathrm{mmol})$, and $0.25 \mathrm{~mL}$ of toluene was added. Then, phenyldimethylsilane (51 mg, $0.38 \mathrm{mmol}$ ) in $0.25 \mathrm{~mL}$ of toluene was added to the solution directly to the vial. The reaction mixture was placed in a $50 \mathrm{~mL}$ stainless steel Parr pressure vessel and sealed with the gas regulator outside the glovebox. The pressure vessel was pressurized and depressurized five times with hydrogen gas (28 bar) then brought up to the required pressure before heating ( 76 bar). The vessel was then submerged in an oil bath of $100{ }^{\circ} \mathrm{C}$ and heated for $19 \mathrm{~h}$. After cooling the reaction to room temperature, 1,3,5-trimethoxybenzene was added to the reaction mixture. The crude mixture was analyzed by ${ }^{1} \mathrm{H}$ NMR spectroscopy showing $7 \%$ of reduced product formation with $93 \%$ of unreacted starting pyridine. 


\subsection{Deuterium Labelling Experiment in the Absence of Hydrogen Gas}
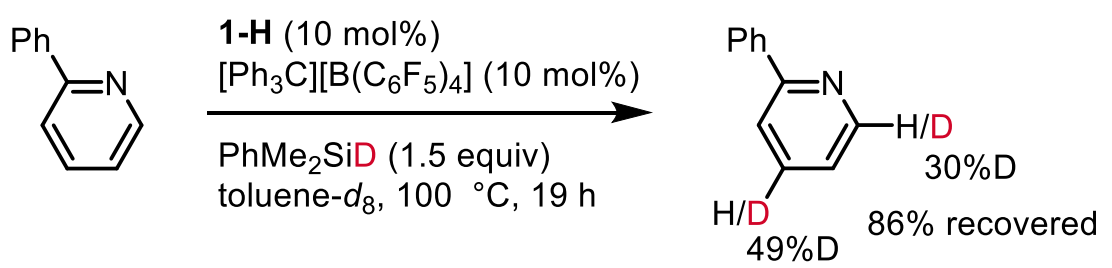

In a glovebox, a 0.5 dram vial was charged with $\left[\mathrm{Ph}_{3} \mathrm{C}\right]\left[\mathrm{B}\left(\mathrm{C}_{6} \mathrm{~F}_{5}\right)_{4}\right](11.5 \mathrm{mg}, 0.0125 \mathrm{mmol})$ and $\mathbf{1}-\mathbf{H}(4.3$ $\mathrm{mg}, 0.0125 \mathrm{mmol}$ ) then $0.5 \mathrm{~mL}$ of toluene- $d_{8}$ was added. The mixture was swirled until the colour changed from red to pale yellow. 2-Phenylpyridine $(19 \mathrm{mg}, 0.125 \mathrm{mmol})$ was added to the solution followed by phenyldimethylsilane- $d(26 \mathrm{mg}, 0.1875 \mathrm{mmol}$ ) added directly to the vial. The solution was heated at 100 ${ }^{\circ} \mathrm{C}$ for $19 \mathrm{~h}$. After cooling the reaction to room temperature, 1,3,5-trimethoxybenzene (13.5 mg) was added to the reaction mixture. Then the crude mixture was analyzed by ${ }^{1} \mathrm{H}$ NMR spectroscopy without water work-up showing no reduced product formation with $93 \%$ of 2-phenyl pyridine. The crude mixture was purified by column chromatography to afford deuterated 2-phenyl pyridine, with deuterium incorporation at four and six positions ( $86 \%$ yield). This result indicates that the boreniuum cation $\mathbf{1}^{+}$catalyzes the hydrosilylation of 2-phenyl pyridine and that this process is reversible.

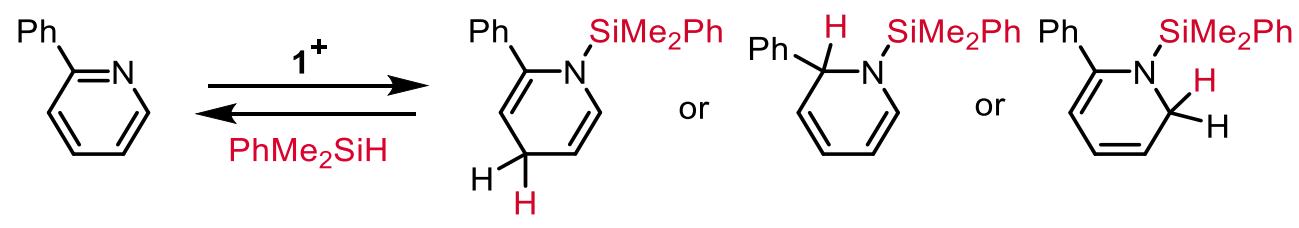



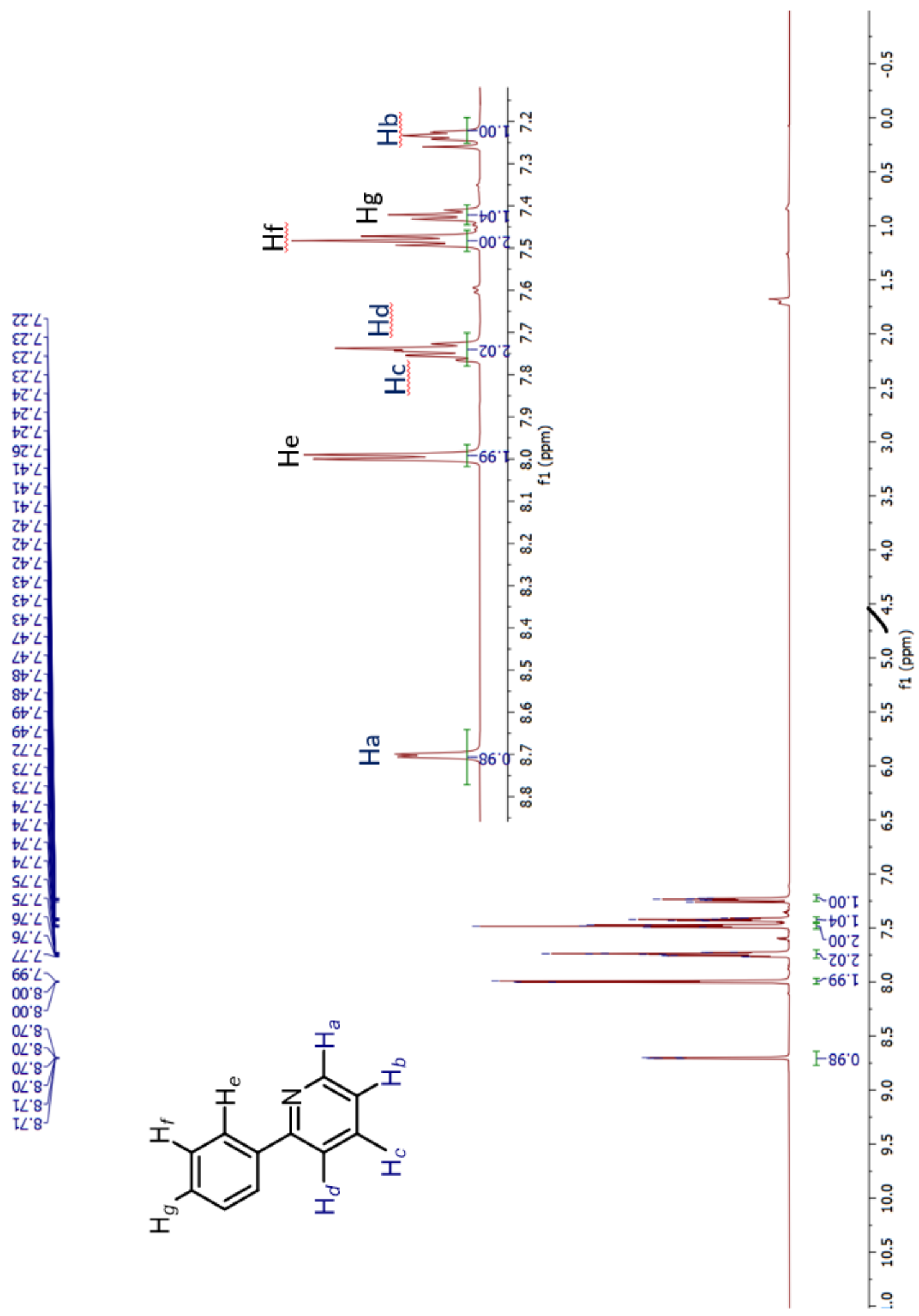

Figure S3. ${ }^{1} \mathrm{H}$ NMR (700 MHz) of 2-phenyl pyridine in $\mathrm{CDCl}_{3}$ 

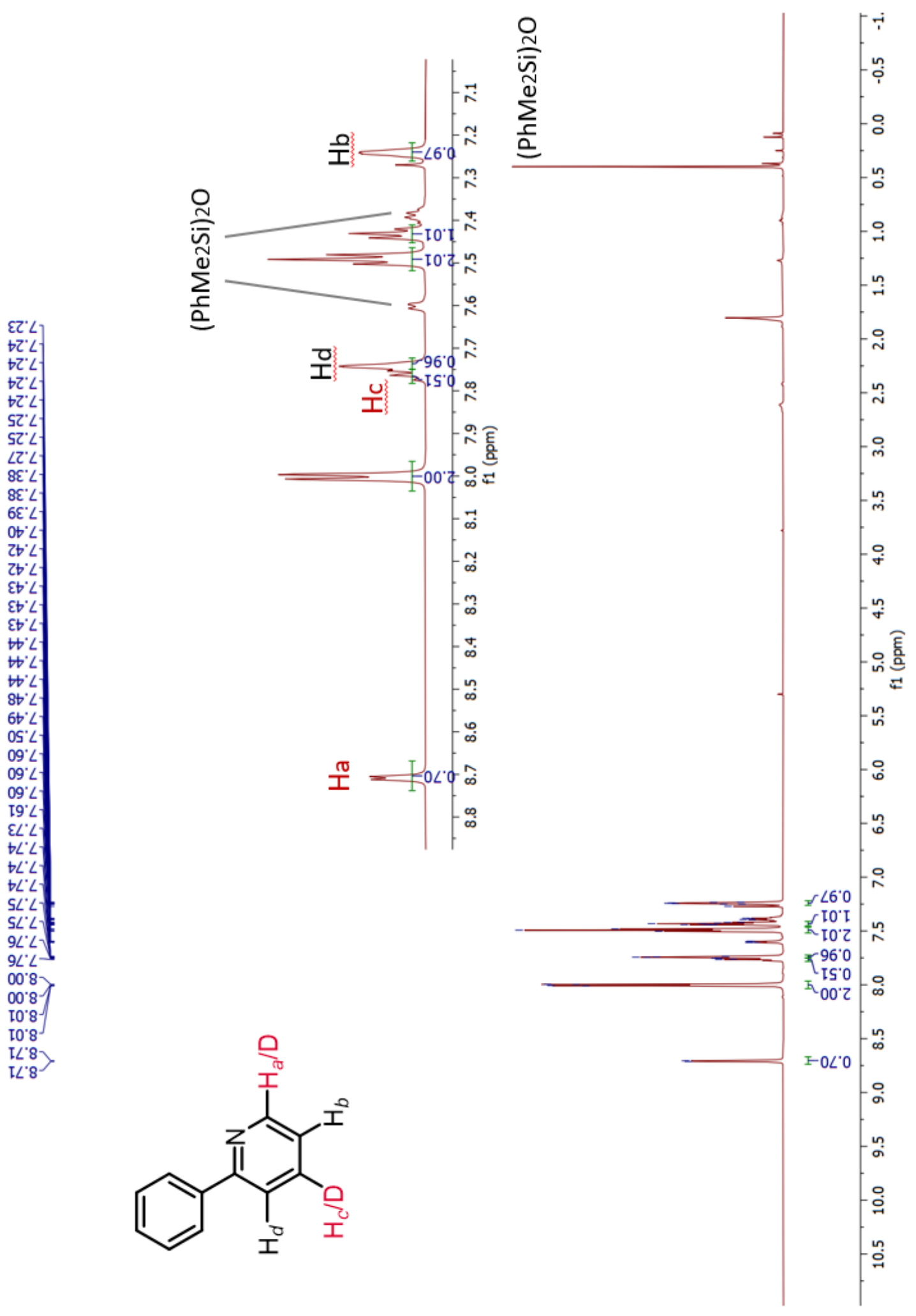

Figure S4. ${ }^{1} \mathrm{H}$ NMR (700 MHz) of deuterated 2-phenyl pyridine in $\mathrm{CDCl}_{3}$ 


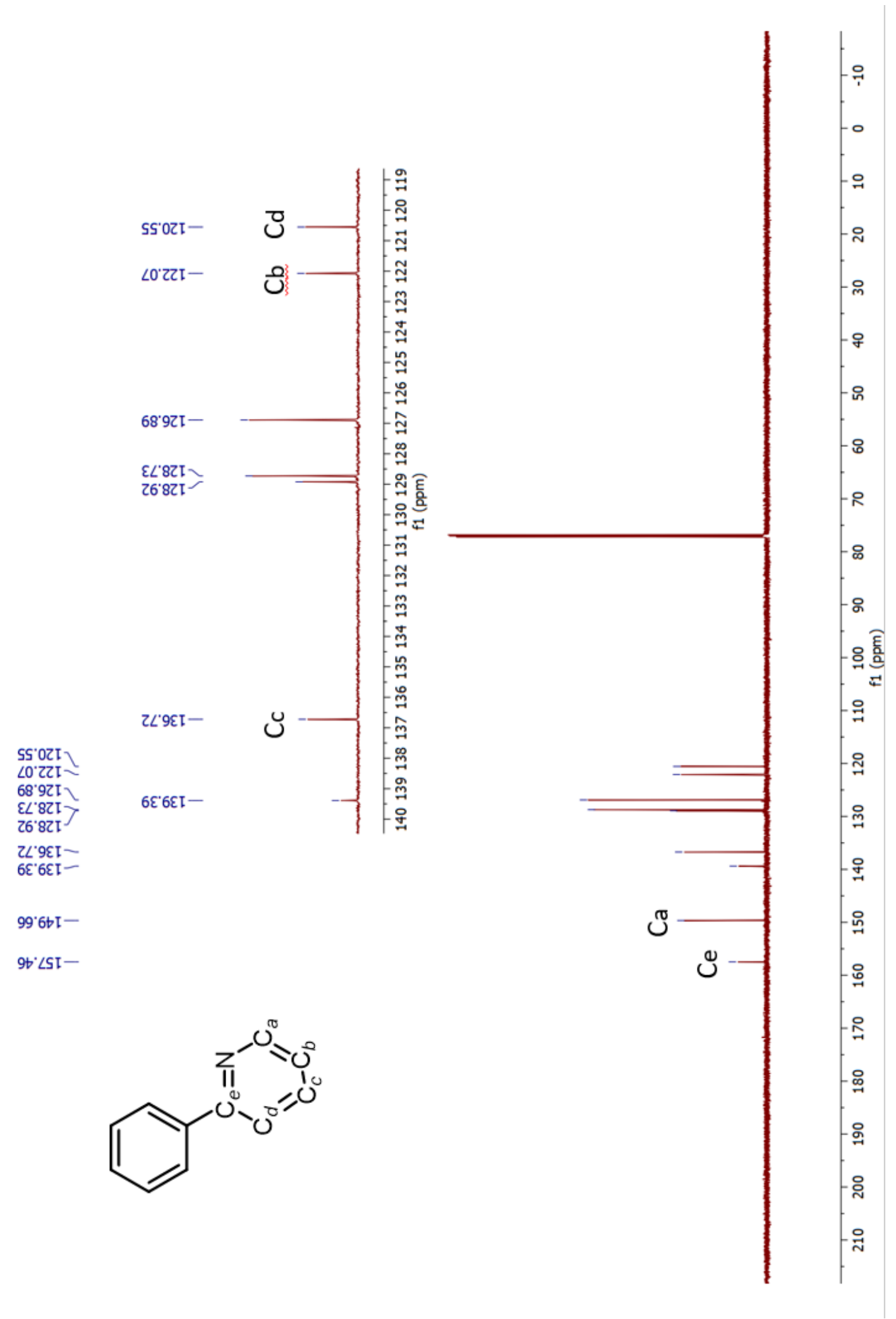

Figure S5. ${ }^{13} \mathrm{C}$ NMR (176 MHz) of 2-phenyl pyridine in $\mathrm{CDCl}_{3}$ 


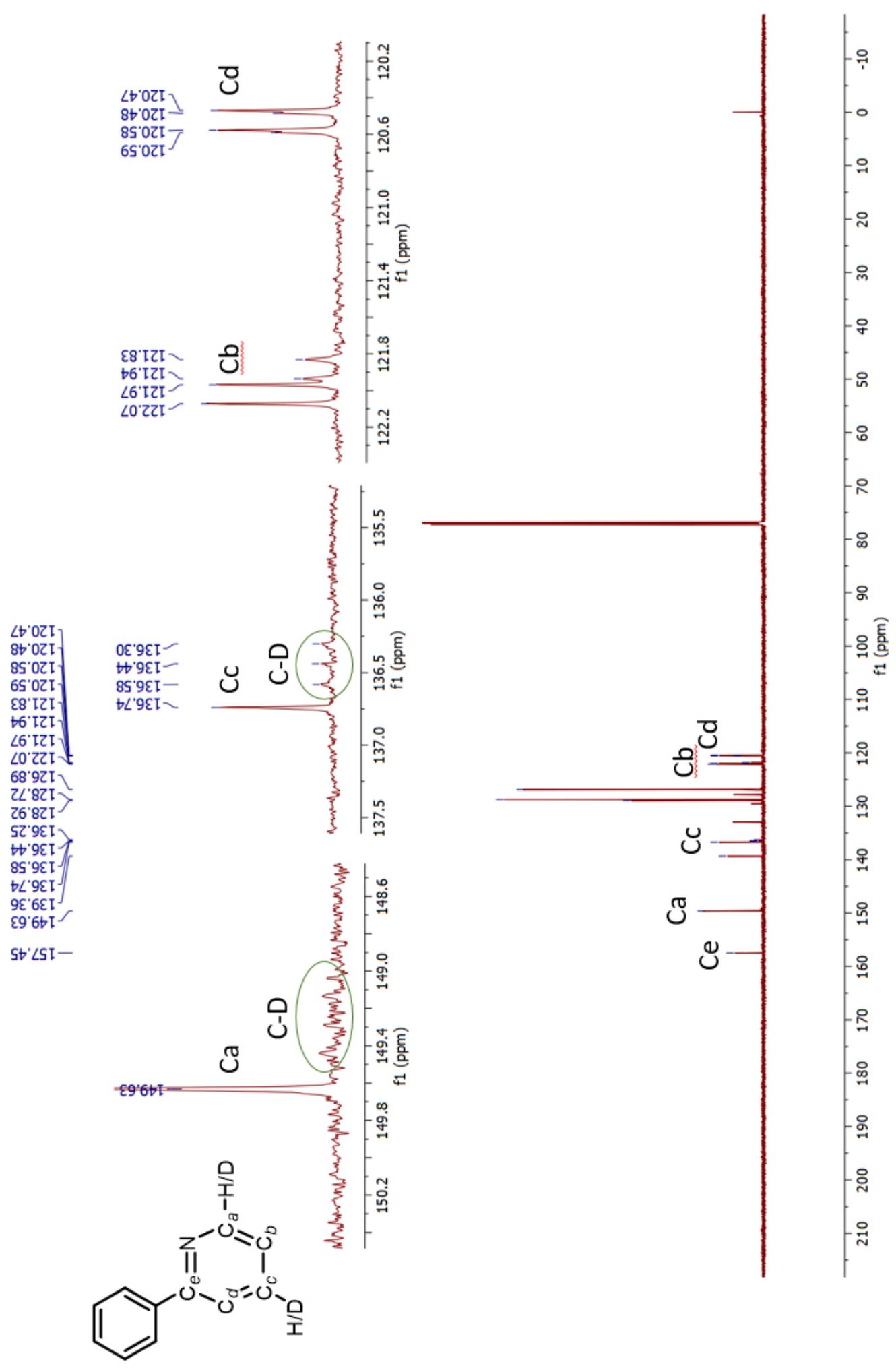

Figure S6. ${ }^{13} \mathrm{C}$ NMR (176 MHz) of deuterated 2-phenyl pyridine in $\mathrm{CDCl}_{3}$ 


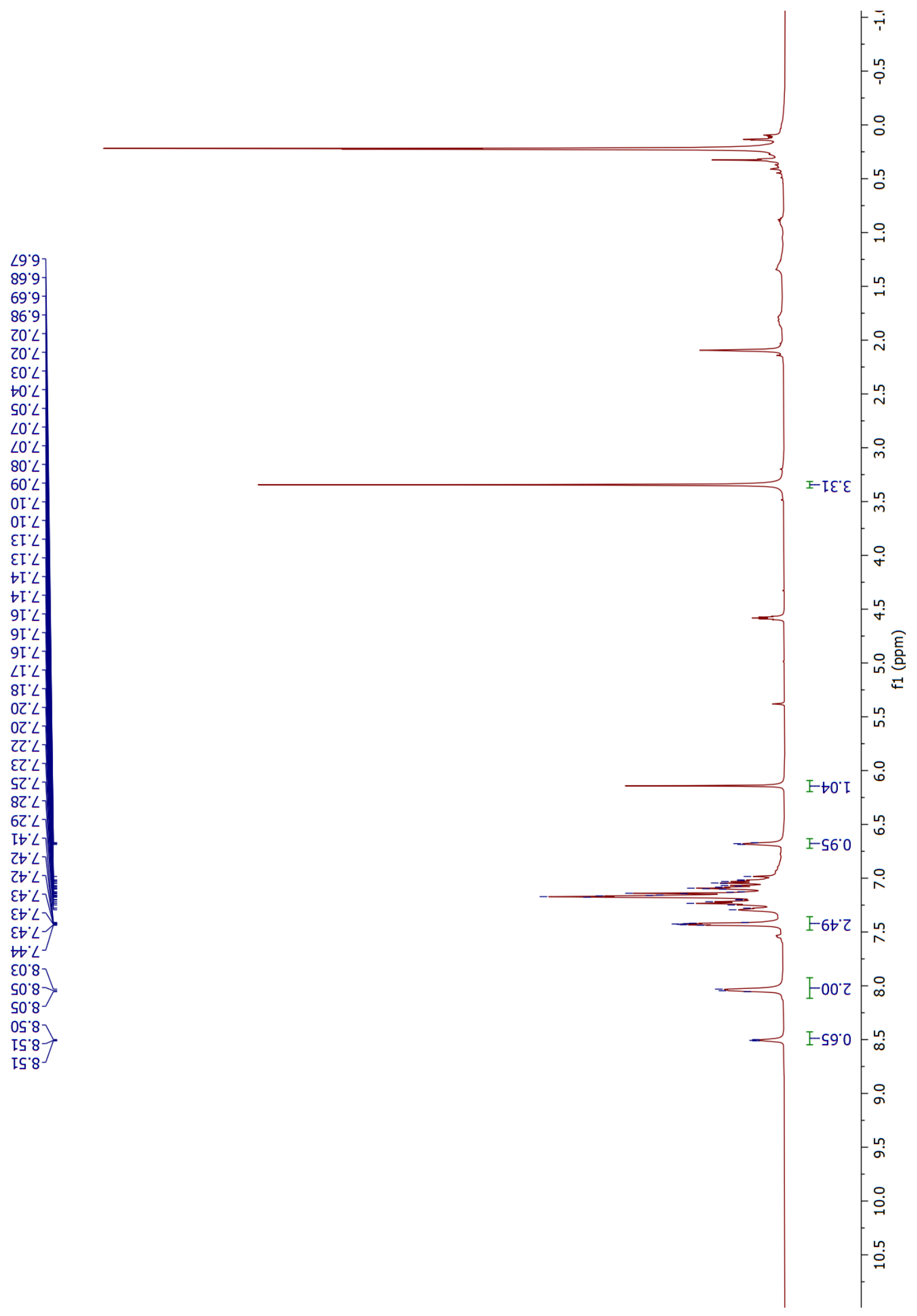

Figure S7. ${ }^{1} \mathrm{H}$ NMR (700 MHz) of a crude reaction mixture in toluene- $d_{8}$ before reaction work-up 


\subsection{Control experiment with excess silane in the absence of hydrogen gas}

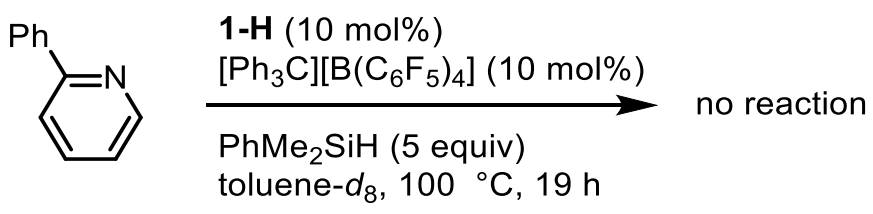

In a glovebox, a 0.5 dram vial was charged with $\left[\mathrm{Ph}_{3} \mathrm{C}\right]\left[\mathrm{B}\left(\mathrm{C}_{6} \mathrm{~F}_{5}\right)_{4}\right](11.5 \mathrm{mg}, 0.02 \mathrm{mmol})$ and $\mathbf{1 - H}(4.3 \mathrm{mg}$, $0.02 \mathrm{mmol}$ ) then $0.5 \mathrm{~mL}$ of toluene- $d_{8}$ was added. The mixture was swirled until the colour changed from red to pale yellow. 2-phenylpyridine $(19 \mathrm{mg}, 0.2 \mathrm{mmol})$ was added to the solution followed by phenyldimethylsilane ( $26 \mathrm{mg}, 1 \mathrm{mmol}$ ) added directly to the vial. The solution was heated at $100{ }^{\circ} \mathrm{C}$ for 19 h. After cooling the reaction to room temperature, 1,3,5-trimethoxybenzene was added to the reaction mixture. Then the crude mixture was analyzed by ${ }^{1} \mathrm{H}$ NMR spectroscopy without water work-up showing no product formation. 


\subsection{Deuterium labelling experiment with $B\left(C_{6} F_{5}\right)_{3}$ as a catalyst in the absence of hydrogen gas}

The reaction with $\mathrm{BCF}\left(\mathrm{B}\left(\mathrm{C}_{6} \mathrm{~F}_{5}\right)_{3}\right)$ was conducted to see if any reduced product can be formed as shown in Chang's $\mathrm{s}^{4}$ report (Scheme 1) or deuterium exchange of 2-phenylpyridine occurs in the presence of BCF.<smiles>Cc1ccccn1</smiles>

1) $\mathrm{B}\left(\mathrm{C}_{6} \mathrm{~F}_{5}\right)_{3}(5 \mathrm{~mol} \%)$ $\mathrm{Et}_{2} \mathrm{SiH}_{2}$ (9 equiv) toluene, $85{ }^{\circ} \mathrm{C}, 18 \mathrm{~h}$

2) $\mathrm{NsCl}$ (1.5 equiv) $\mathrm{NEt}_{3}$ (3 equiv) DMAP (1 $\mathrm{mol} \%)$ $\mathrm{CH}_{2} \mathrm{Cl}_{2}, 0{ }^{\circ} \mathrm{C}$ then r.t., $12 \mathrm{~h}$<smiles></smiles><smiles>CC1CCCCN1N(S)S(=O)(=O)c1ccccc1</smiles><smiles>CS(=O)(=O)c1cccc([N+](=O)[O-])c1</smiles><smiles>Cc1cccnc1-c1ccccc1</smiles>

1) $\mathrm{B}\left(\mathrm{C}_{6} \mathrm{~F}_{5}\right)_{3}(5 \mathrm{~mol} \%)$ $\mathrm{Et}_{2} \mathrm{SiH}_{2}$ (4 equiv) toluene, $110{ }^{\circ} \mathrm{C}, 15 \mathrm{~h}$

2) $\mathrm{PNBCl}$ (1.2 equiv) 1-methylimidazole (1.5 equiv) TMEDA ( 0.2 equiv) $\mathrm{CH}_{2} \mathrm{Cl}_{2}, 0{ }^{\circ} \mathrm{C}$ then r.t., $12 \mathrm{~h}$<smiles>CC[SiH2]C1CC(C)=C(c2ccccc2)N(P(C)(=O)O[Na])C1</smiles><smiles>CC1=C(c2ccccc2)N(P(=O)([O-])O[Na])CCC1</smiles>

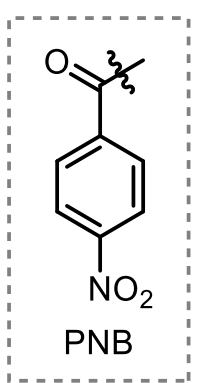

Scheme 1. BCF-Catalyzed reduction of pyridine reported by a Chang group

In a glovebox, a 0.5 dram vial was charged with $\mathrm{B}\left(\mathrm{C}_{6} \mathrm{~F}_{5}\right)_{3}(11.5 \mathrm{mg}, 0.02 \mathrm{mmol})$ and $0.5 \mathrm{~mL}$ of toluene- $d_{8}$ was added. Then, 2-phenylpyridine (19 $\mathrm{mg}, 0.2 \mathrm{mmol})$ was added to the solution followed by phenyldimethylsilane- $d(26 \mathrm{mg}, 0.2 \mathrm{mmol})$ added directly to the vial. The solution was heated at $100{ }^{\circ} \mathrm{C}$ for $19 \mathrm{~h}$. After cooling the reaction to room temperature, 1,3,5-trimethoxybenzene was added to the reaction mixture. Then the crude mixture was analyzed by ${ }^{1} \mathrm{H}$ NMR spectroscopy without water work-up showing $76 \%$ of 2-phenyl pyridine. Then the crude reaction mixture was added to $10 \mathrm{~mL}$ of distilled $\mathrm{H}_{2} \mathrm{O}$ and the aqueous layer was extracted three times with ethyl acetate. The organic fractions were dried over $\mathrm{Na}_{2} \mathrm{SO}_{4}$, filtered, evaporated, and analyzed by GC-MS and ${ }^{1} \mathrm{H}$ NMR. The resulting oil was subjected to preparative thin layer chromatography to give the deuterated starting pyridine (22 mg, 72\%).

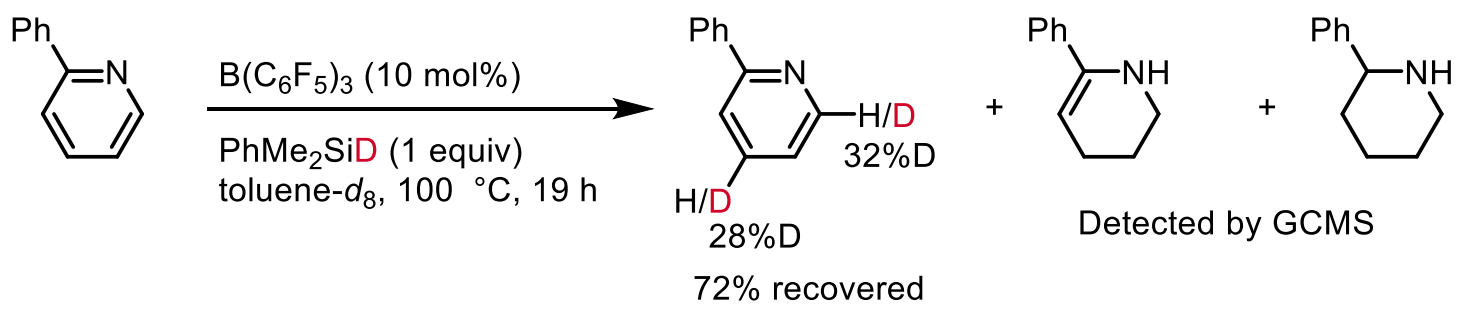




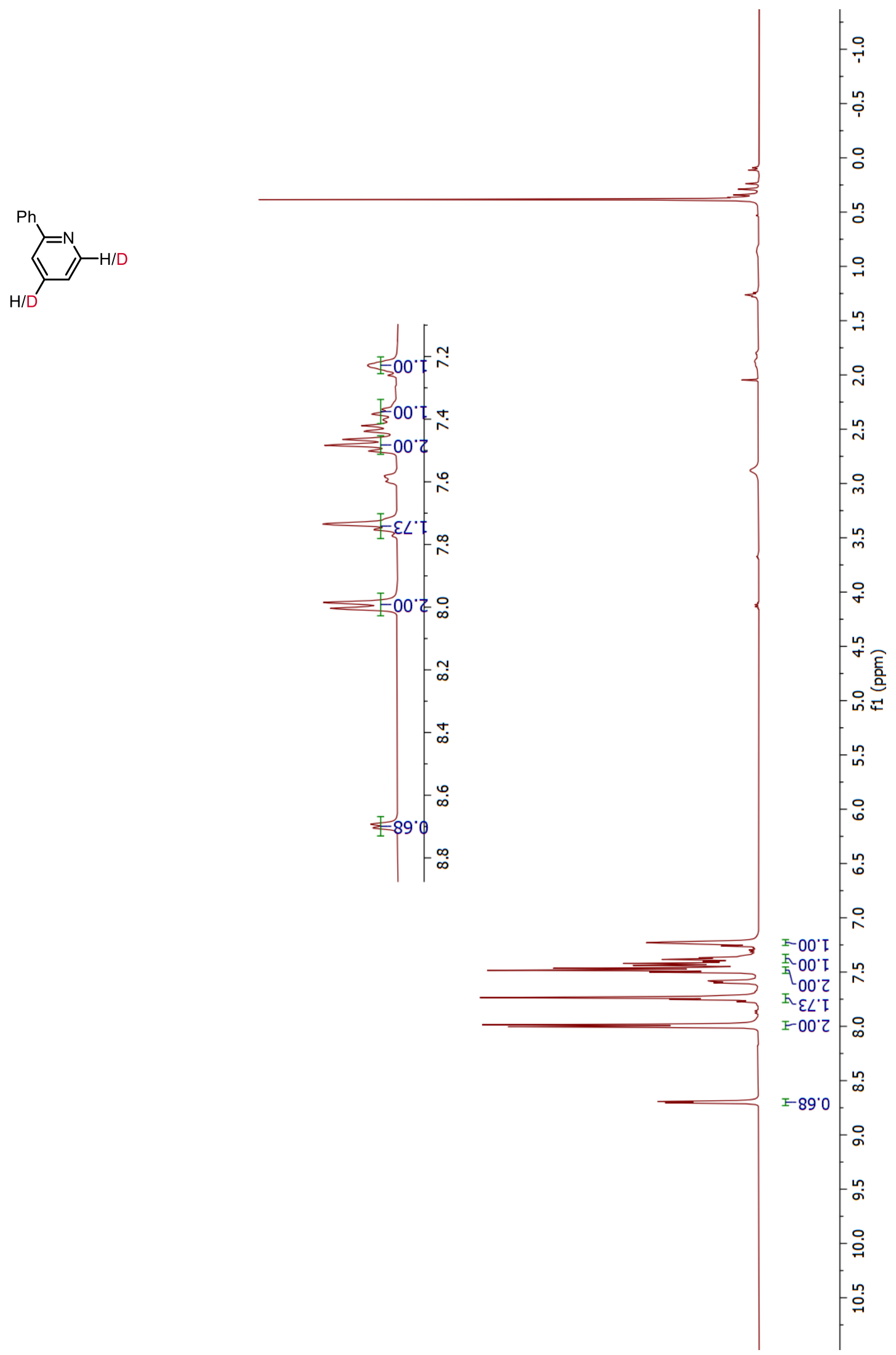

Figure S8. ${ }^{1} \mathrm{H}$ NMR (400 MHz) of purified deuterated 2-phenyl pyridine in $\mathrm{CDCl}_{3}$ 


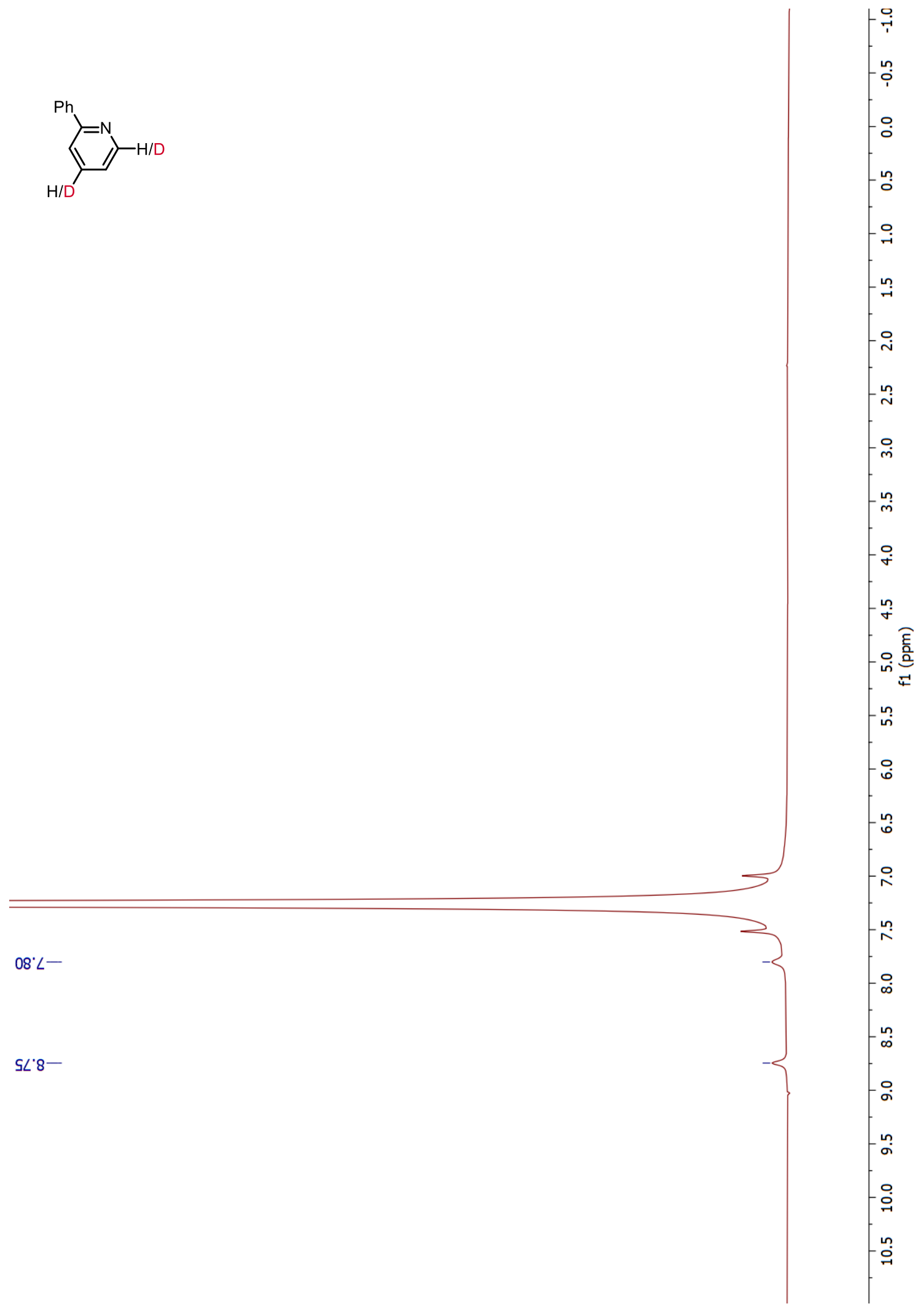

Figure S9. ${ }^{2} \mathrm{H}$ NMR (400 MHz) of purified deuterated 2-phenyl pyridine in $\mathrm{CDCl}_{3}$ 


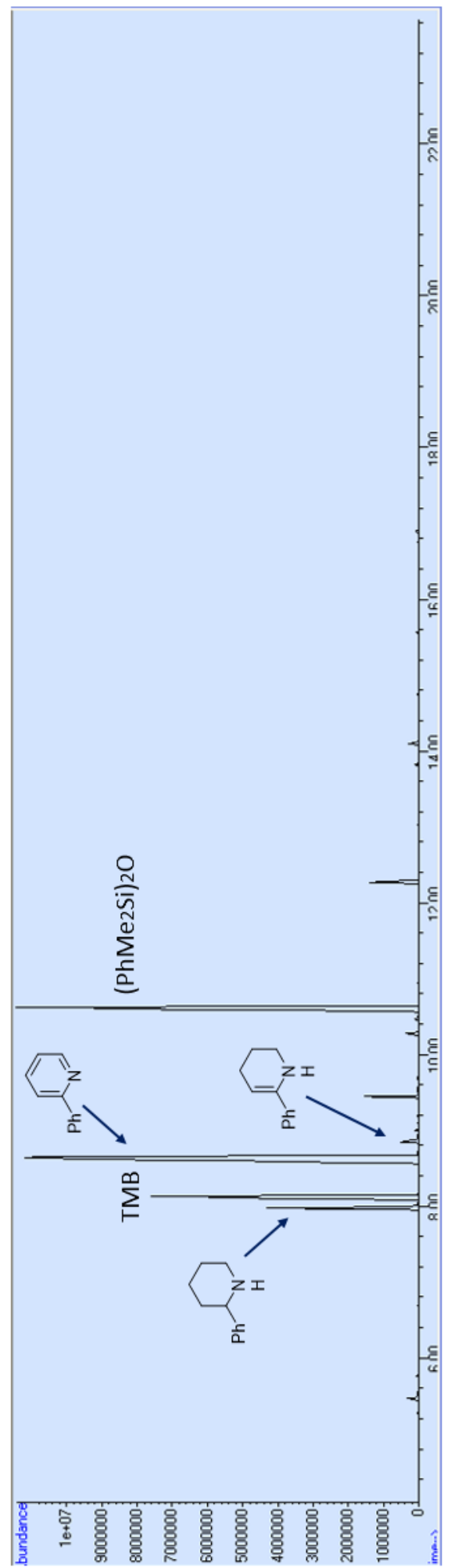

Figure S10. GC-MS analysis of crude mixture after workup with water 


\subsection{Observation of the $N$-silyl protected product without reaction work-up}
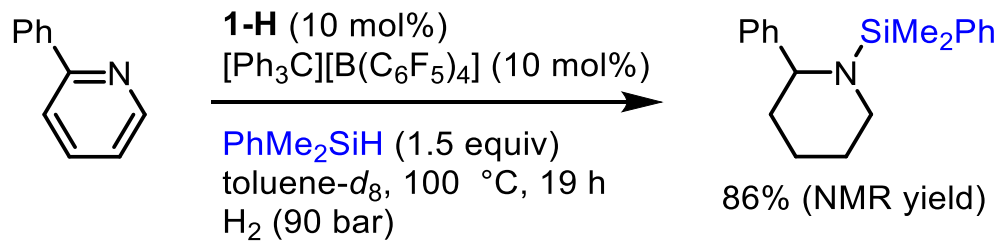

In a glovebox, a 0.5 dram vial was charged with $\left[\mathrm{Ph}_{3} \mathrm{C}\right]\left[\mathrm{B}\left(\mathrm{C}_{6} \mathrm{~F}_{5}\right)_{4}\right](11.5 \mathrm{mg}, 0.0125 \mathrm{mmol})$ and $\mathbf{1 - H}(4.3$ $\mathrm{mg}, 0.0125 \mathrm{mmol}$ ) then $0.5 \mathrm{~mL}$ of toluene- $d_{8}$ was added. The mixture was swirled until the colour changed from red to pale yellow. 2-Phenylpyridine $(19 \mathrm{mg}, 0.125 \mathrm{mmol})$ was added to the solution followed by phenyldimethylesilane $(26 \mathrm{mg}, 0.1875 \mathrm{mmol}$ ) added directly to the vial. The reaction mixture was placed in a $50 \mathrm{~mL}$ stainless steel Parr pressure vessel and sealed with the gas regulator outside the glovebox. The pressure vessel was pressurized and depressurized five times with hydrogen gas (26 bar) then brought up to the required pressure before heating $\left(76\right.$ bar for $100{ }^{\circ} \mathrm{C}$ ). The vessel was then submerged in an oil bath of the proper temperature and heated for $19 \mathrm{~h}$. Afterwards the vessel was slowly depressurized. After cooling the reaction to room temperature, 1,3,5-trimethoxybenzene $(7.7 \mathrm{mg})$ was added to the reaction mixture. The crude mixture was transferred into an NMR tube and analyzed by ${ }^{1} \mathrm{H}$ and ${ }^{13} \mathrm{C}$ NMR spectroscopy without further purification due to the instability of the $N$-silylated product on silica gel and in moisture, which indicated that $N$-silylated product was obtained as a colourless oil in $86 \%$ yield.

${ }^{1}$ H NMR (500 MHz, Toluene- $\left.d_{8}\right): \delta 7.60(\mathrm{~d}, J=6.1 \mathrm{~Hz}, 2 \mathrm{H}), 7.36(\mathrm{~d}, J=7.8 \mathrm{~Hz}, 2 \mathrm{H}), 7.24-7.19$ (m, 4H), 7.11-7.04 (m, 2H), $4.26(\mathrm{t}, J=3.8 \mathrm{~Hz}, 1 \mathrm{H}), 2.82(\mathrm{dd}, J=6.9,3.0 \mathrm{~Hz}, 2 \mathrm{H}), 2.18-1.99(\mathrm{~m}, 1 \mathrm{H}), 1.71-$ $1.59(\mathrm{~m}, 1 \mathrm{H}), 1.46-1.39(\mathrm{~m}, 1 \mathrm{H}), 1.37-1.26(\mathrm{~m}, 1 \mathrm{H}), 1.24-1.18(\mathrm{~m}, 1 \mathrm{H}), 0.35(\mathrm{~s}, 3 \mathrm{H}), 0.31(\mathrm{~s}, 3 \mathrm{H})$.

${ }^{13} \mathbf{C}\left\{{ }^{1} \mathbf{H}\right\}$ NMR (176 MHz, Toluene- $\left.\boldsymbol{d}_{\mathbf{8}}\right): \delta 144.4,139.9,134.1,129.3,128.5,128.0,127.7,126.1,54.2$, $41.3,30.3,27.8,20.8,-1.3,-1.4$. 


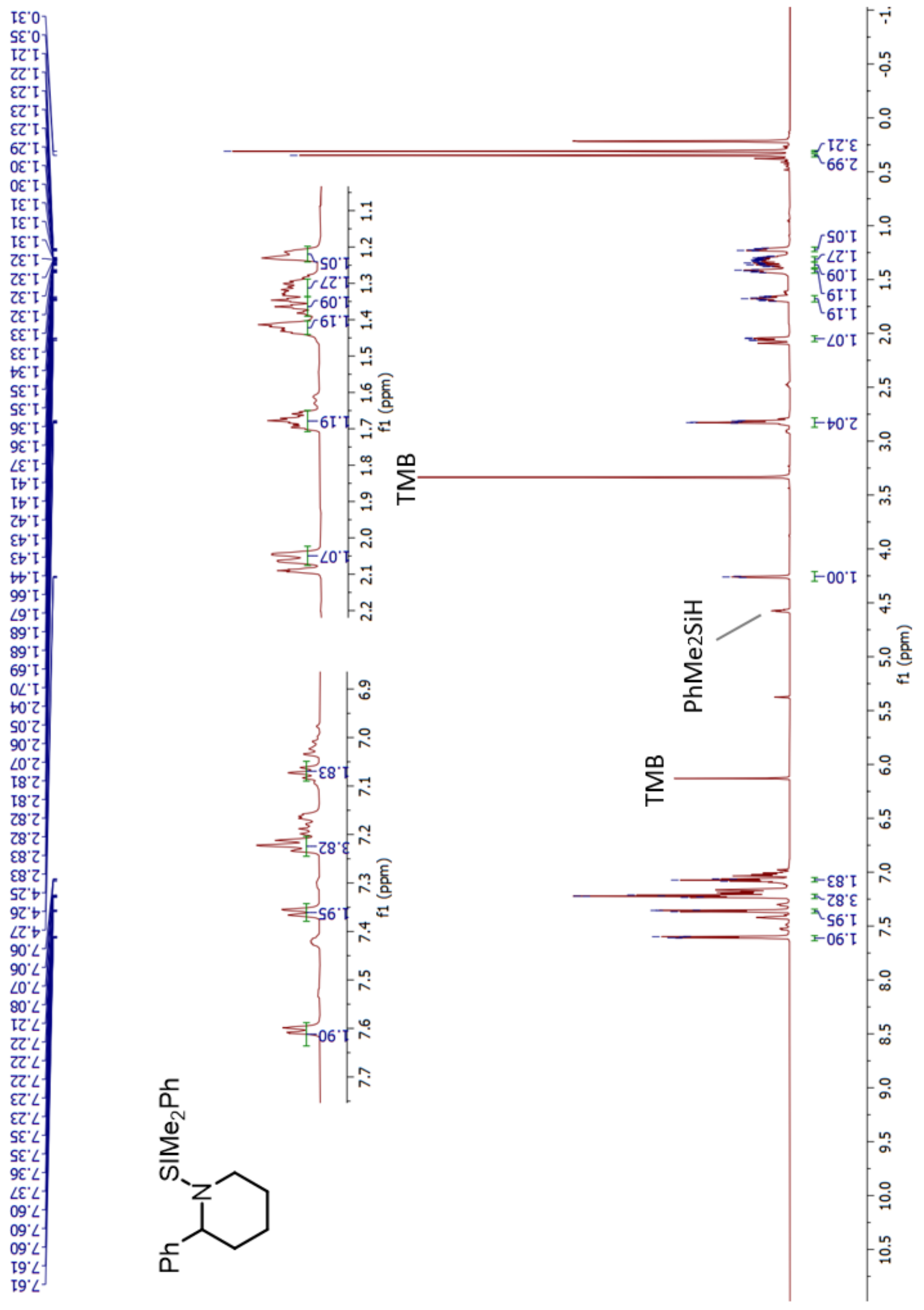

Figure S11. ${ }^{1} \mathrm{H}$ NMR (500 MHz) of $N$-silylated product in toluene- $d_{8}$ 


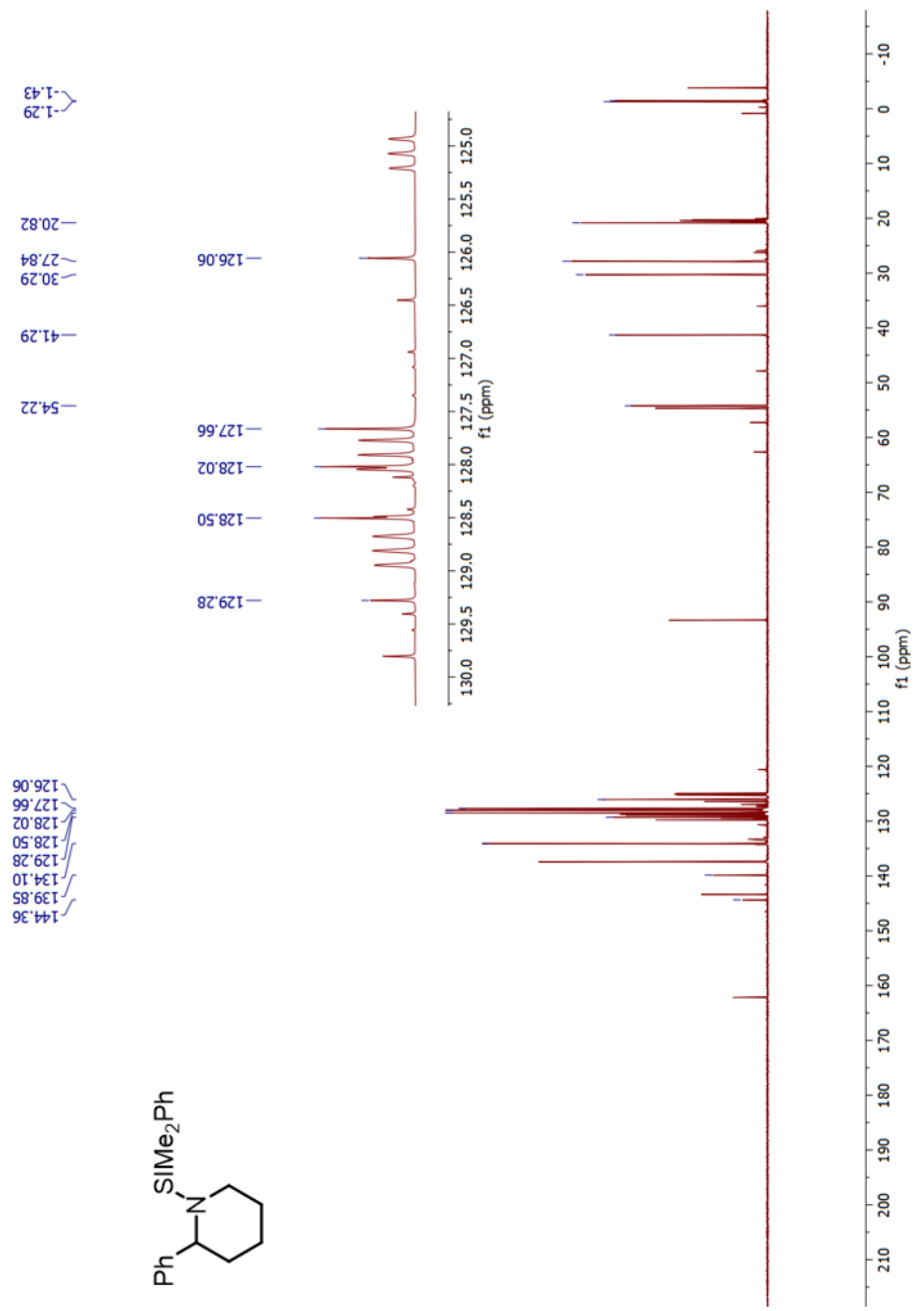

Figure S12. ${ }^{13} \mathrm{C}$ NMR (176 MHz) of $N$-silylated product in toluene- $d_{8}$ 


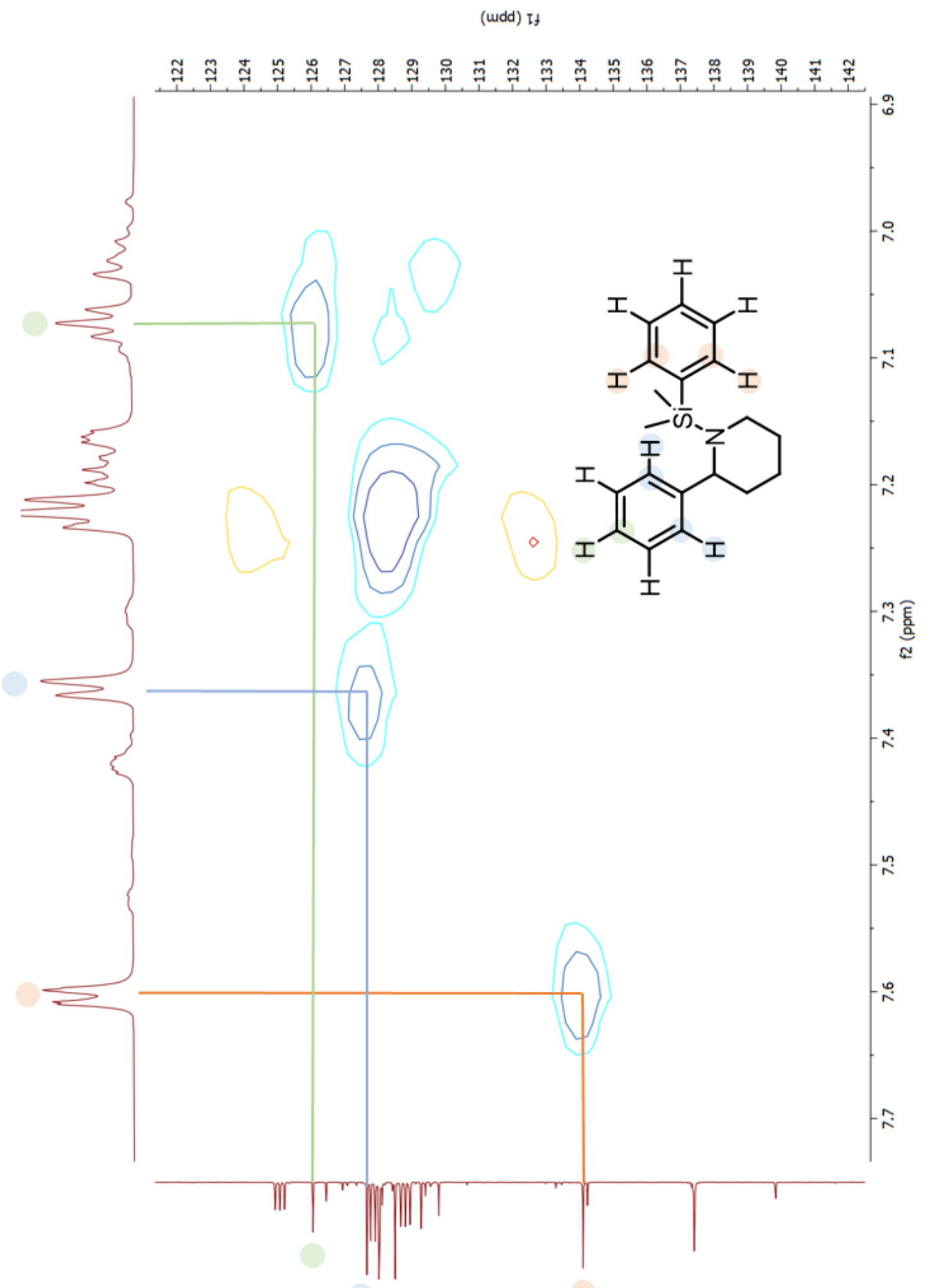

Figure S13. HSQC of $N$-silylated product in toluene- $d_{8}$ 


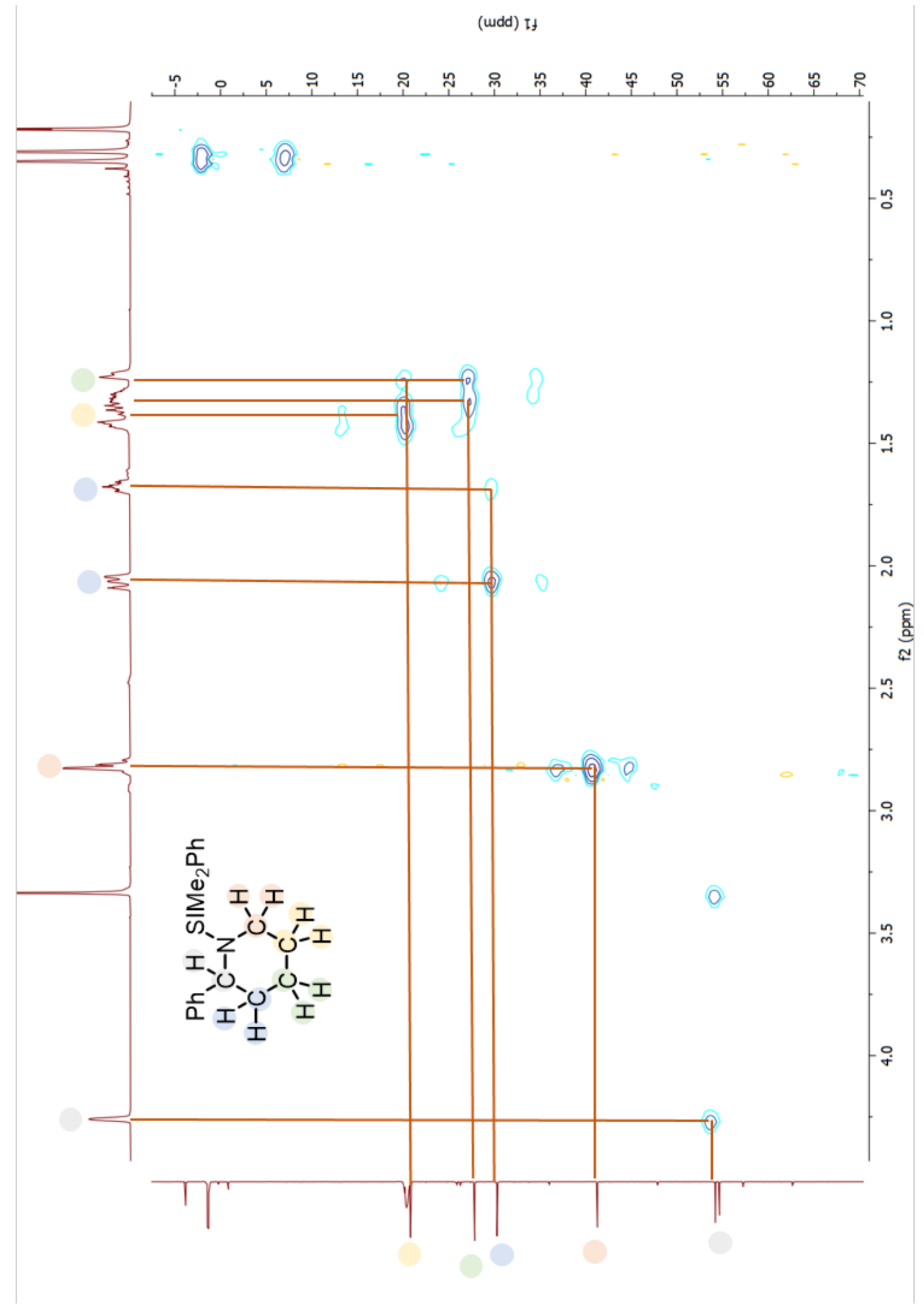

Figure S14. HSQC of $N$-silylated product in toluene- $d_{8}$ 


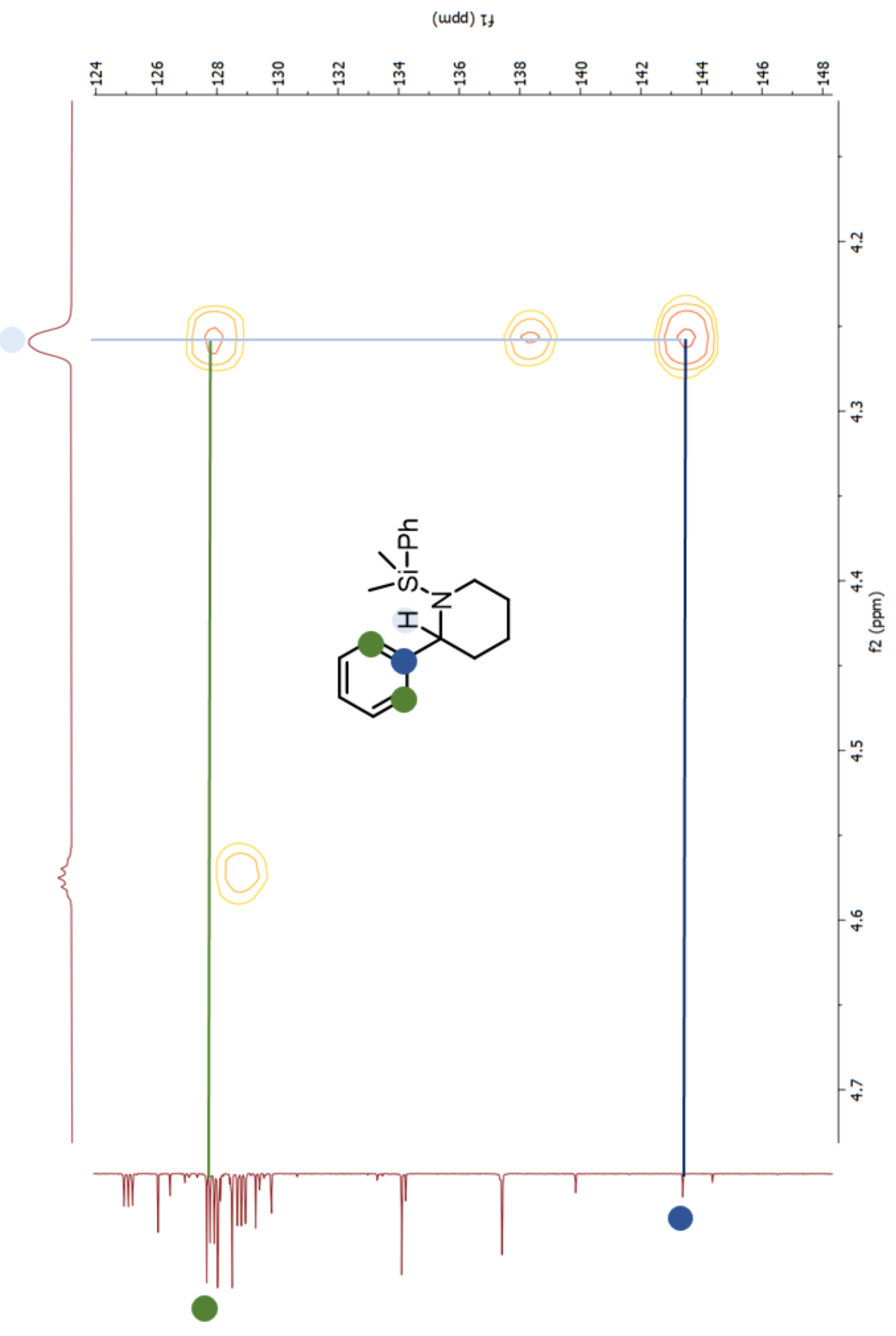

Figure S15. HMBC of $N$-silylated product in toluene- $d_{8}$ 


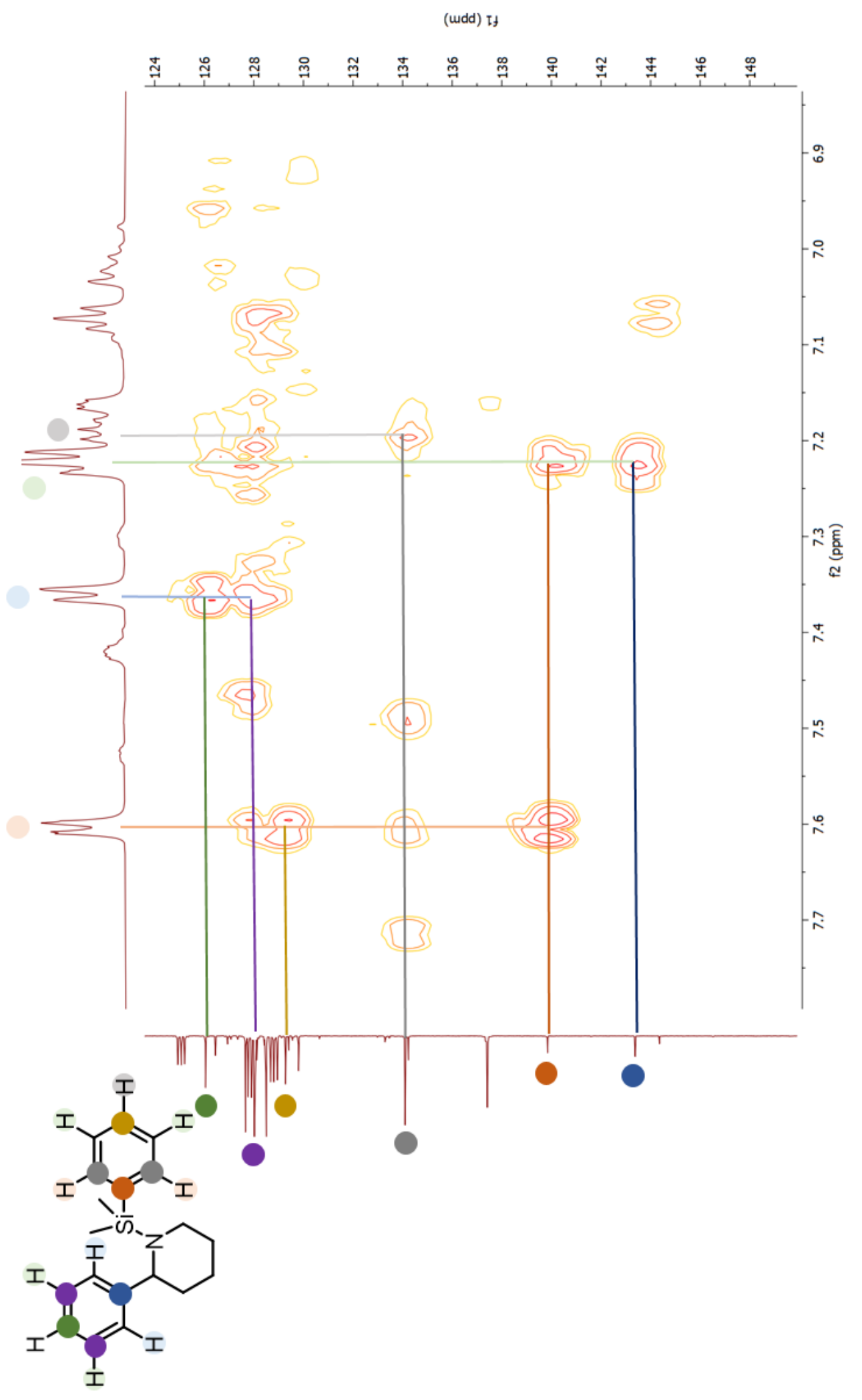

Figure S16. HMBC of $N$-silylated product in toluene- $d_{8}$ 


\subsection{Deuterium labelling experiments}

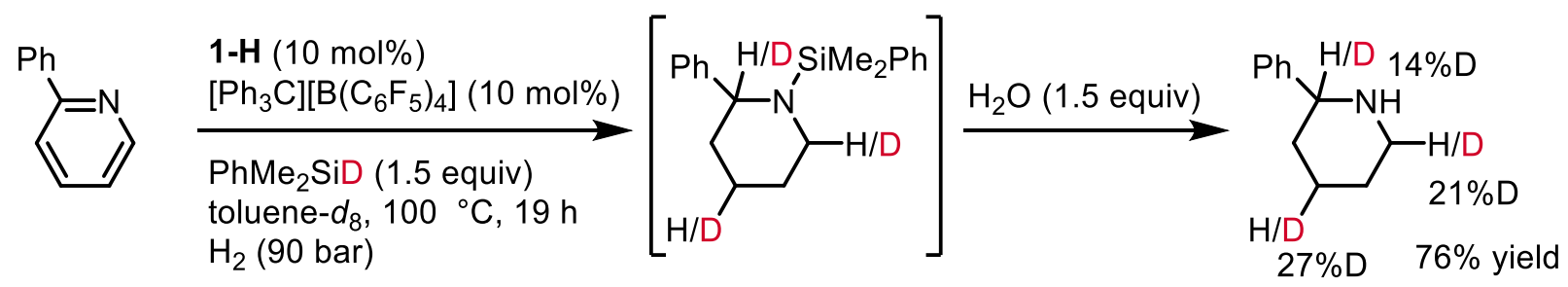

In a glovebox, a 0.5 dram vial was charged with $\left[\mathrm{Ph}_{3} \mathrm{C}\right]\left[\mathrm{B}\left(\mathrm{C}_{6} \mathrm{~F}_{5}\right)_{4}\right](11.5 \mathrm{mg}, 0.0125 \mathrm{mmol})$ and $\mathbf{1 - H}(4.3$ $\mathrm{mg}, 0.0125 \mathrm{mmol}$ ) then $0.5 \mathrm{~mL}$ of toluene- $d_{8}$ was added. The mixture was swirled until the colour changed from red to pale yellow. 2-phenylpyridine $(19 \mathrm{mg}, 0.125 \mathrm{mmol})$ was added to the solution followed by phenyldimethylesilane- $d$ ( $26 \mathrm{mg}, 0.1875 \mathrm{mmol})$ added directly to the vial. The reaction mixture was placed in a $50 \mathrm{~mL}$ stainless steel Parr pressure vessel and sealed with the gas regulator outside the glovebox. The pressure vessel was pressurized and depressurized five times with hydrogen gas (26 bar) then brought up to the required pressure before heating $\left(76\right.$ bar for $\left.100{ }^{\circ} \mathrm{C}\right)$. The vessel was then submerged in an oil bath of the proper temperature and heated for $19 \mathrm{~h}$. Afterwards the vessel was slowly depressurized. After cooling the reaction to room temperature, 1,3,5-trimethoxybenzene was added to the reaction mixture. The crude mixture was transferred into an NMR tube and analyzed by ${ }^{1} \mathrm{H}$ and ${ }^{13} \mathrm{C}$ NMR spectroscopy, which indicated that $N$-silylated product was obtained. The crude mixture was treated with water $(3 \mu \mathrm{L}, 0.18$ $\mathrm{mmol}$ ) and purified by column chromatography on silica gel to give the deuterated piperidine in $76 \%$ yield, which is then analyzed by ${ }^{1} \mathrm{H}$ and ${ }^{2} \mathrm{H}$ NMR spectroscopy. To remove the overlapped N-H signal, the product was further analyzed by ${ }^{1} \mathrm{H}$ and ${ }^{13} \mathrm{C}$ NMR analysis in $\mathrm{CDCl}_{3} / \mathrm{D}_{2} \mathrm{O}(0.5 \mathrm{~mL}: 0.05 \mathrm{~mL})$. 


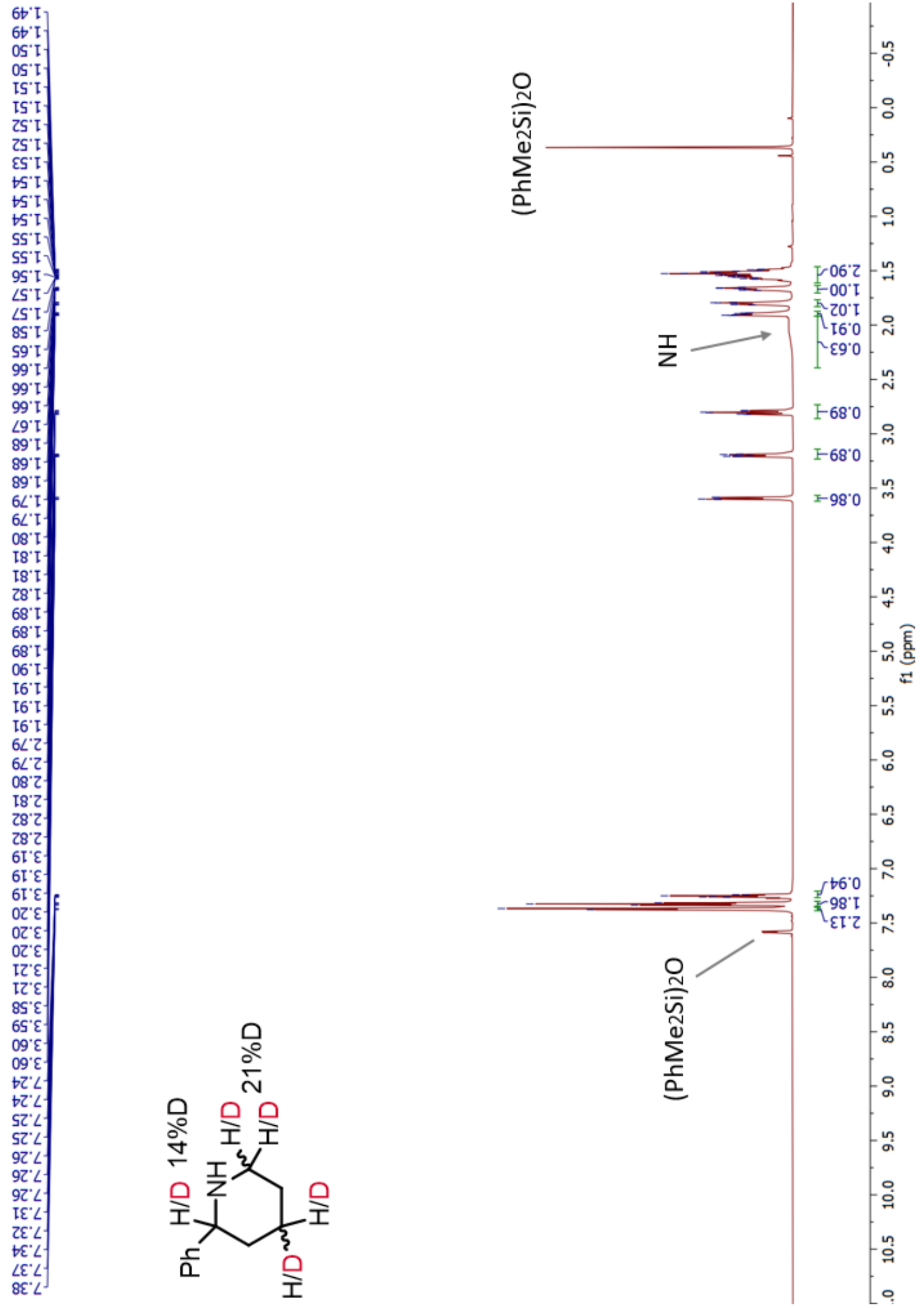

Figure S17. ${ }^{1} \mathrm{H}$ NMR (700 MHz) of deuterated product in $\mathrm{CDCl}_{3}$ 


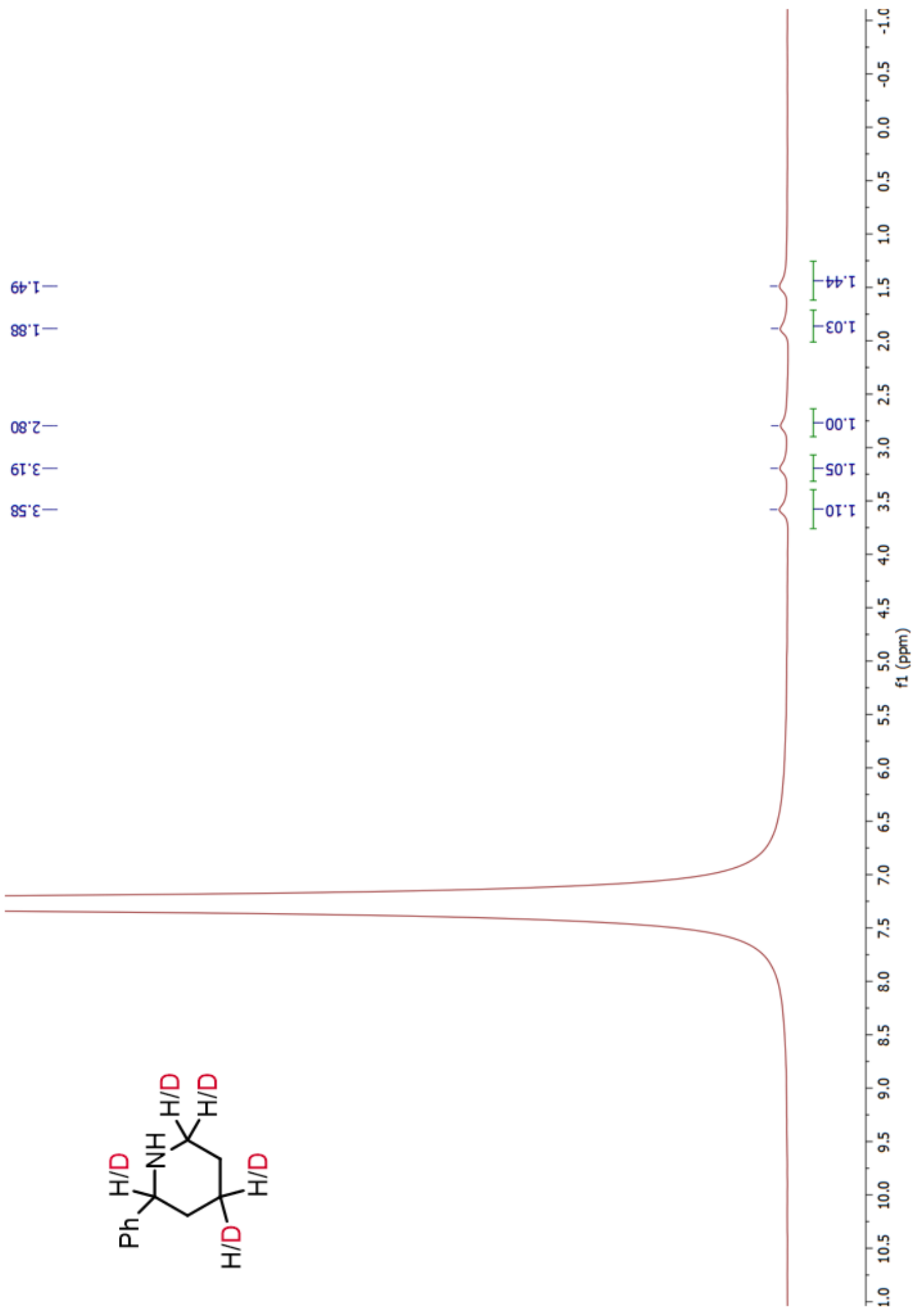

Figure S18. ${ }^{2} \mathrm{H}$ NMR (400 MHz) of deuterated product in $\mathrm{CDCl}_{3}$ 

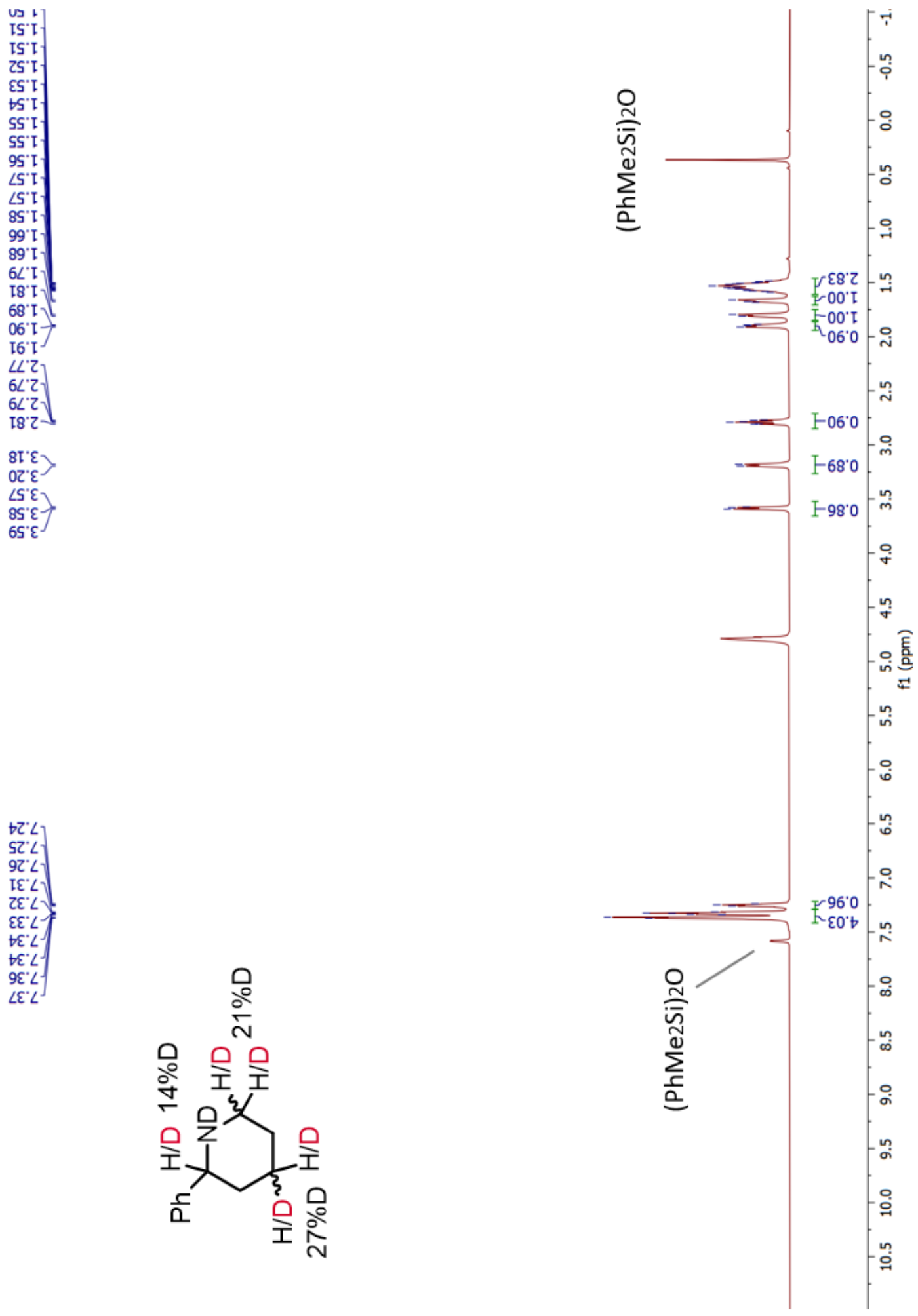

Figure S19. ${ }^{1} \mathrm{H}$ NMR (700 MHz) of deuterated product in $\mathrm{CDCl}_{3} / \mathrm{D}_{2} \mathrm{O}$ 


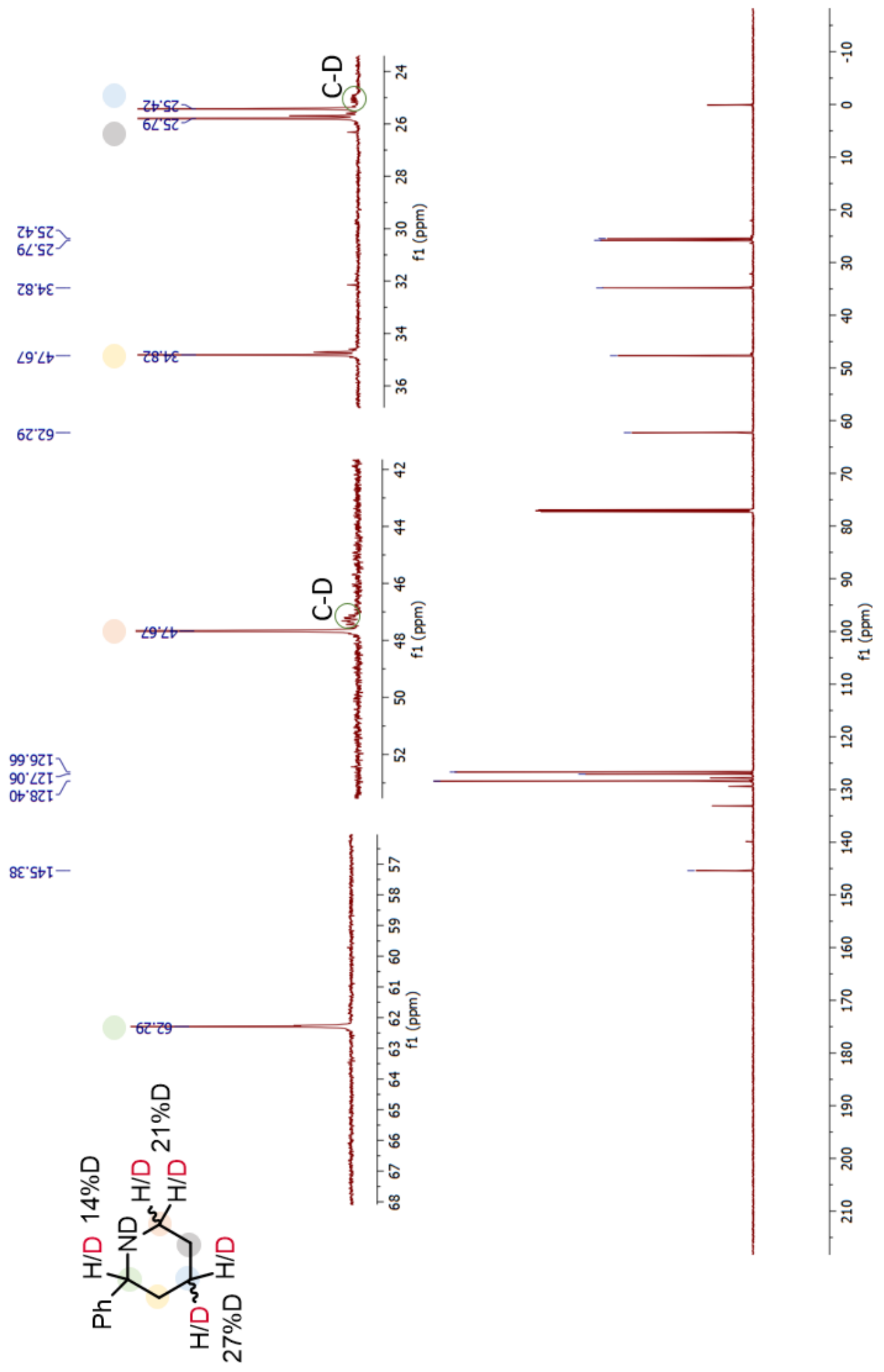

Figure S20. ${ }^{13} \mathrm{C}$ NMR (176 MHz) of deuterated product in $\mathrm{CDCl}_{3} / \mathrm{D}_{2} \mathrm{O}$ 
(udd) If

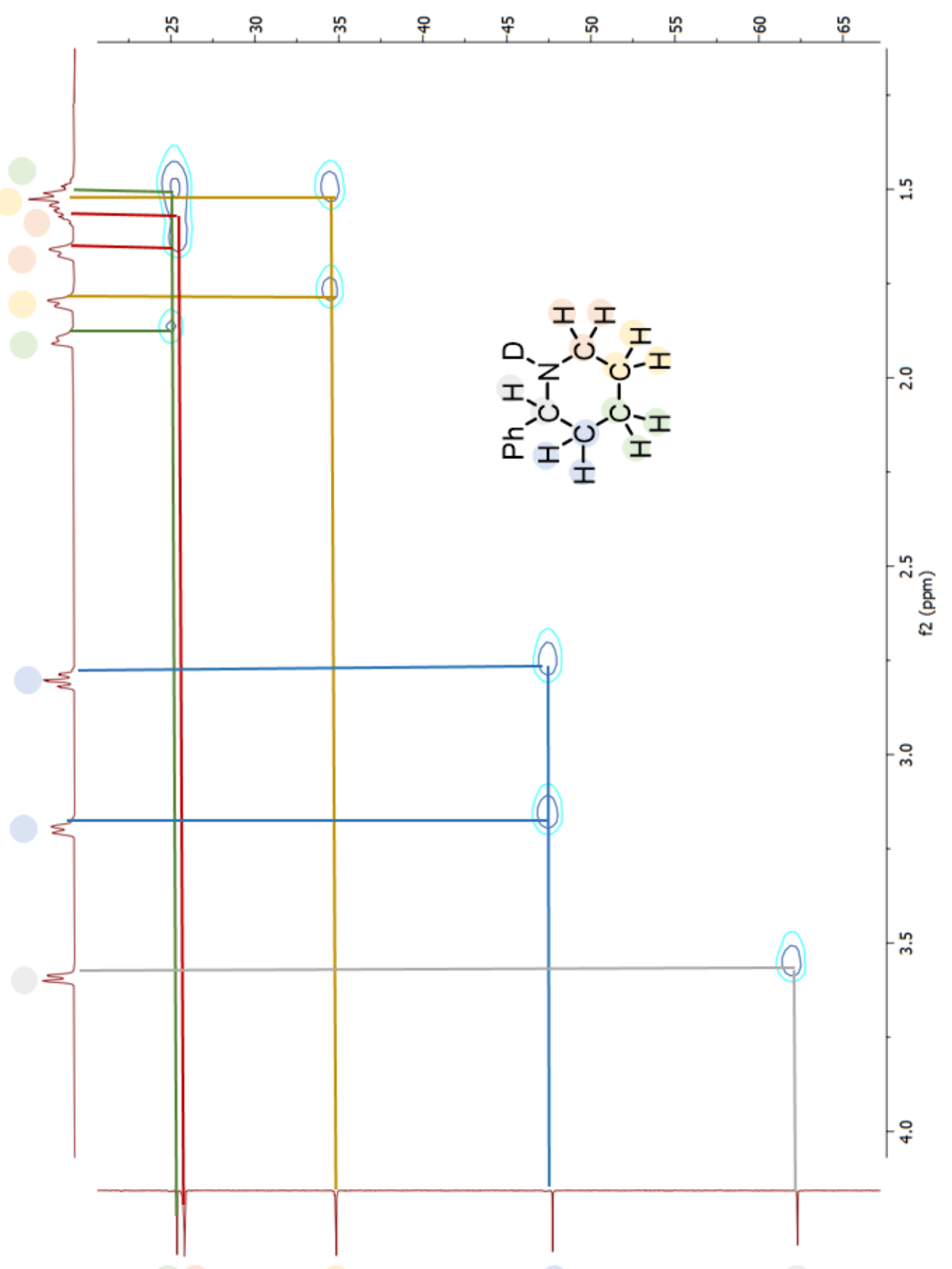

Figure S21. HMBC of deuterated product in $\mathrm{CDCl}_{3} / \mathrm{D}_{2} \mathrm{O}$ 


\section{References}

[1] Labonne, A.; Kribber, T.; Hintermann, L., Highly Active in Situ Catalysts for AntiMarkovnikov Hydration of Terminal Alkynes. Org. Lett. 2006, 8, 5853-5856.

[2] Bielawski, M., Aili, D., and Olofsson, B., Regiospecific One-Pot Synthesis of Diaryliodonium Tetrafluoroborates from Arylboronic Acids and Aryl Iodides, J. Org. Chem. 2008, 73, 12, 4602-4607.

[3] Eisenberger, P., Bestvater, B. P., Keske, E. C., and Crudden, C. M., Hydrogenations at room temperature and atmospheric pressure with mesoionic carbene-stabilized borenium catalysts, Angew. Chem. Int. Ed. 2015, 54, 2467-2471.

[4] N. Gandhamsetty, S. Park and S. Chang, Selective Silylative Reduction of Pyridines Leading to Structurally Diverse Azacyclic Compounds with the Formation of $\mathrm{sp}^{3} \mathrm{C}-\mathrm{Si}$ Bonds, J. Am. Chem. Soc. 2015, 137, 15176-15184. 
8. ${ }^{1} \mathrm{H}$ and ${ }^{13} \mathrm{C}$ NMR Spectra of Products<smiles>Cc1cccc(-c2ccccc2)n1</smiles>

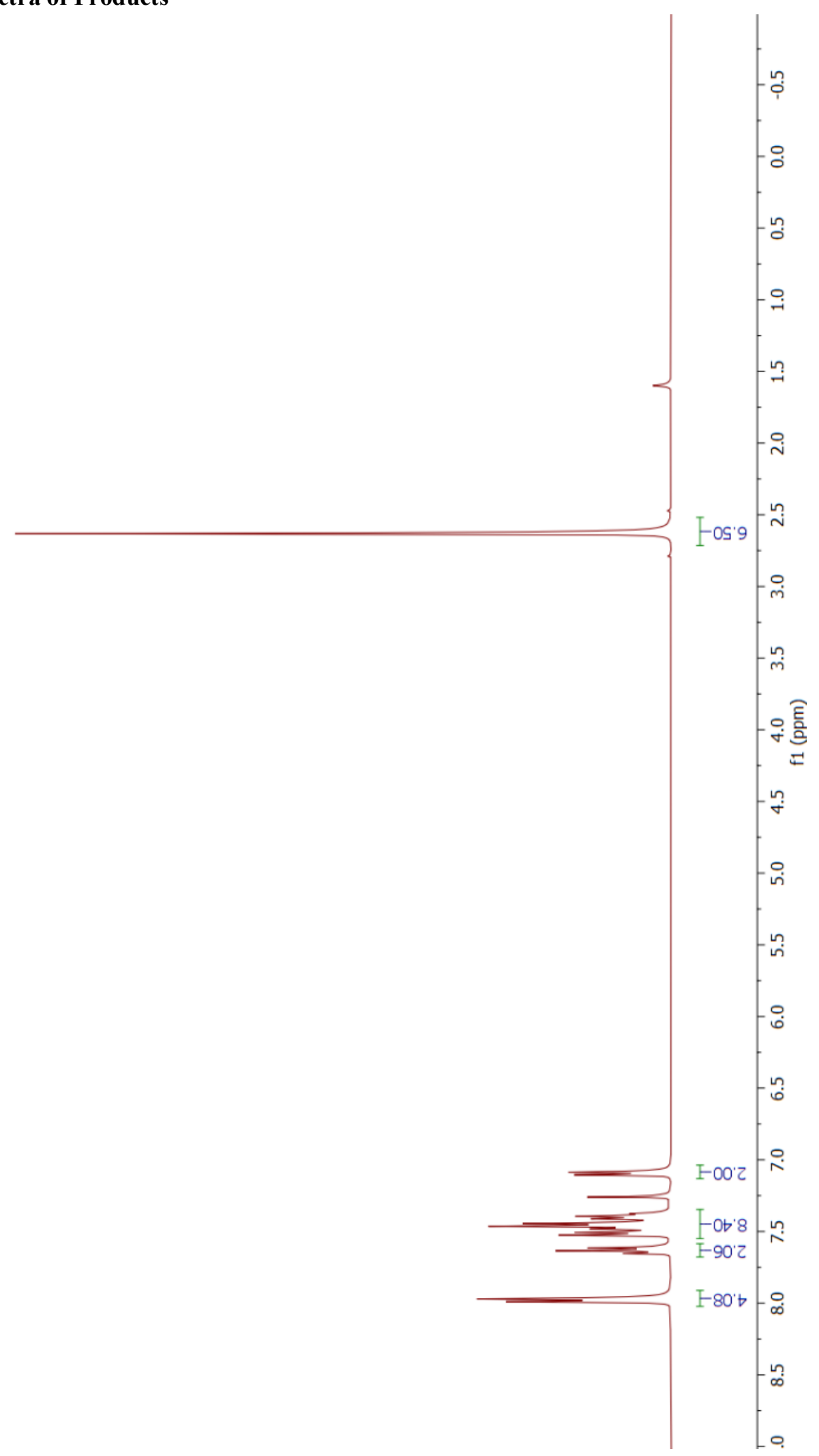

Figure S22. ${ }^{1} \mathrm{H}$ NMR (400 MHz) in $\mathrm{CDCl}_{3} 7 \mathrm{a}$ 


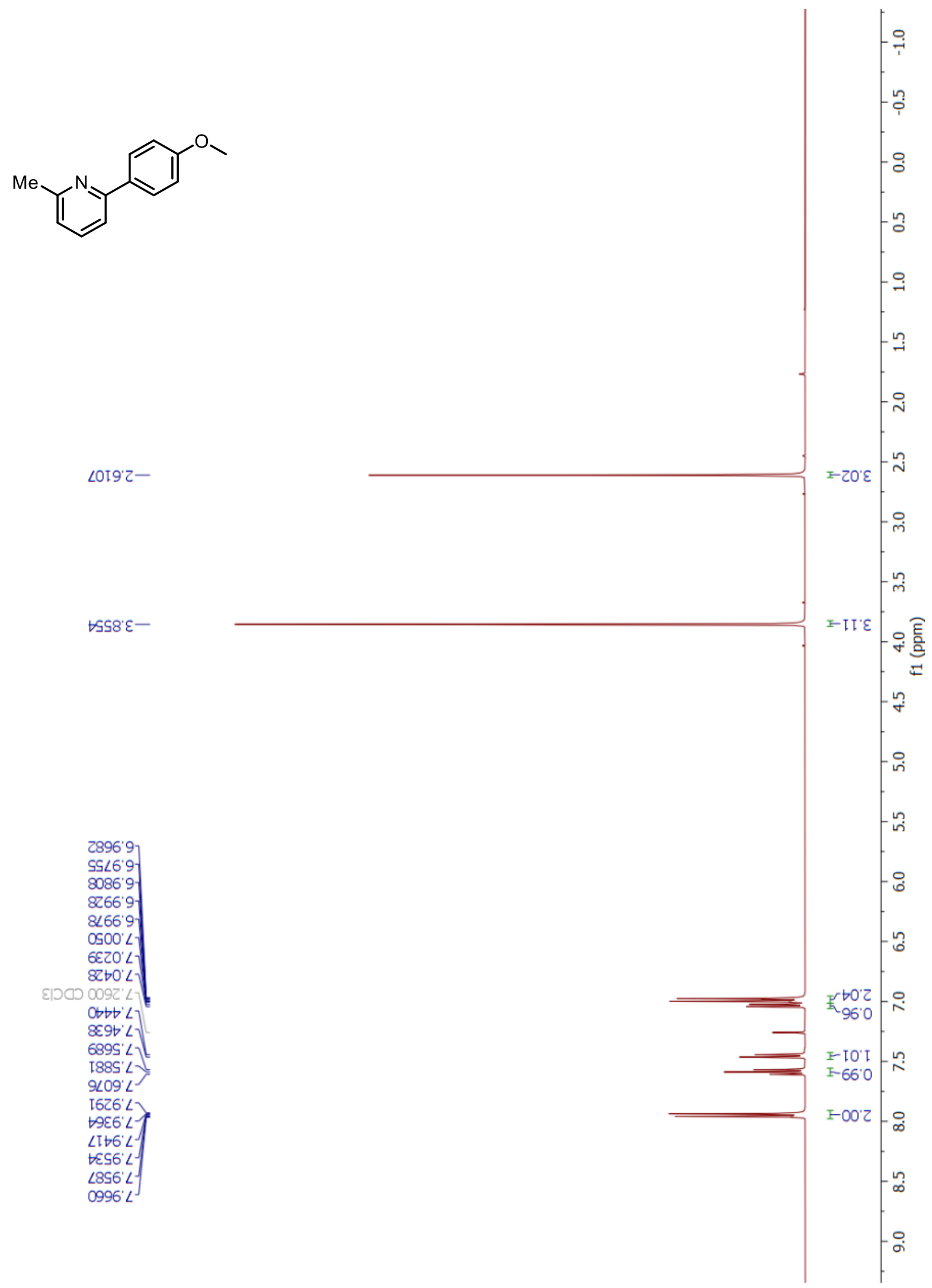

Figure S23. ${ }^{1} \mathrm{H}$ NMR (400 MHz) in $\mathrm{CDCl}_{3} 7 \mathrm{~b}$ 


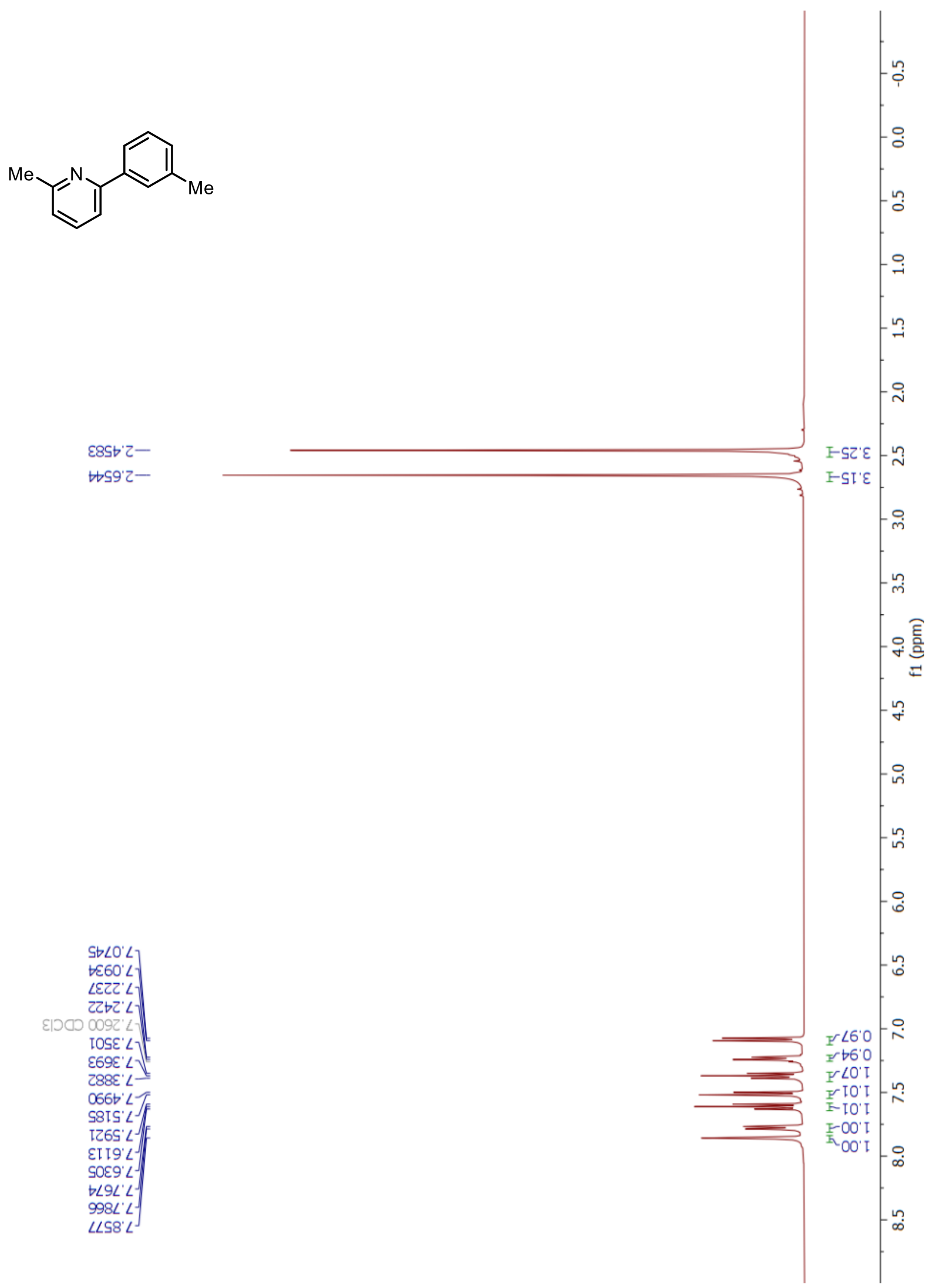

Figure S24. ${ }^{1} \mathrm{H}$ NMR (400 MHz) in $\mathrm{CDCl}_{3} 7 \mathrm{c}$ 
<smiles>Cc1cccc(-c2cccc(C)n2)c1</smiles>

Ot09' $12-$

$8 z 18 \cdot b z-$

I9IL' $\angle I T 工$

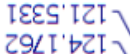

$818 L ' \angle Z I$

OEI9'8ZI -

StZS' 62I

LLE8'9EI)

6908' $8 \mathrm{LI}-$

StE8' $6 E I^{-}$

SEIZ' $\angle S I)$

S†IE' 8SI -

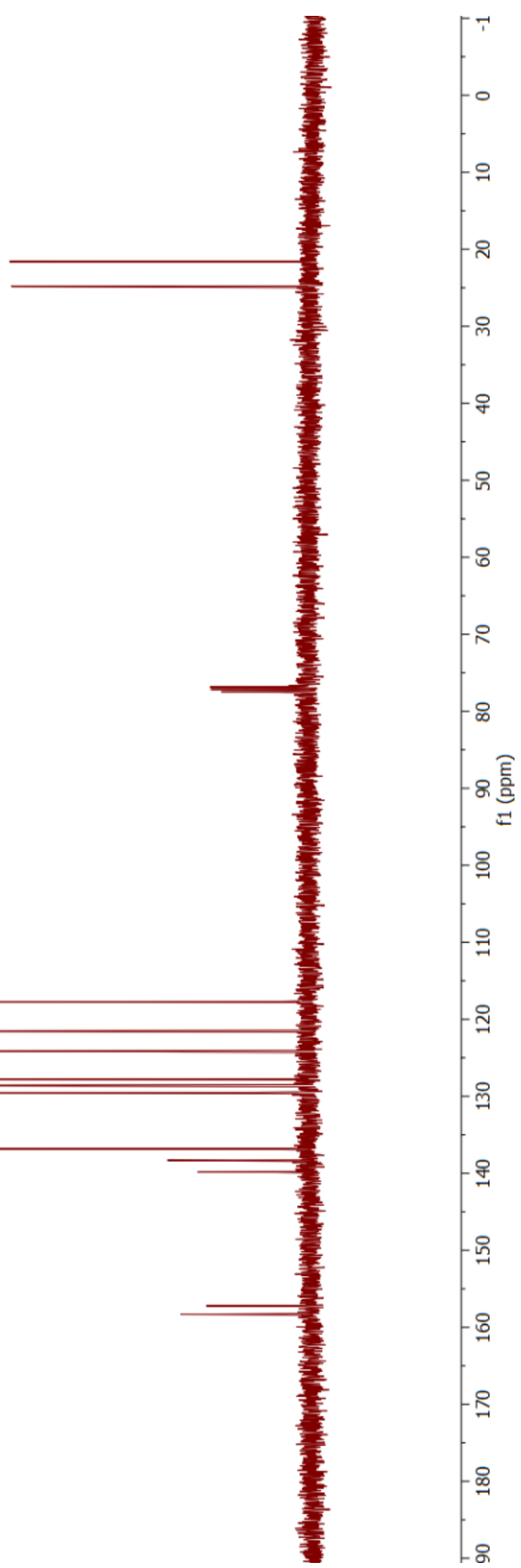

Figure S25. ${ }^{13} \mathrm{C}\left\{{ }^{1} \mathrm{H}\right\}$ NMR (101 MHz) in $\mathrm{CDCl}_{3} 7 \mathrm{c}$ 


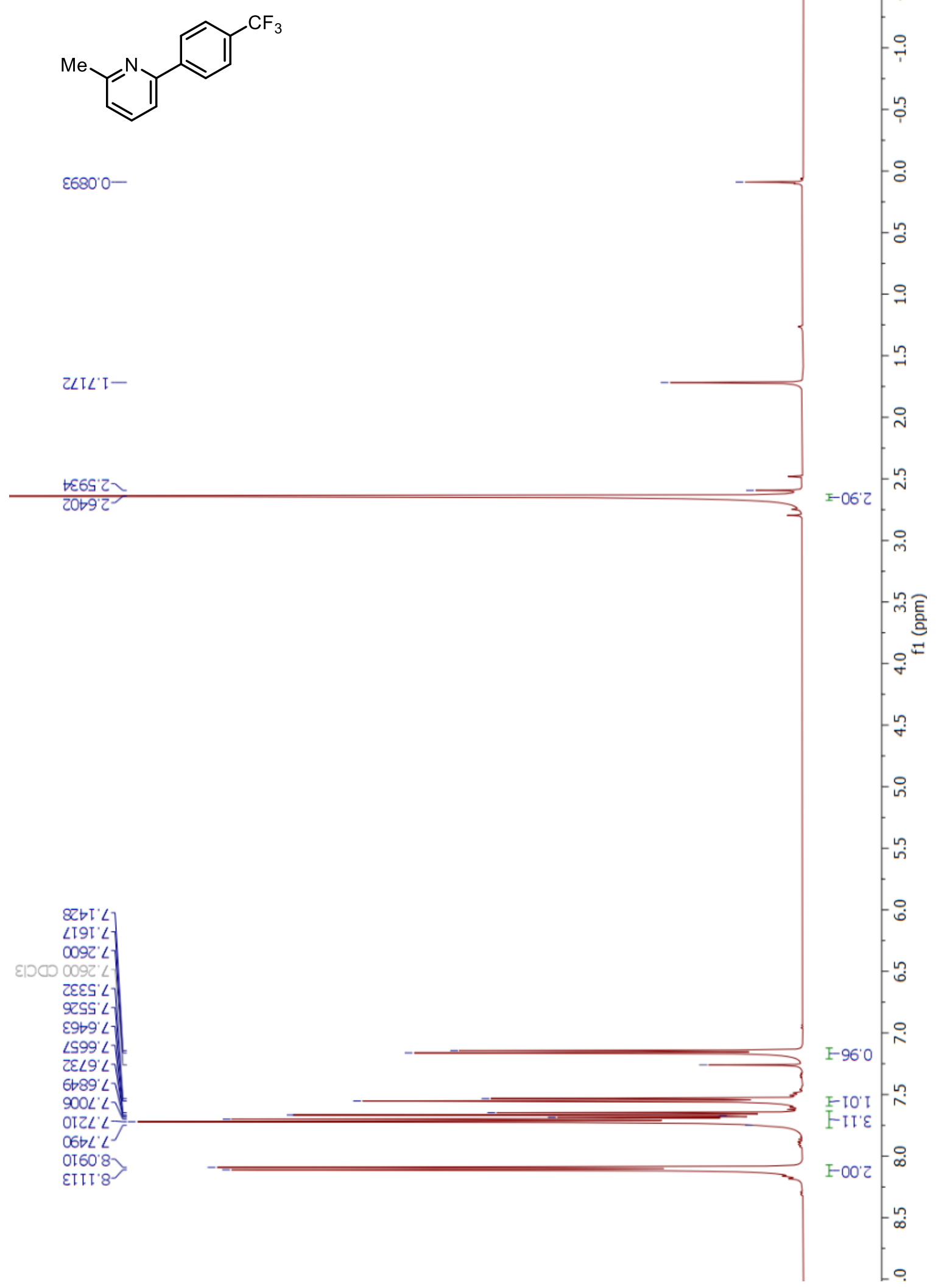

Figure S26. ${ }^{1} \mathrm{H}$ NMR (400 MHz) in $\mathrm{CDCl}_{3} 7 \mathrm{~d}$ 

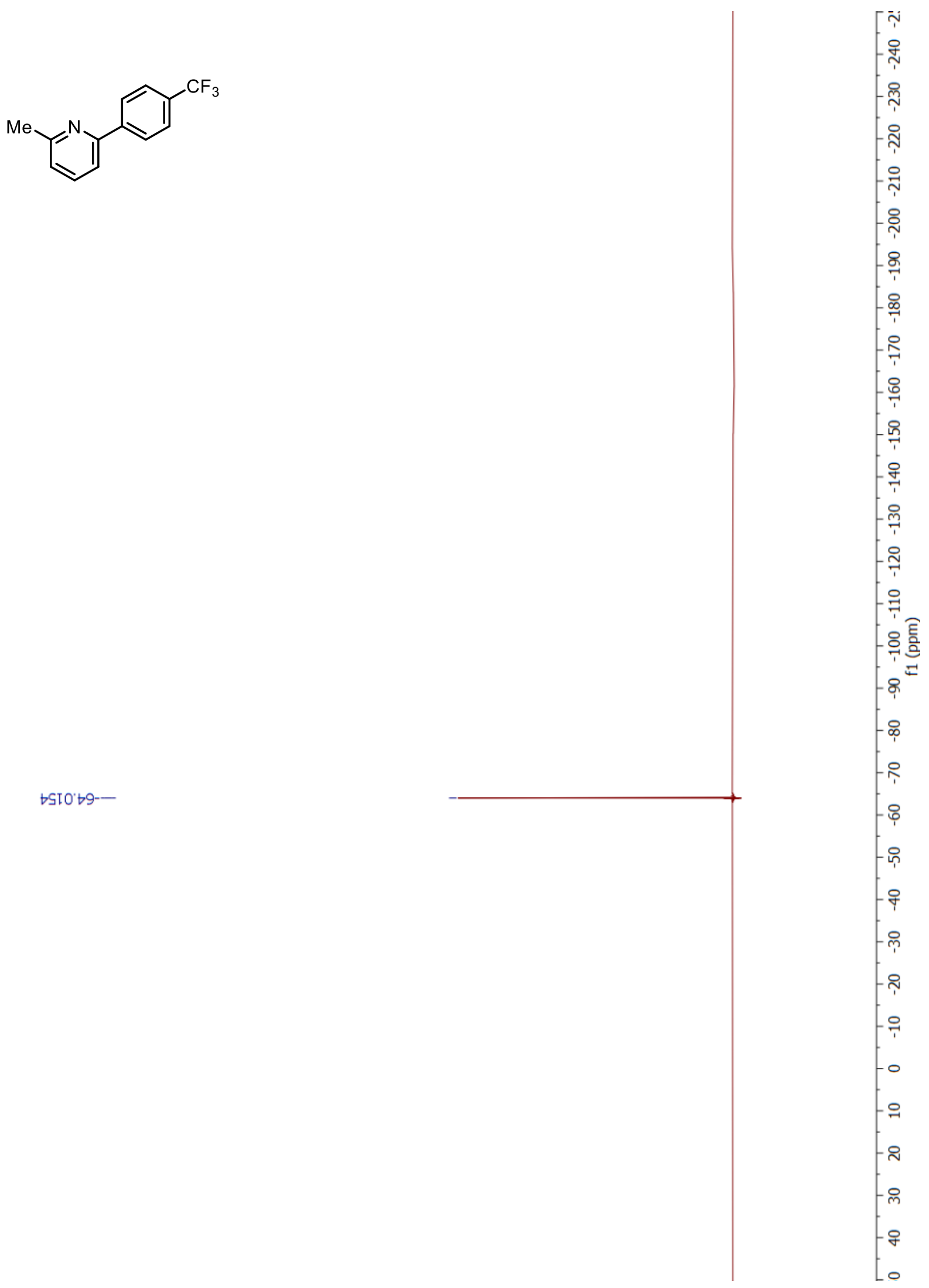

Figure S27. ${ }^{19}$ F NMR (376 MHz) in $\mathrm{CDCl}_{3} 7 \mathrm{~d}$ 


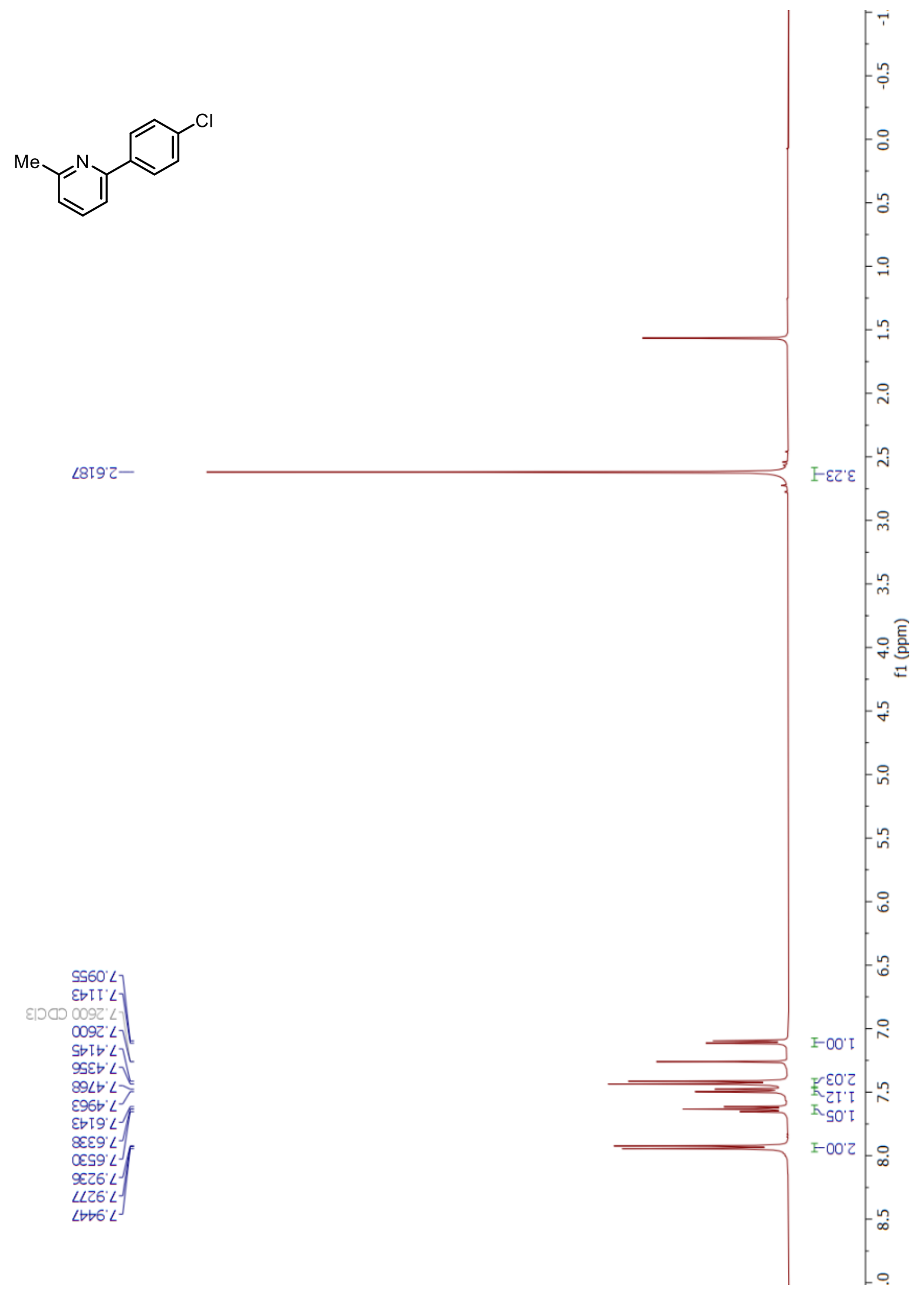

Figure S28. ${ }^{1} \mathrm{H}$ NMR (400 MHz) in $\mathrm{CDCl}_{3} 7 \mathrm{e}$ 


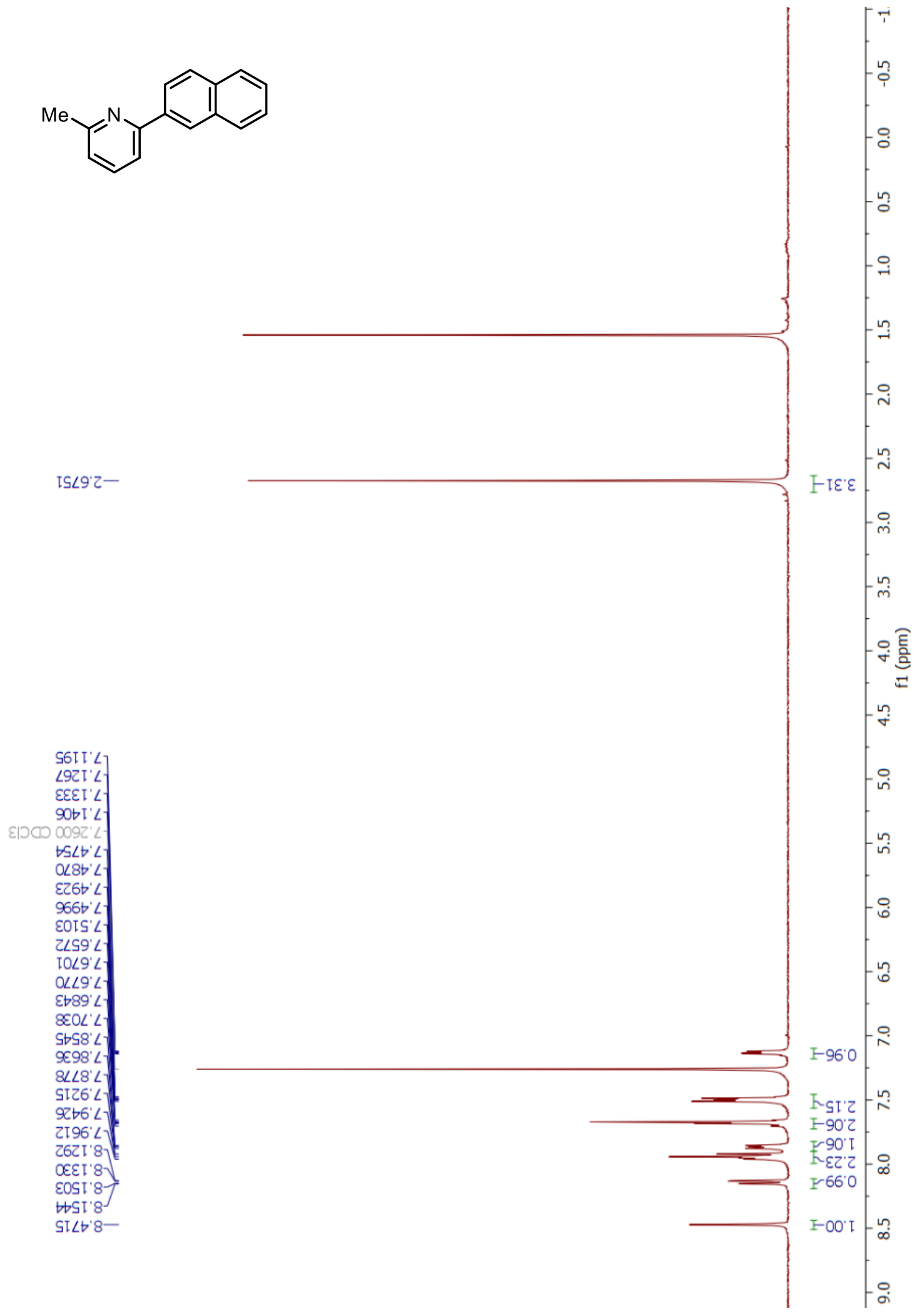

Figure S29. ${ }^{1} \mathrm{H}$ NMR (400 MHz) in $\mathrm{CDCl}_{3} 7 \mathrm{f}$ 

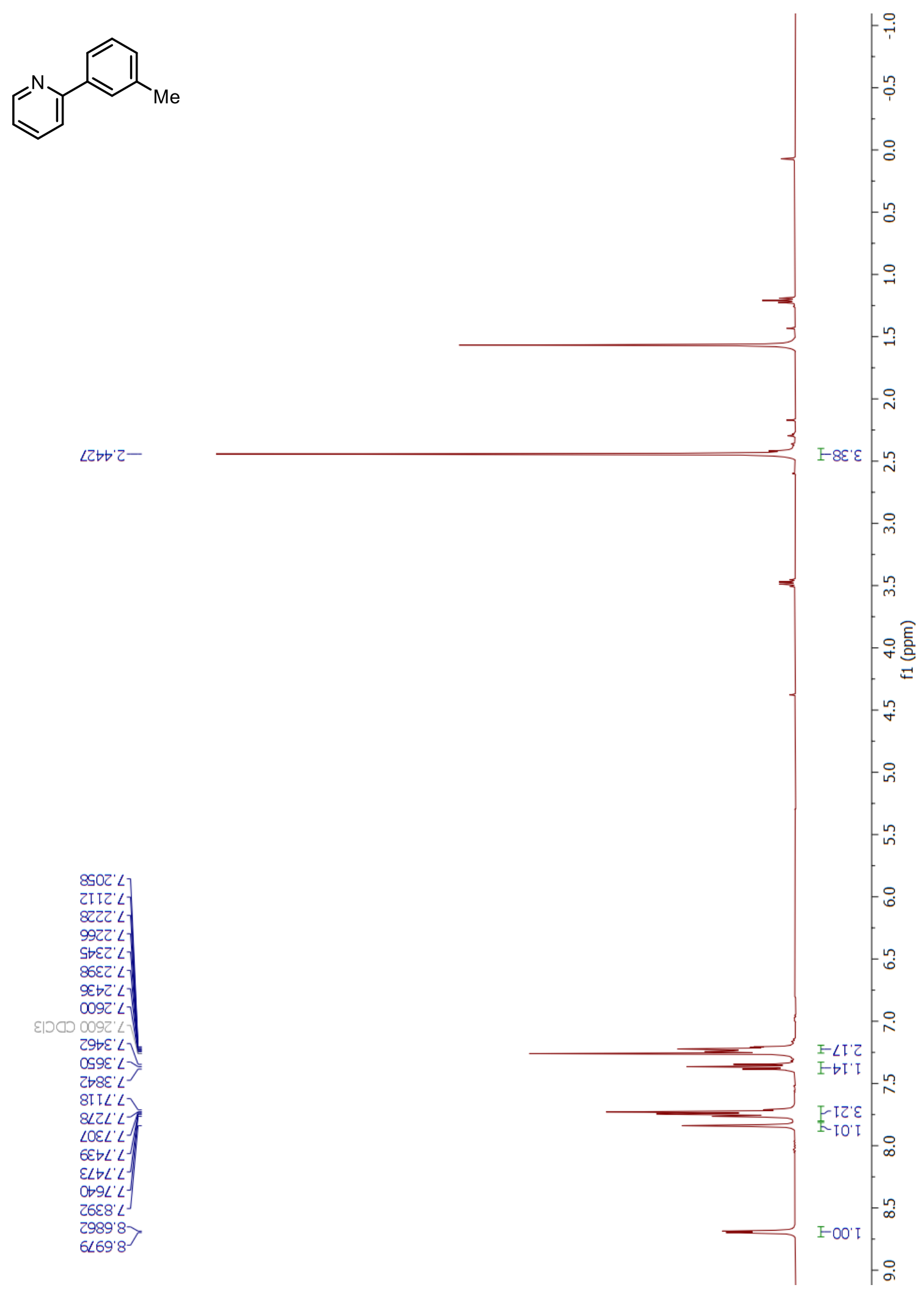

Figure S30. ${ }^{1} \mathrm{H}$ NMR (400 MHz) in $\mathrm{CDCl}_{3} 9 \mathrm{~b}$ 


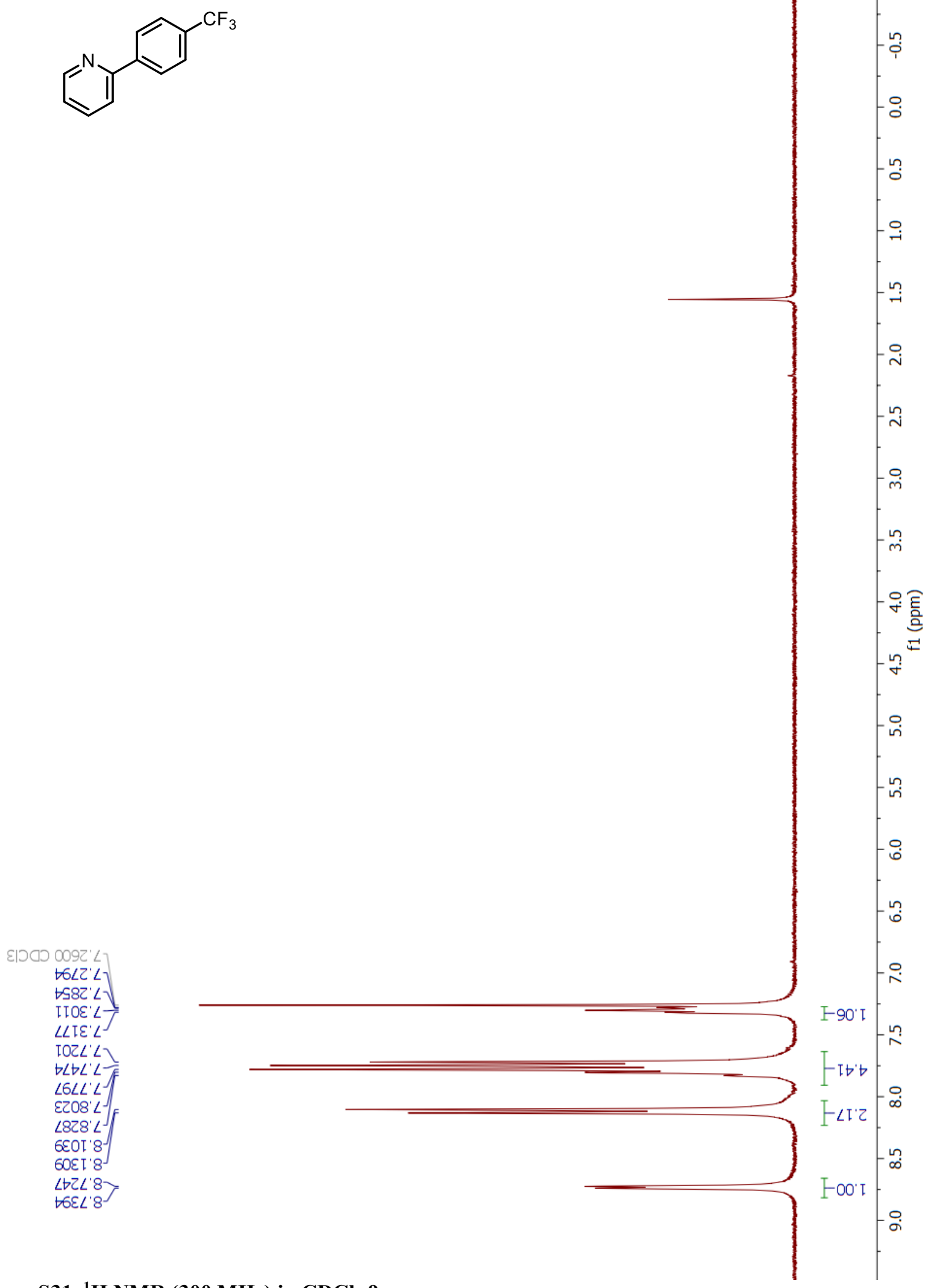

Figure S31. ${ }^{1} \mathrm{H}$ NMR (300 MHz) in $\mathrm{CDCl}_{3} 9 \mathrm{c}$ 

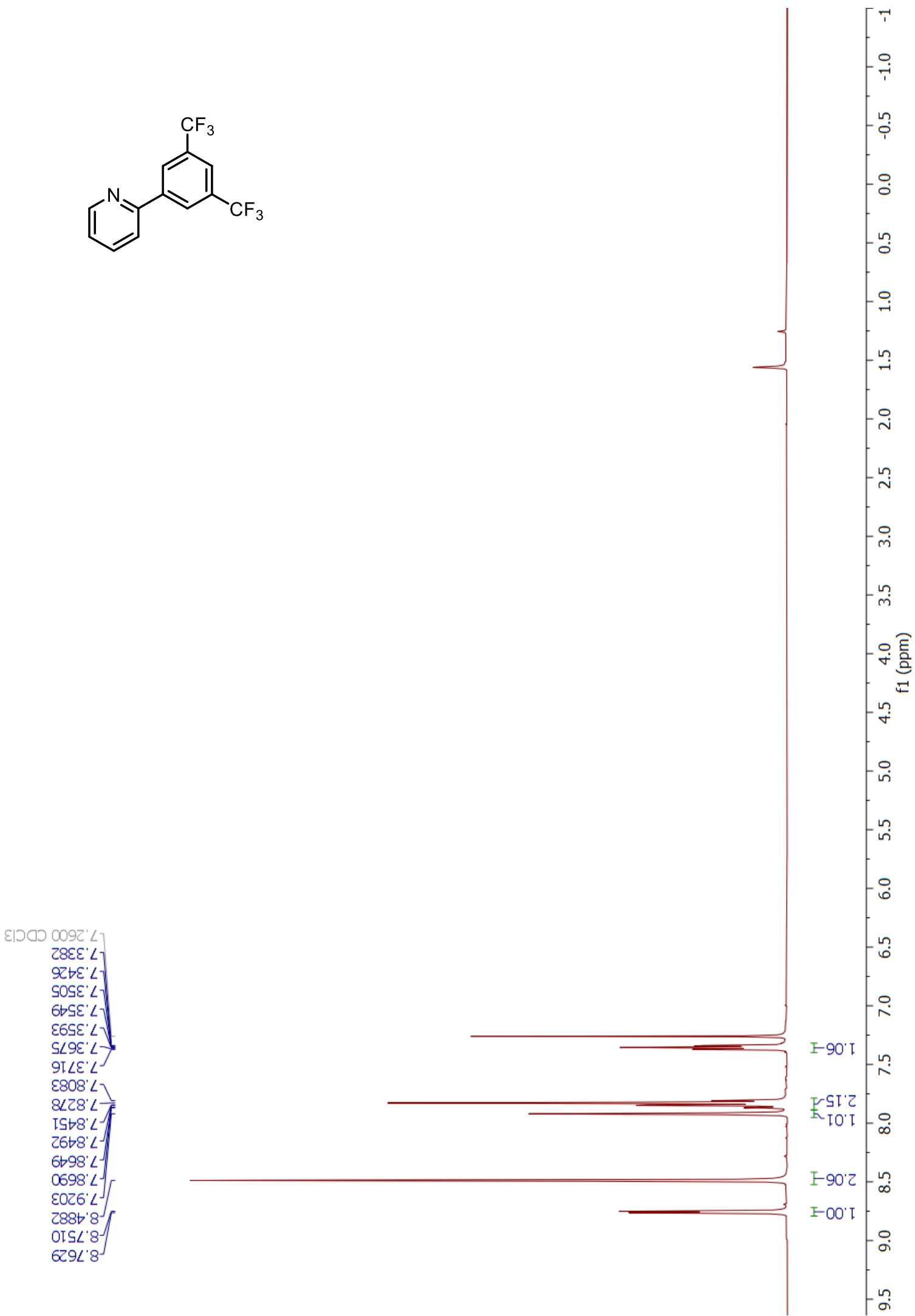

Figure S32. ${ }^{1} \mathrm{H}$ NMR (400 MHz) in $\mathrm{CDCl}_{3}$ 9d 

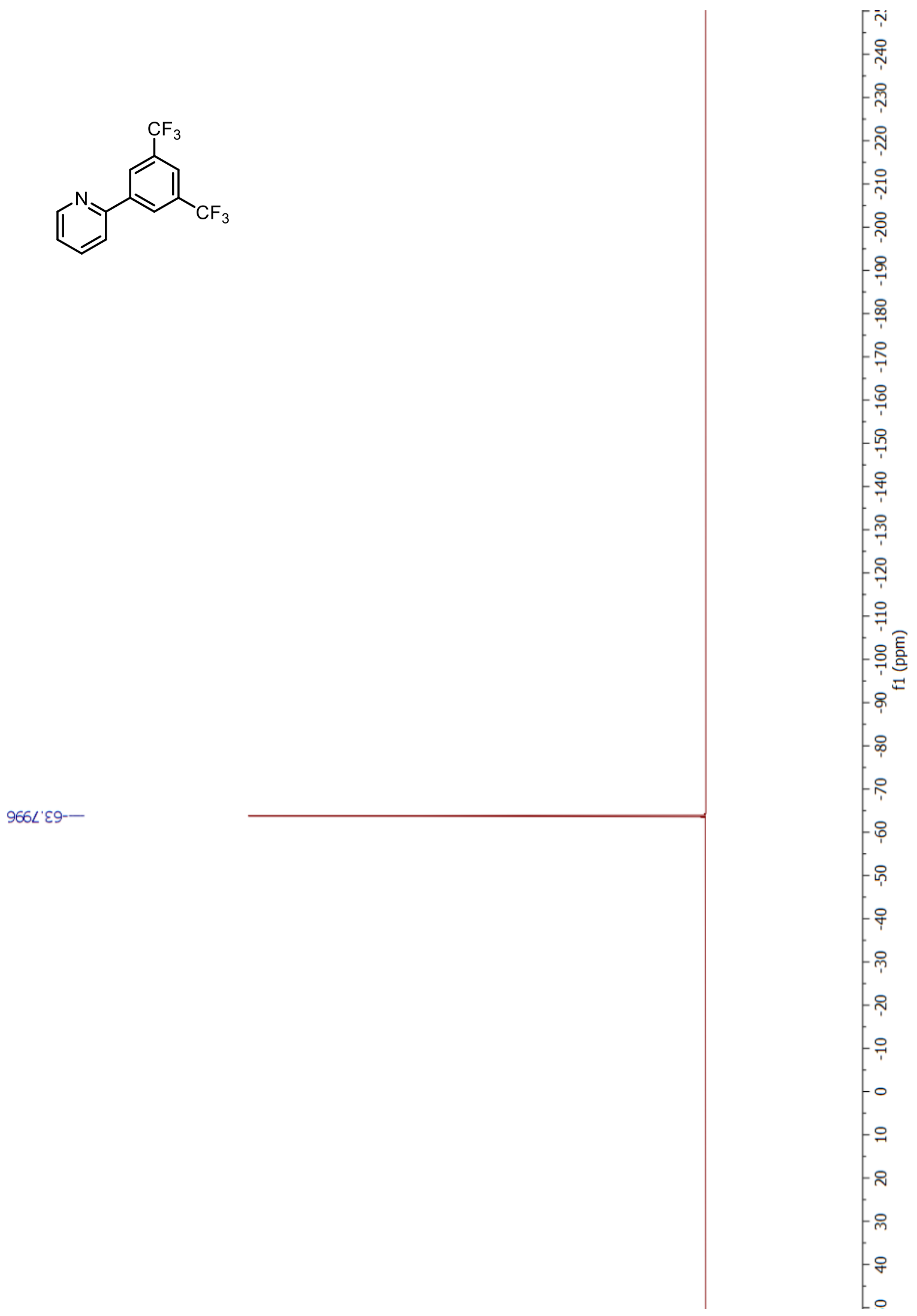

Figure S33. ${ }^{19} \mathrm{~F}$ NMR (376 MHz) in $\mathrm{CDCl}_{3}$ 9d 


\section{Spectra of Piperidine Products}
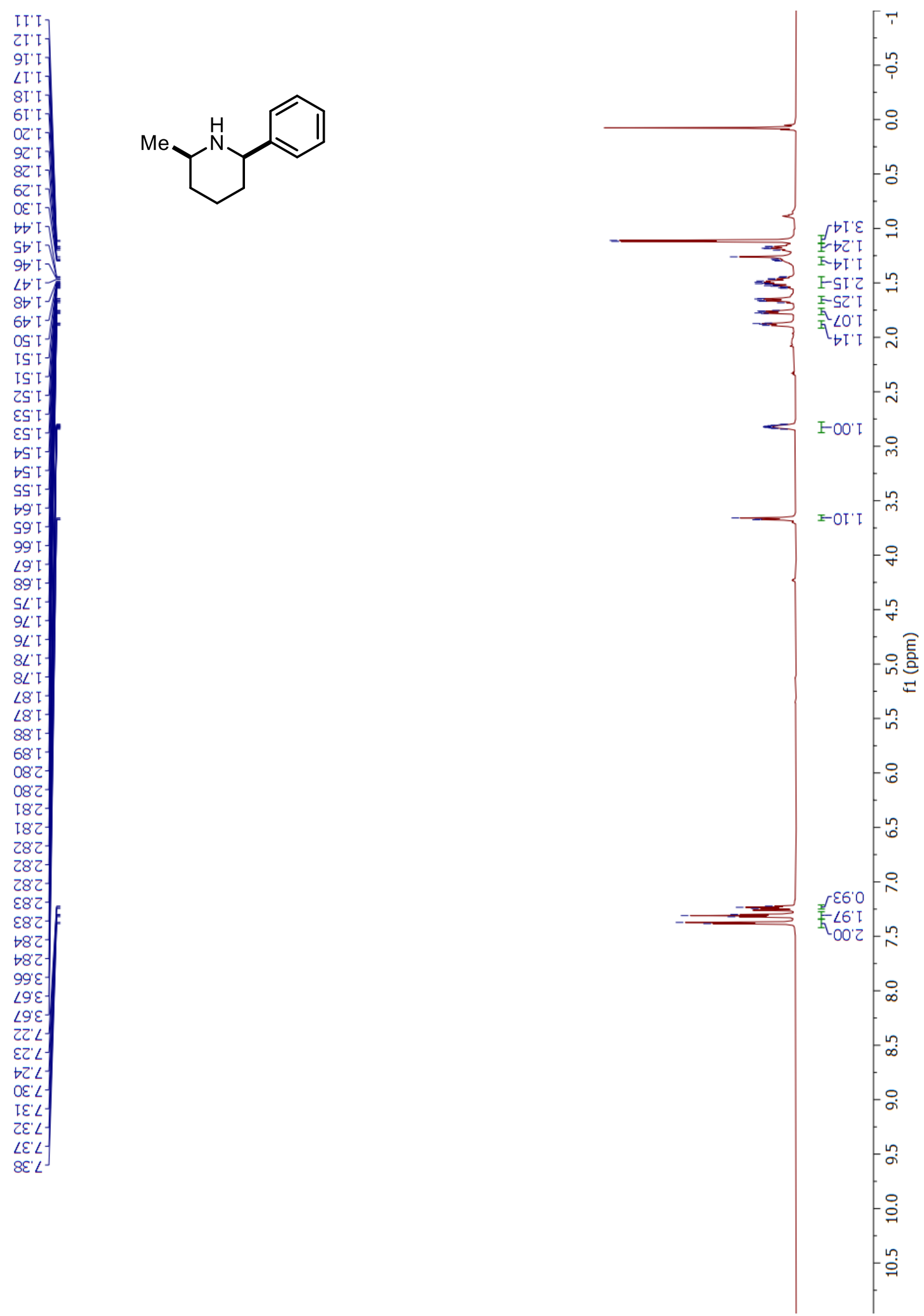

Figure S34. ${ }^{1} \mathrm{H}$ NMR (700 MHz) in $\mathrm{CDCl}_{3} 8 \mathrm{a}$ 


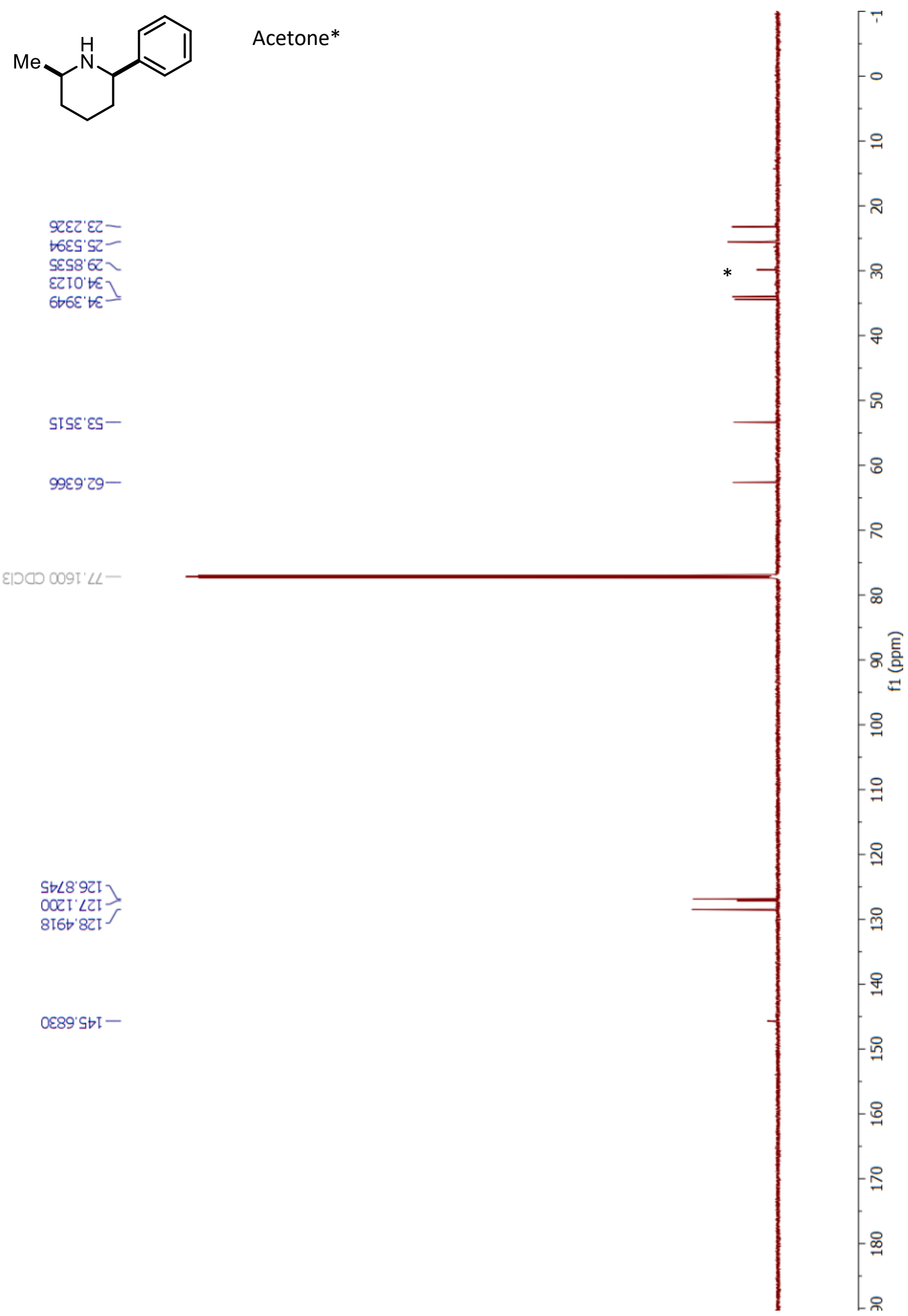

Figure S35. ${ }^{13} \mathrm{C}\left\{{ }^{1} \mathrm{H}\right\}$ NMR (176 MHz) in $\mathrm{CDCl}_{3} 8 \mathrm{a}$ 


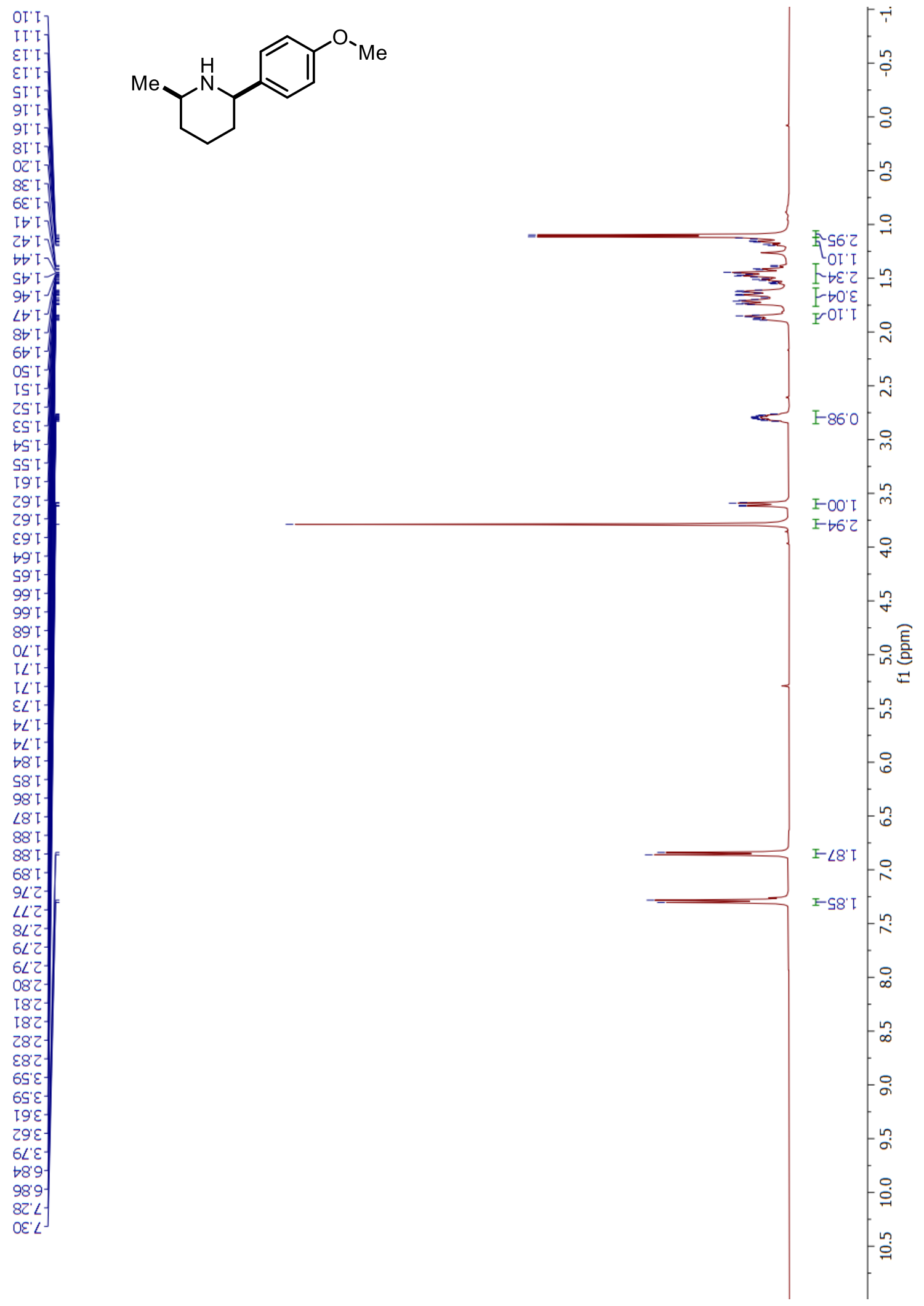

Figure S36. ${ }^{1} \mathrm{H}$ NMR (400 MHz) in $\mathrm{CDCl}_{3} 8 \mathrm{~b}$ 


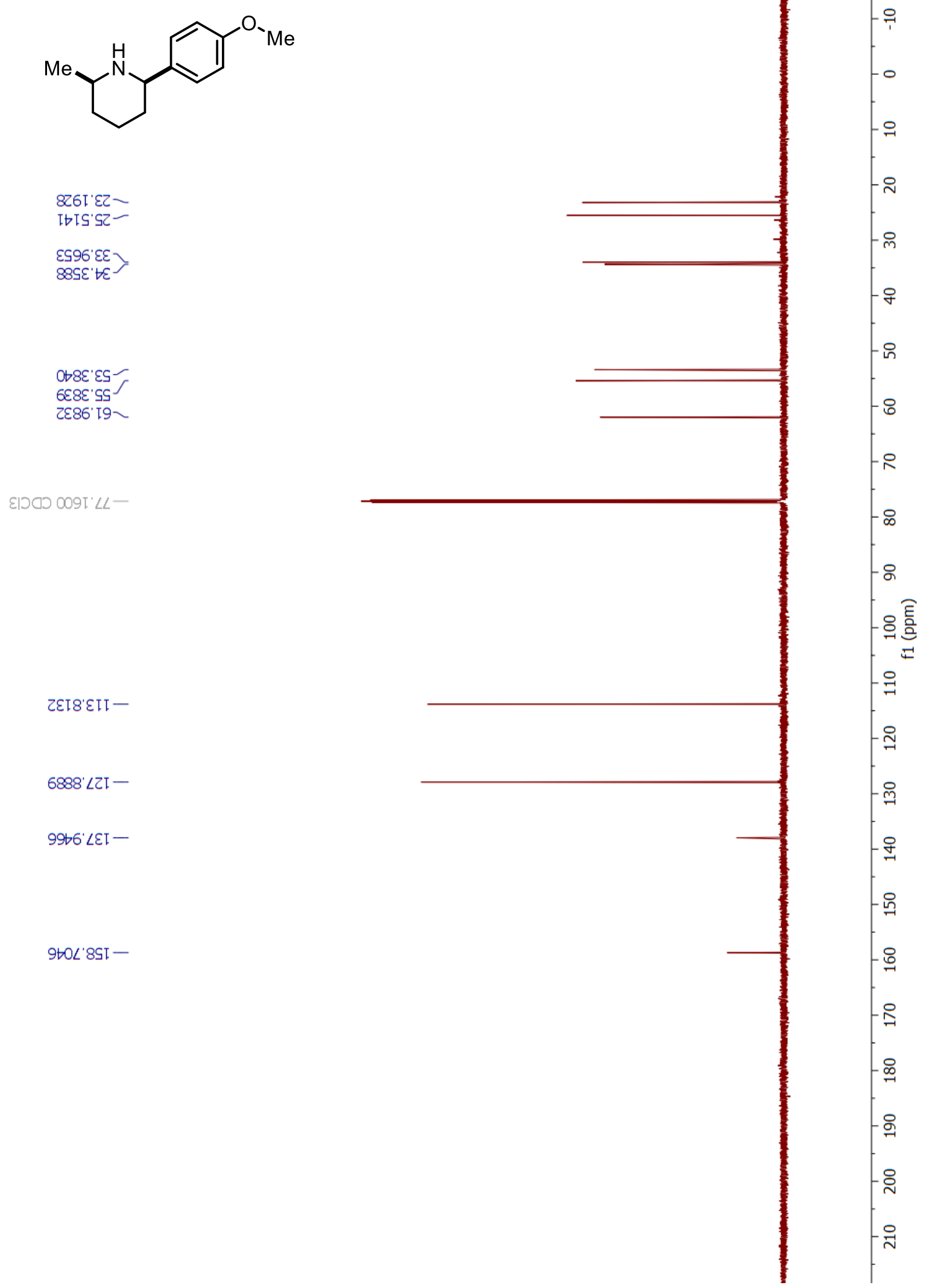

Figure S37. ${ }^{13} \mathrm{C}\left\{{ }^{1} \mathrm{H}\right\}$ NMR (176 MHz) in $\mathrm{CDCl}_{3} 8 \mathrm{~b}$ 


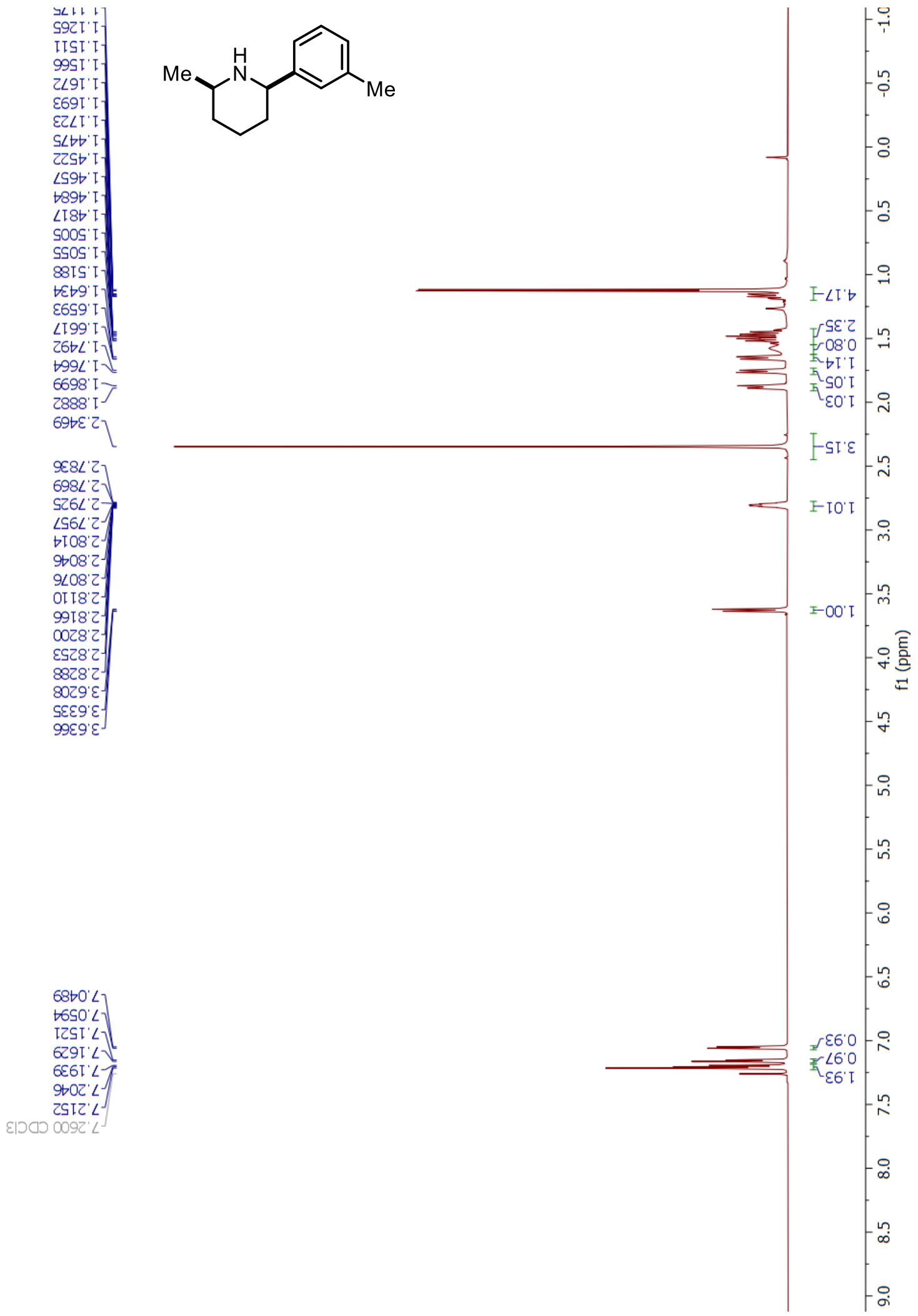

Figure S38. ${ }^{1} \mathrm{H}$ NMR (700MHz) in $\mathrm{CDCl}_{3} 8 \mathrm{c}$ 


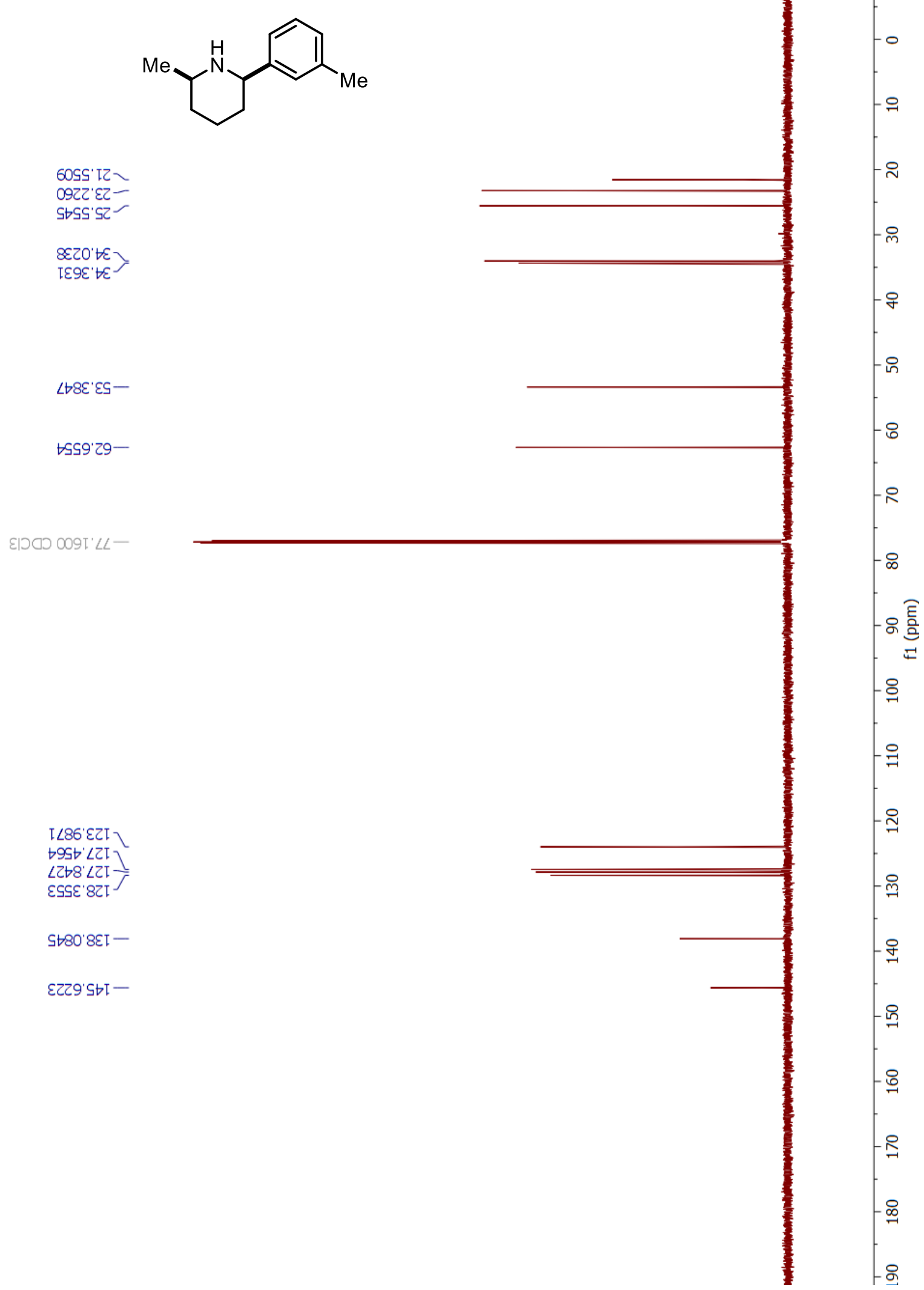

Figure S39. ${ }^{13} \mathrm{C}\left\{{ }^{1} \mathrm{H}\right\}$ NMR $(176 \mathrm{MHz})$ in $\mathrm{CDCl}_{3} 8 \mathrm{c}$ 


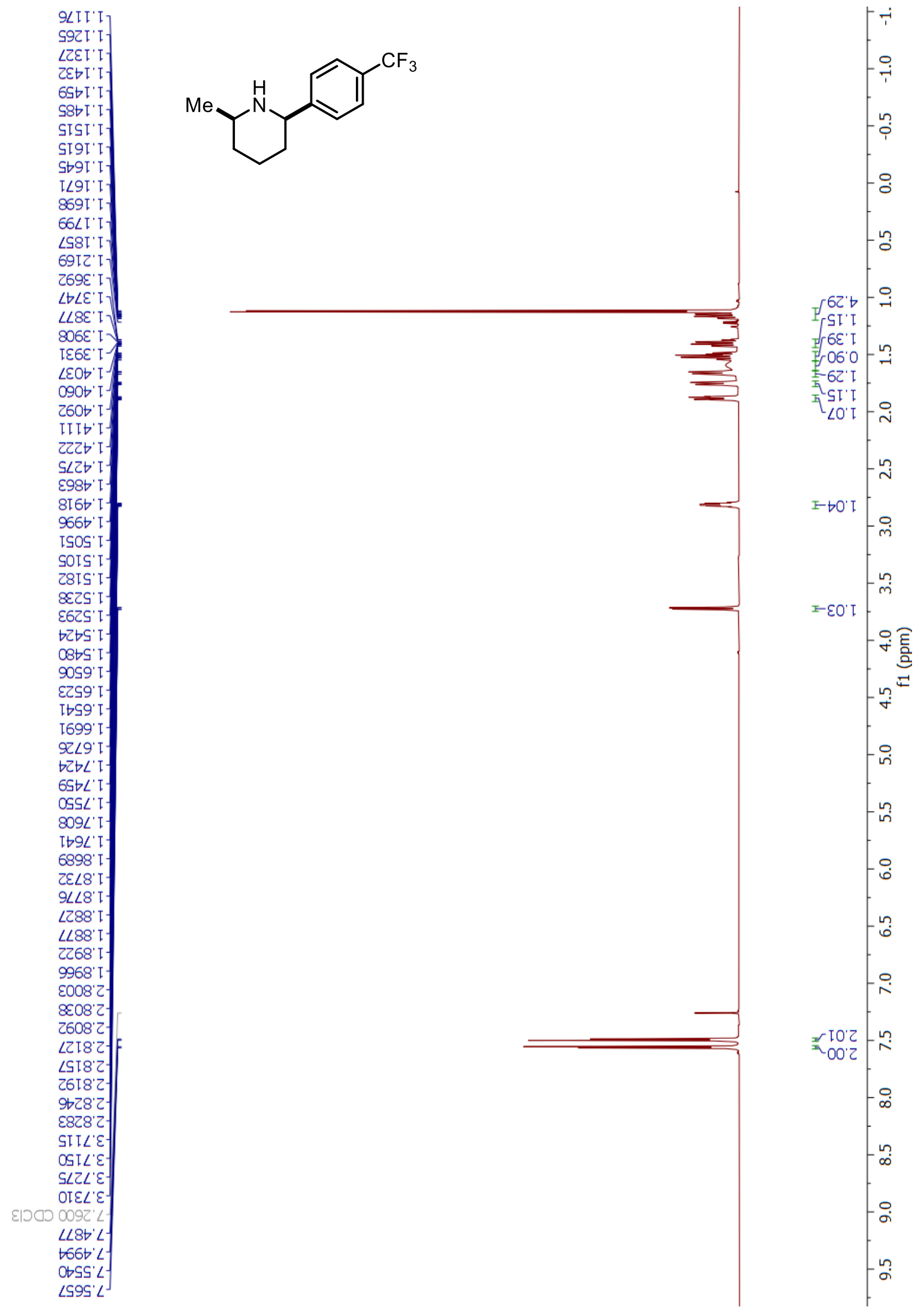

Figure S40. ${ }^{1} \mathrm{H}$ NMR $(700 \mathrm{MHz})$ in $\mathrm{CDCl}_{3} 8 \mathrm{~d}$ 


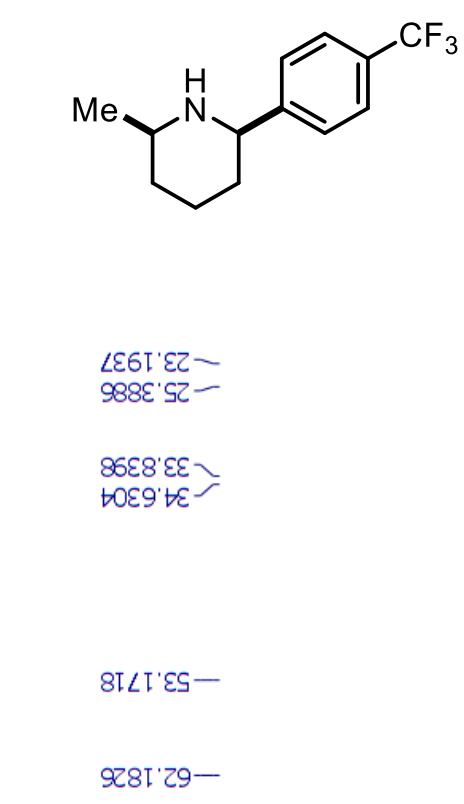

$\varepsilon \mid \supset 0 \supset$ $009 T^{\prime} \ll-$

9S60' ZZT

$\angle O b 9^{\prime} \varepsilon Z T$

ZZ8I'SZT

$669 \varepsilon$ 'SZT

9โ6ع'SZT

乙EโЬ'SZI

6tEt SZT

$\varepsilon \varepsilon Z L ' 9 Z T-$

$6 \rightarrow \angle T^{\prime} \angle Z T$

$\rightarrow 650$ '6ZT

66ع乙'6ZT

ObZt'6ZI

[809'62I]

$88 t L^{\prime} 6 t I-$

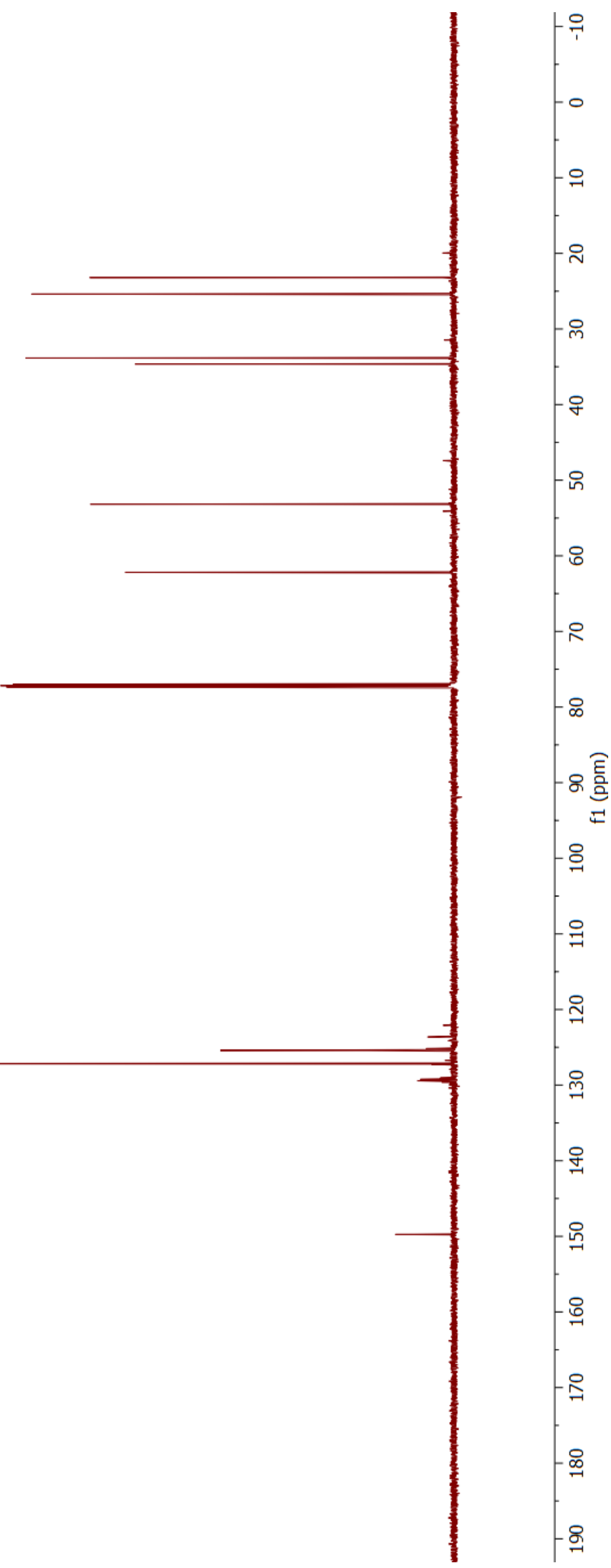

Figure S41. ${ }^{13} \mathrm{C}\left\{{ }^{1} \mathrm{H}\right\}$ NMR (176 MHz) in $\mathrm{CDCl}_{3} 8 \mathrm{~d}$ 


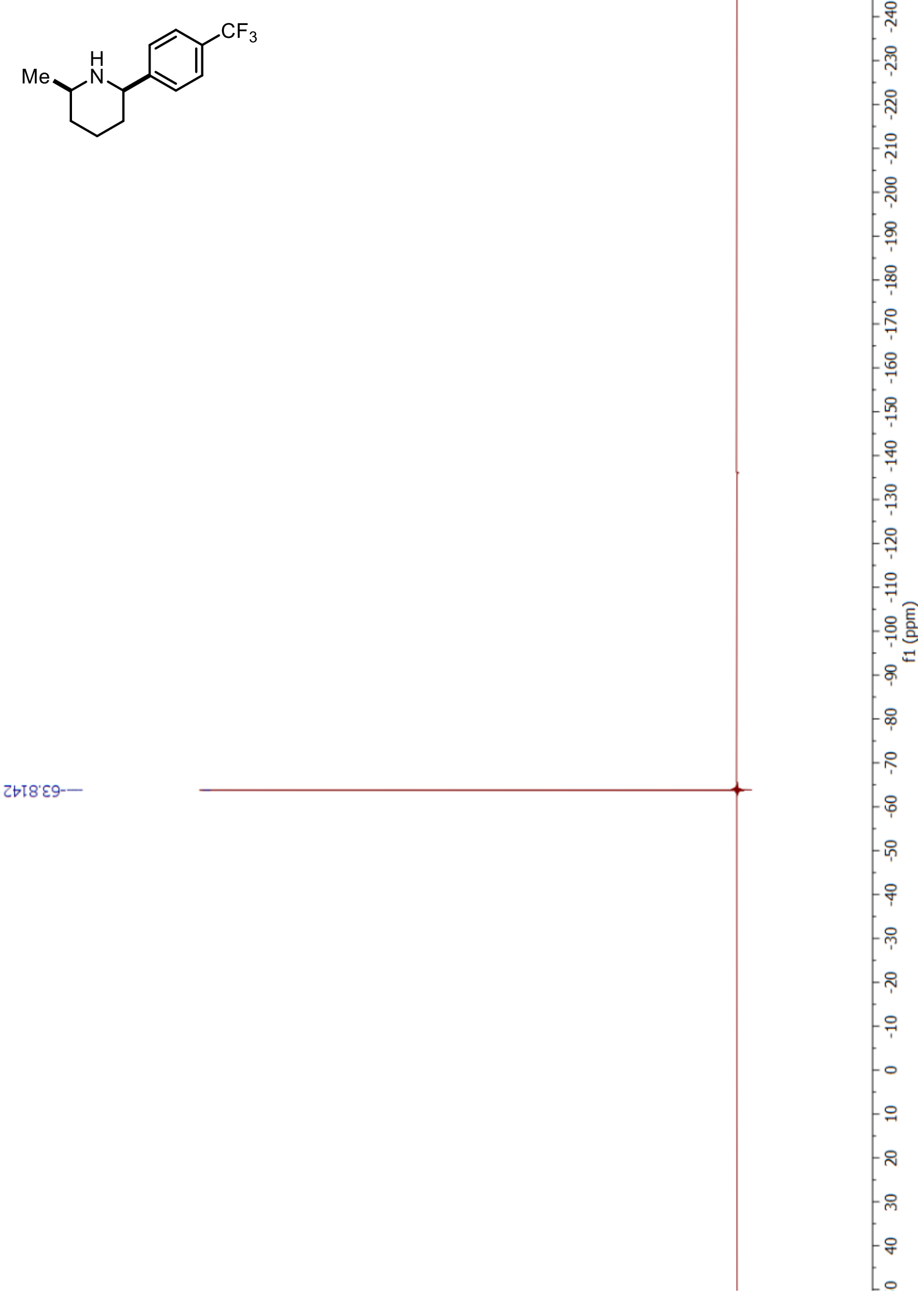

Figure S42. ${ }^{19} \mathrm{~F}$ NMR (376 MHz) in $\mathrm{CDCl}_{3} 8 \mathrm{~d}$ 


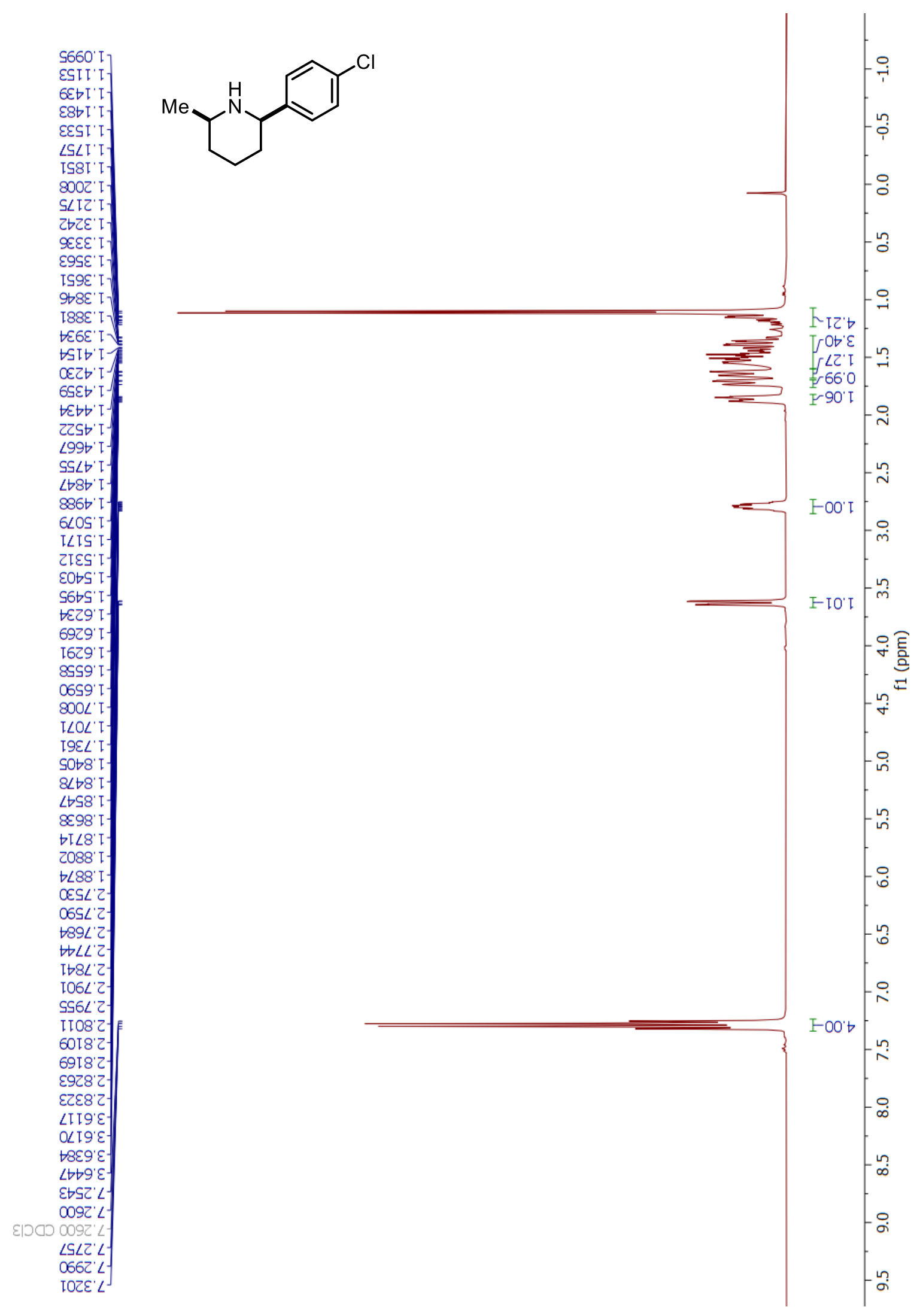

Figure S43. ${ }^{1} \mathrm{H}$ NMR (400 MHz) in $\mathrm{CDCl}_{3} 8 \mathrm{e}$ 


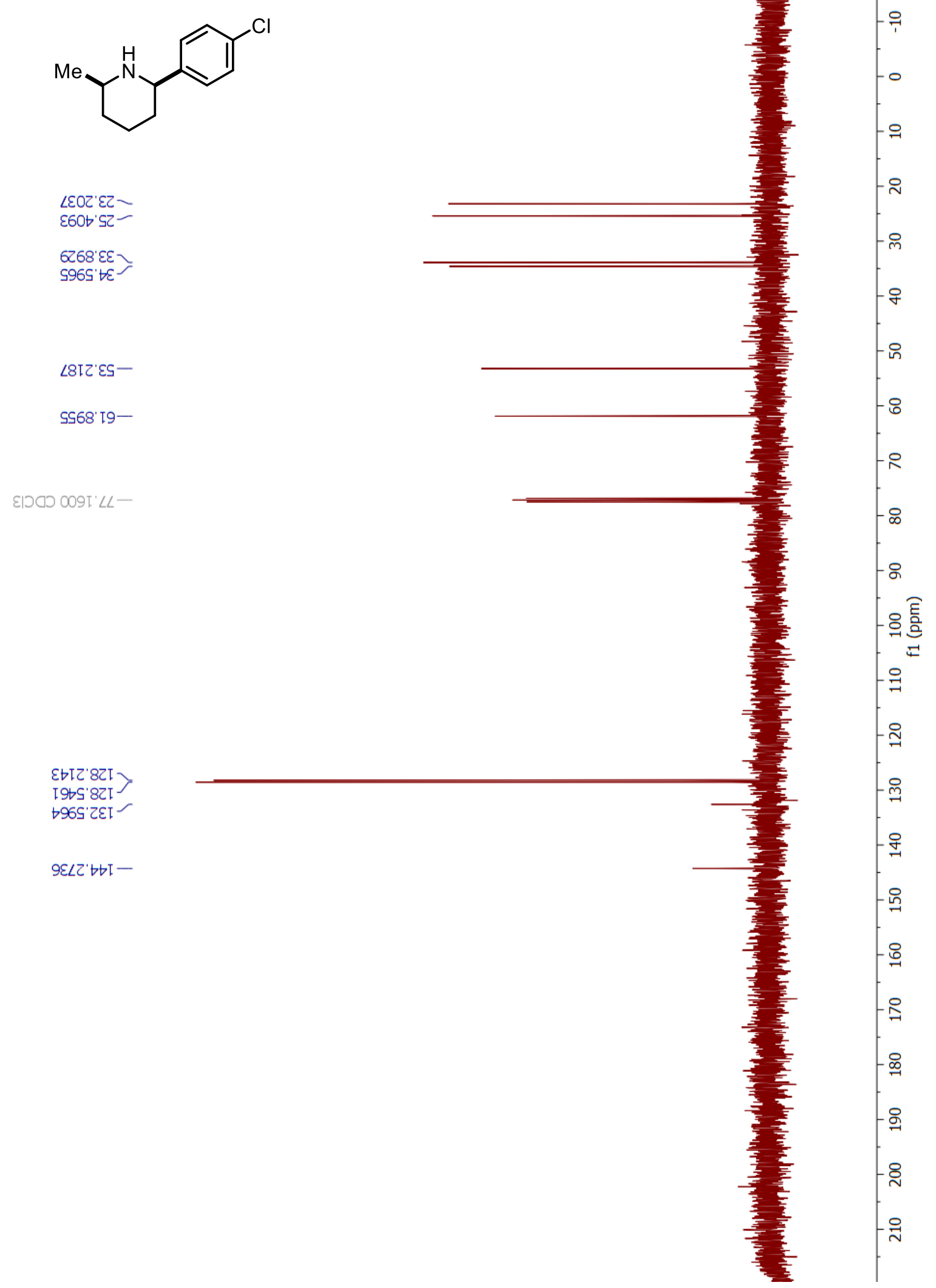

Figure S44. ${ }^{13} \mathrm{C}\left\{{ }^{1} \mathrm{H}\right\}$ NMR $(101 \mathrm{MHz})$ in $\mathrm{CDCl}_{3} 8 \mathrm{e}$ 

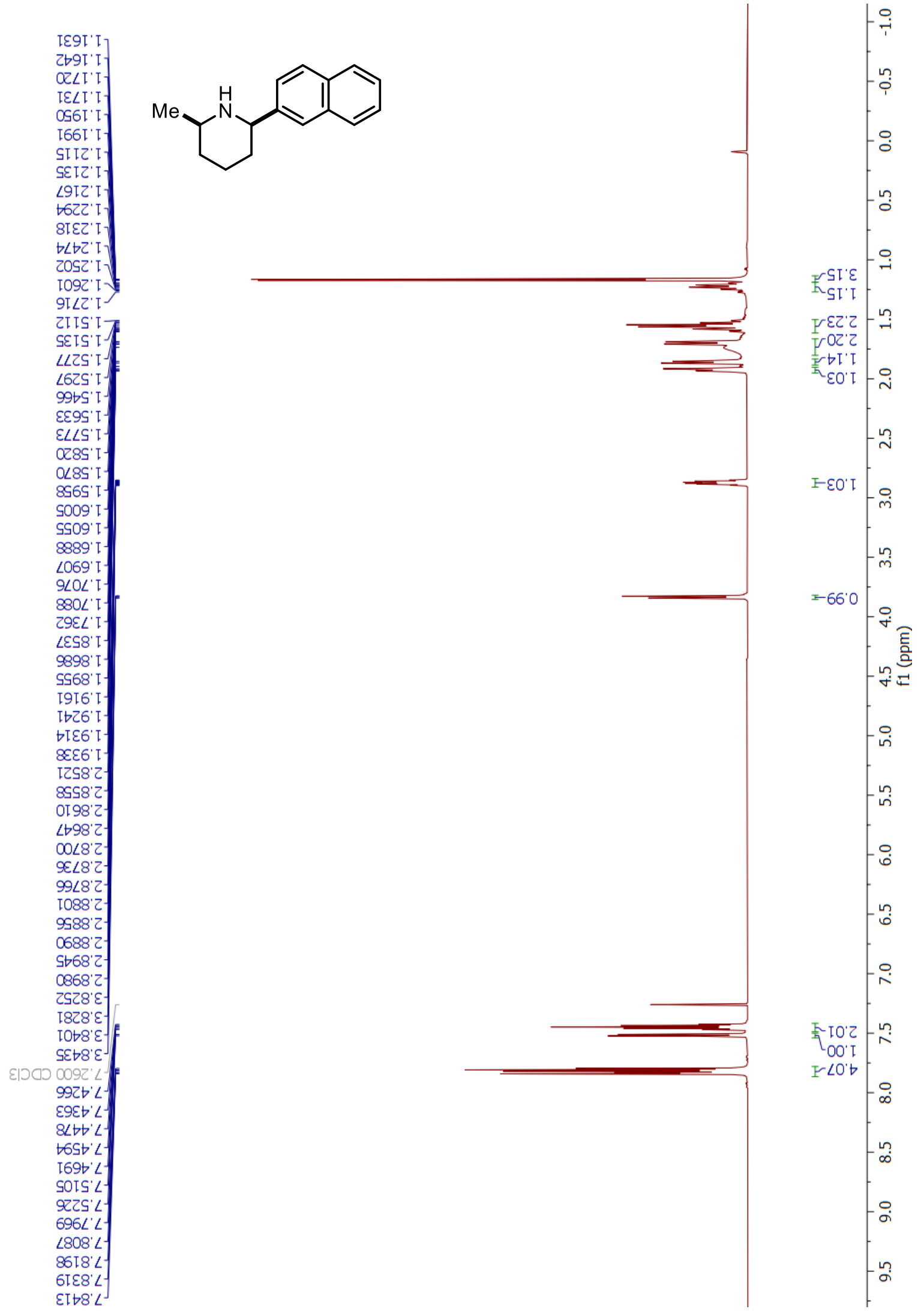

Figure S45. ${ }^{1} \mathrm{H}$ NMR (700MHz) in $\mathrm{CDCl}_{3} 8 \mathrm{f}$ 


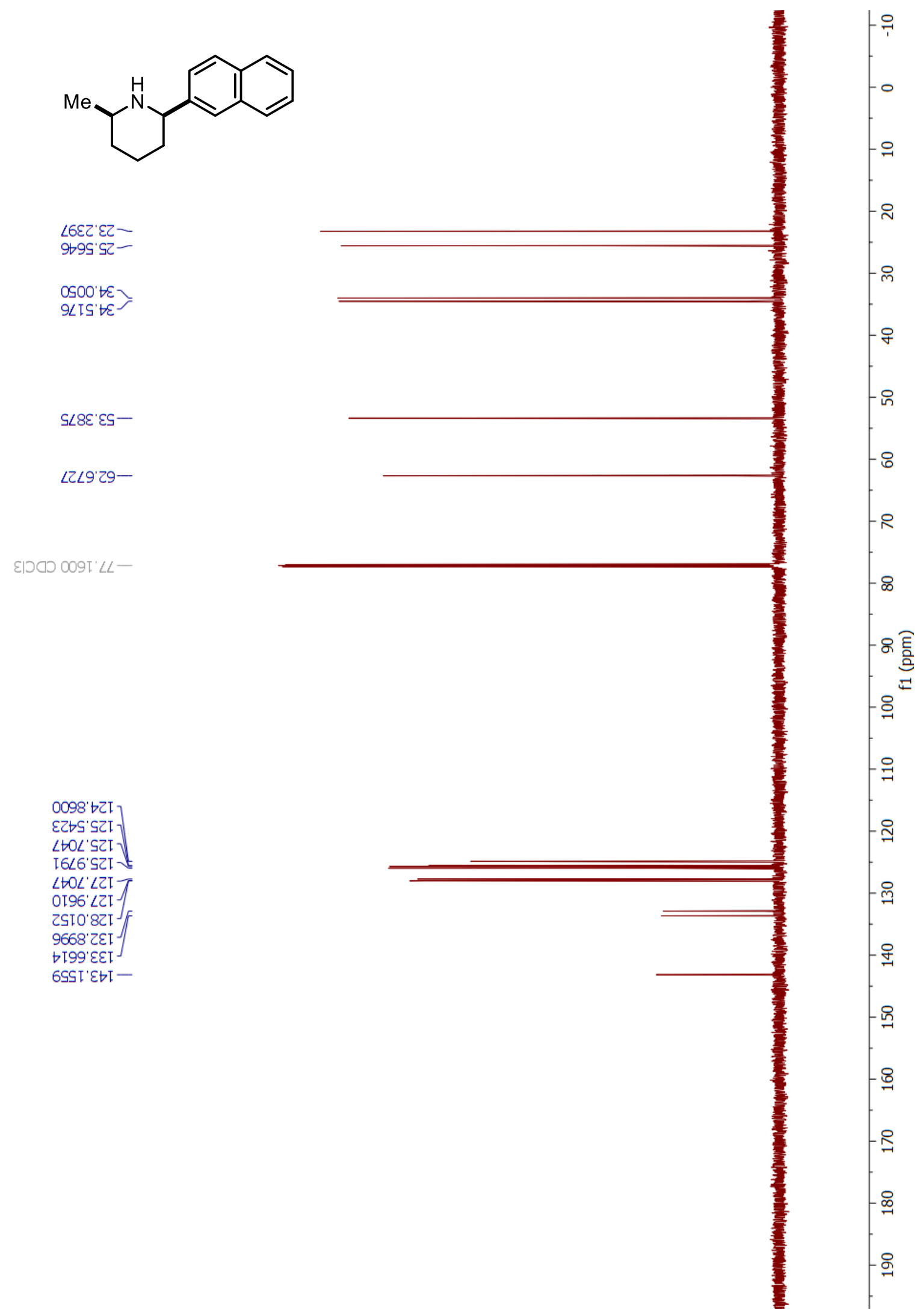

Figure S46. ${ }^{13} \mathrm{C}\left\{{ }^{1} \mathrm{H}\right\}$ NMR $(176 \mathrm{MHz})$ in $\mathrm{CDCl}_{3} 8 f$ 


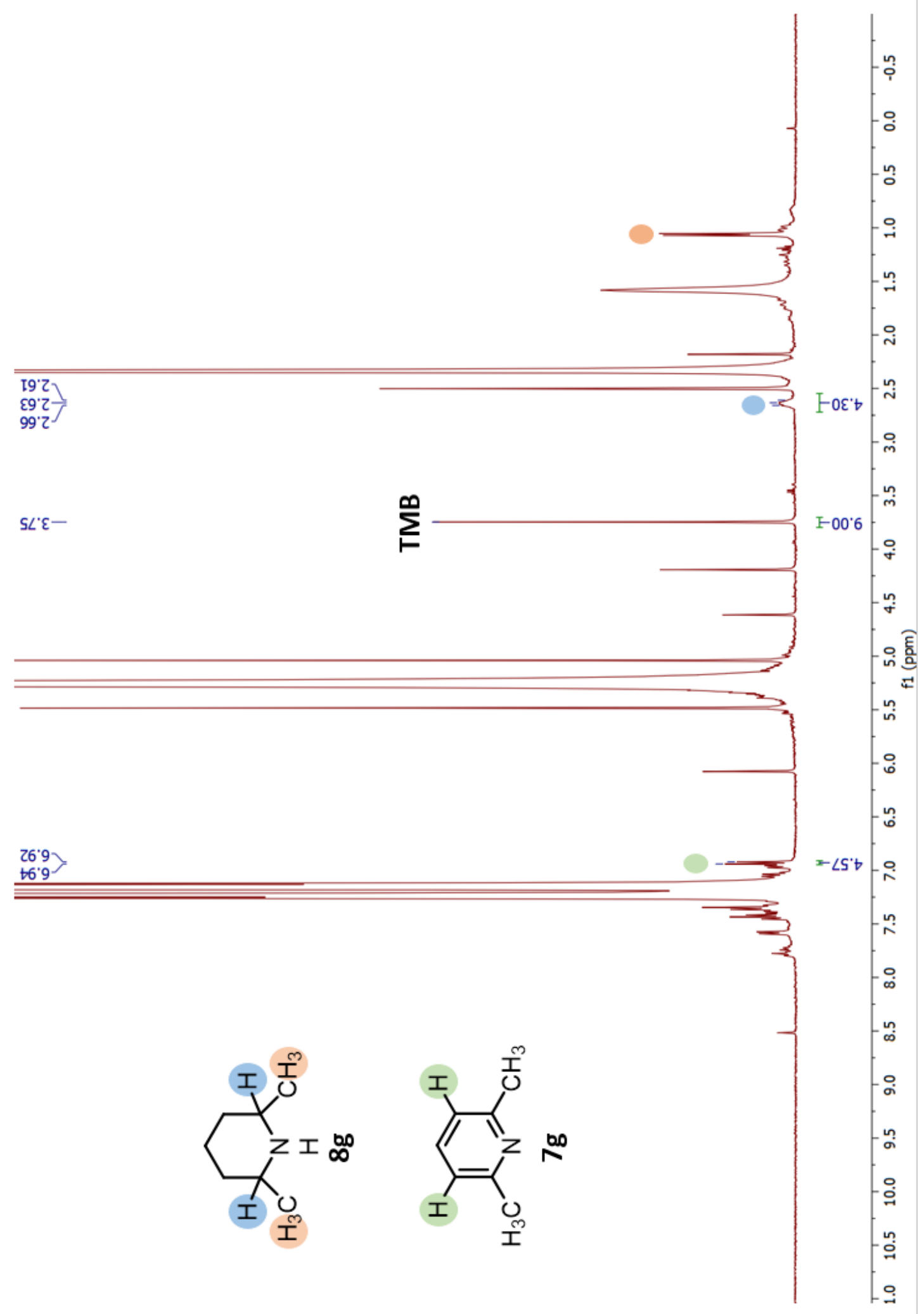


Figure S47. ${ }^{1} \mathrm{H}$ NMR (400MHz) in $\mathrm{CDCl}_{3} 8 \mathrm{~g}(\mathrm{TMB}=1,3,5$-trimethoxy benzene)

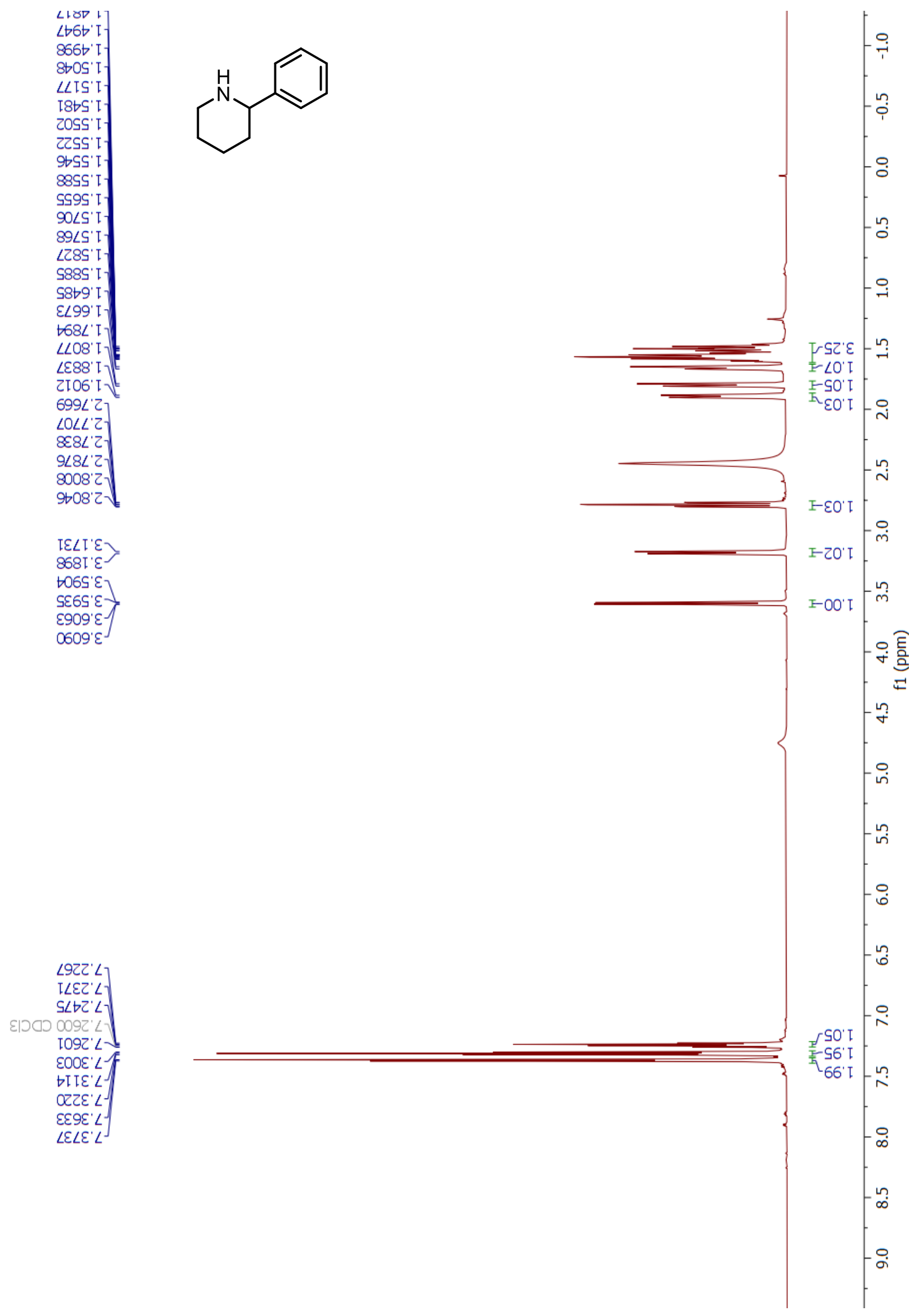


Figure S48. ${ }^{1} \mathrm{H}$ NMR $(700 \mathrm{MHz})$ in $\mathrm{CDCl}_{3} \mathbf{1 0 a}$

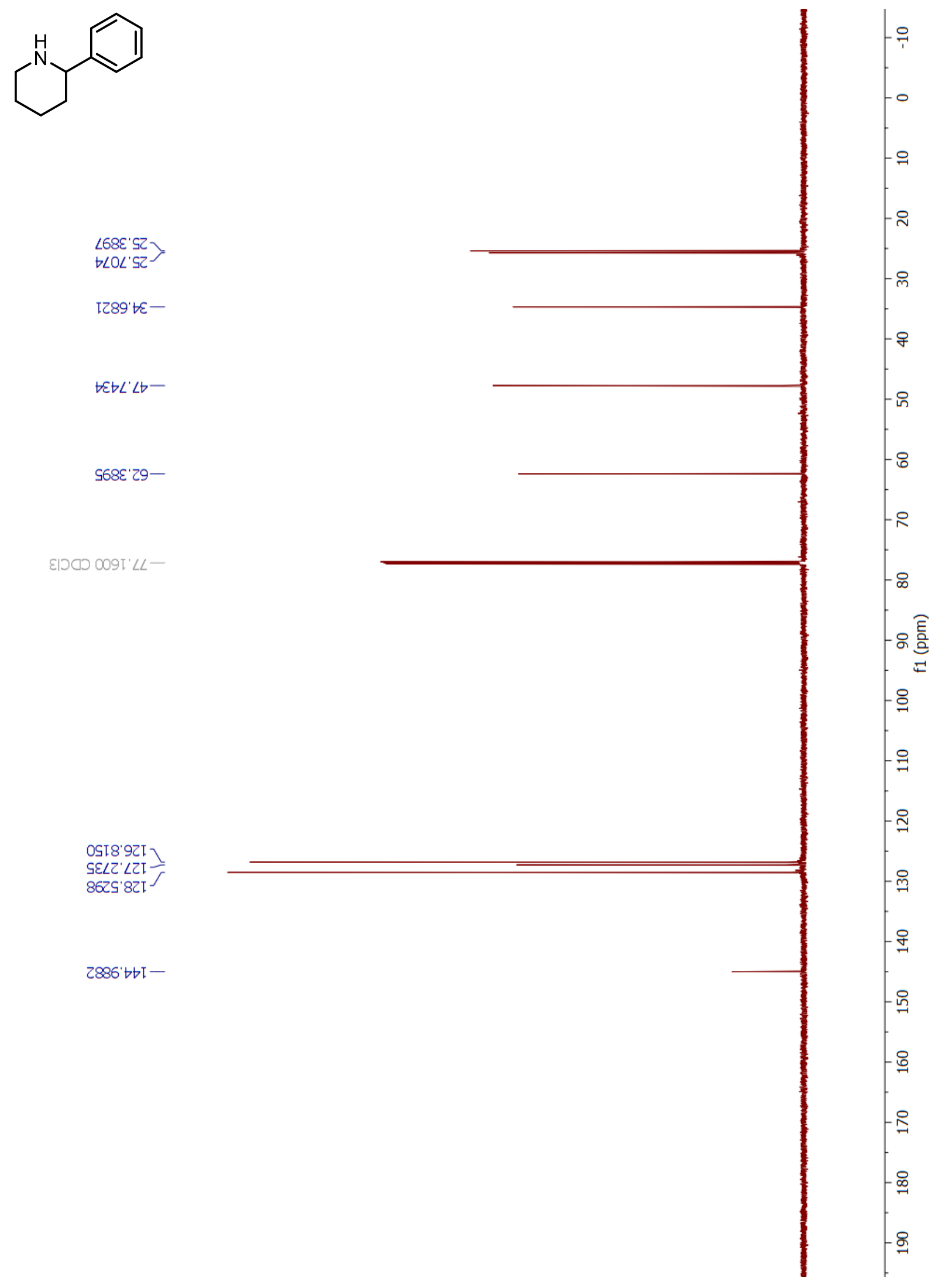


Figure S49. ${ }^{13} \mathrm{C}\left\{{ }^{1} \mathrm{H}\right\}$ NMR (176 MHz) in $\mathrm{CDCl}_{3}$ 10a
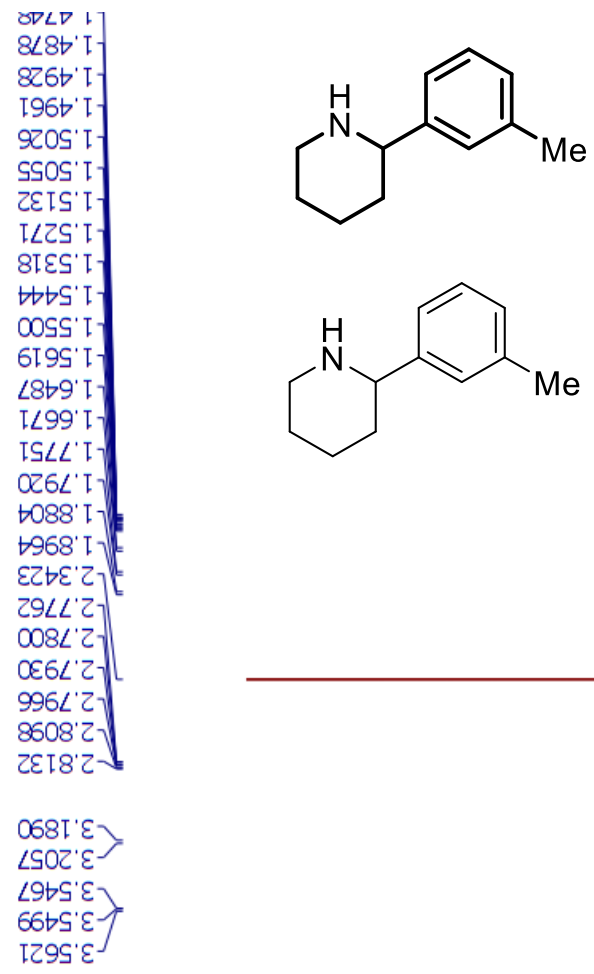

88b0' L

2650 ' $\angle$ ]

$\forall 9 \varepsilon T^{\prime}<\sqrt{ }$

$\tau \angle b T \angle V$

9I6I'LT

$\angle 2 O Z^{\prime} \angle$

$6 Z 1 Z^{\prime} L$
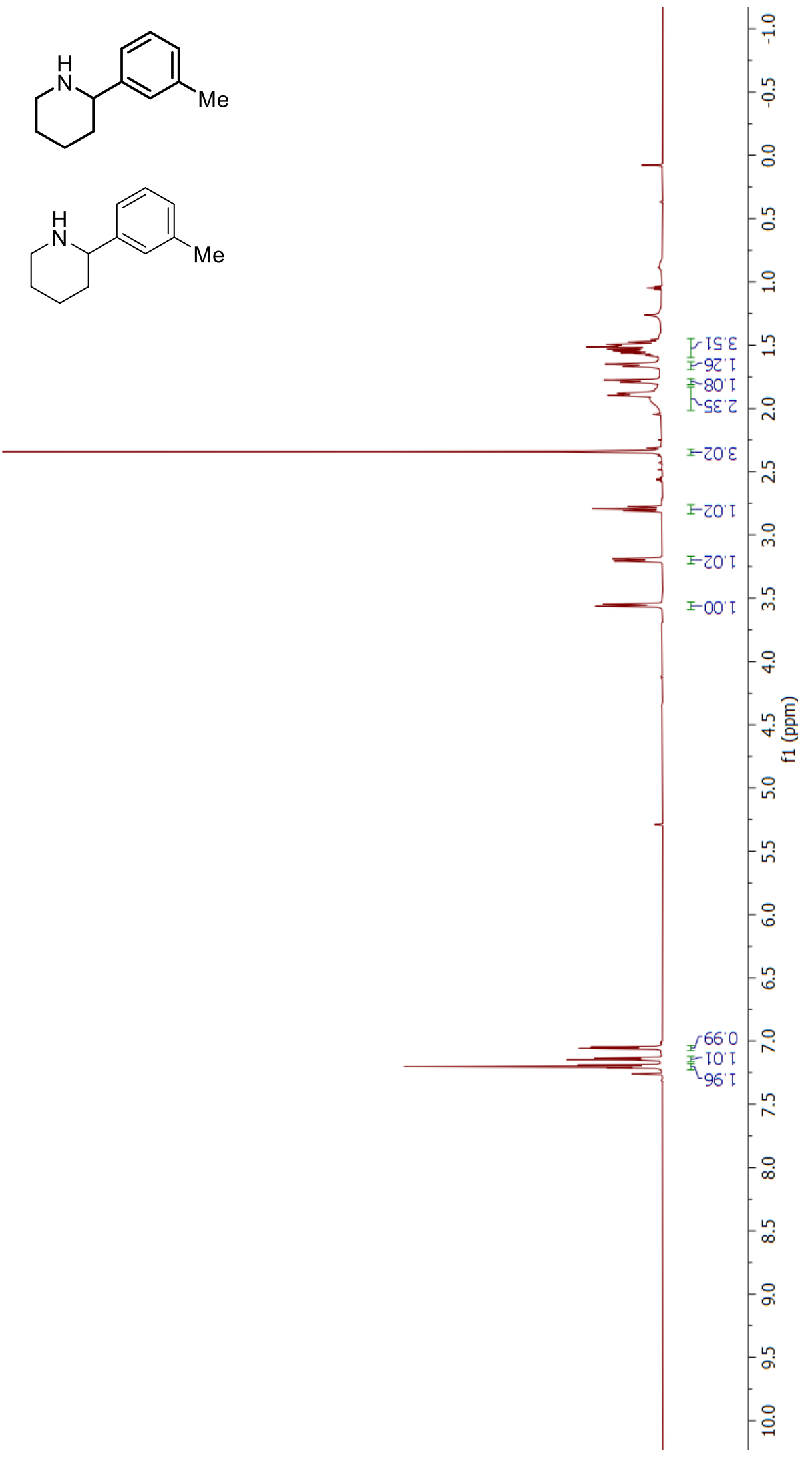
Figure S50. ${ }^{1} \mathrm{H}$ NMR $(700 \mathrm{MHz})$ in $\mathrm{CDCl}_{3}$ 10b

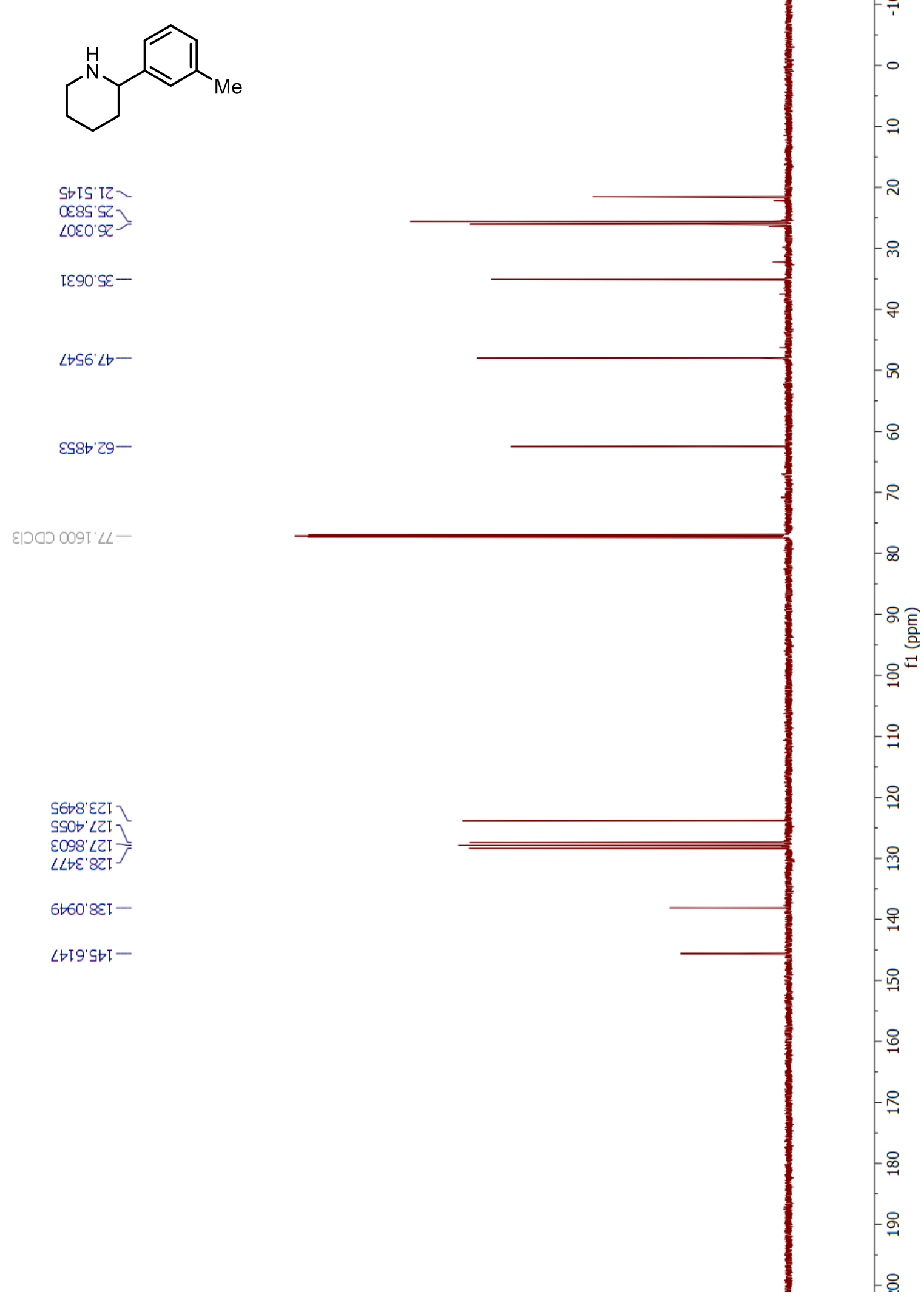


Figure S51. ${ }^{13} \mathrm{C}\left\{{ }^{1} \mathrm{H}\right\}$ NMR (176 MHz) in $\mathrm{CDCl}_{3} 10 \mathrm{~b}$
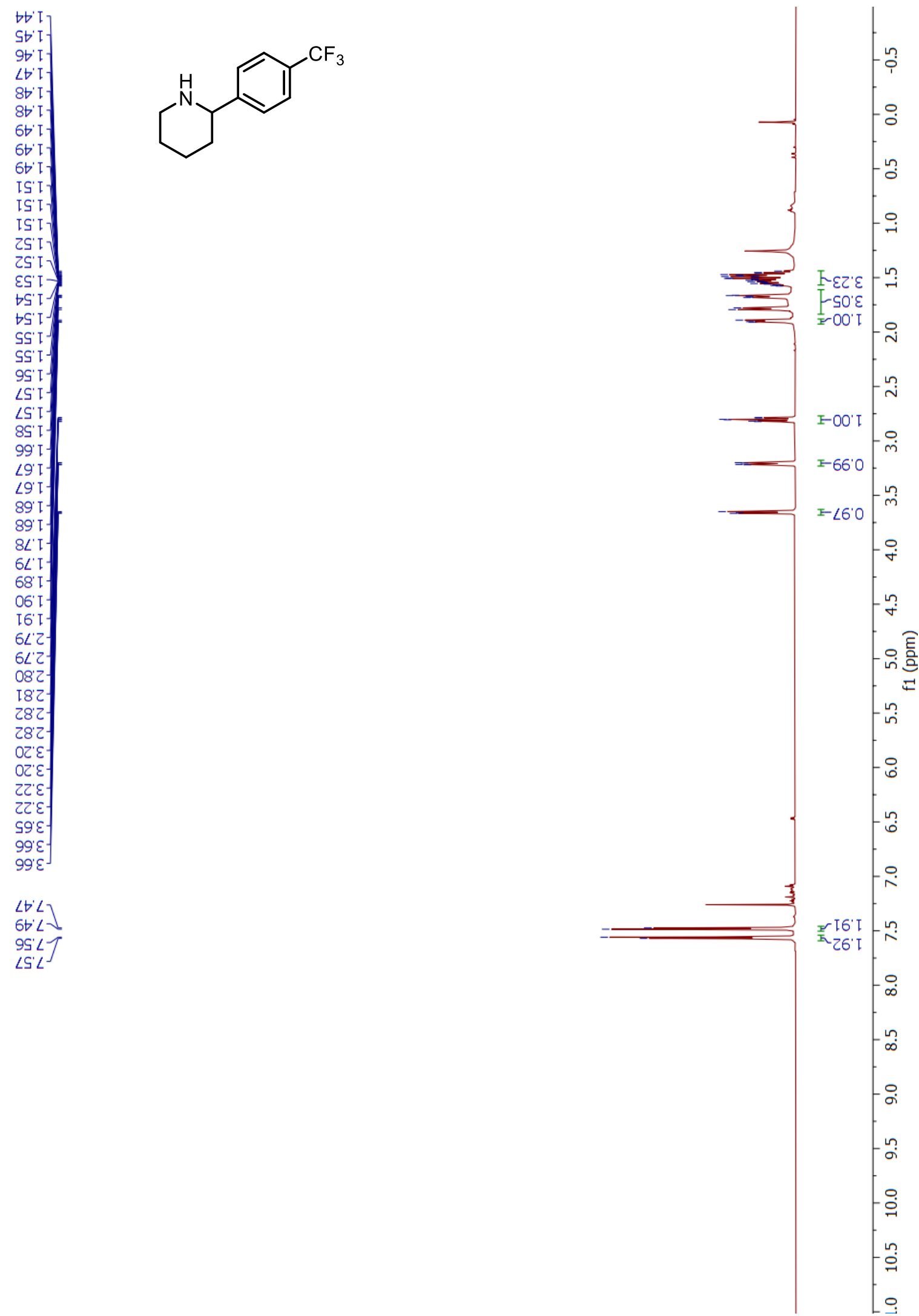
Figure S52. ${ }^{1} \mathrm{H}$ NMR (700 MHz) in $\mathrm{CDCl}_{3}$ 10c

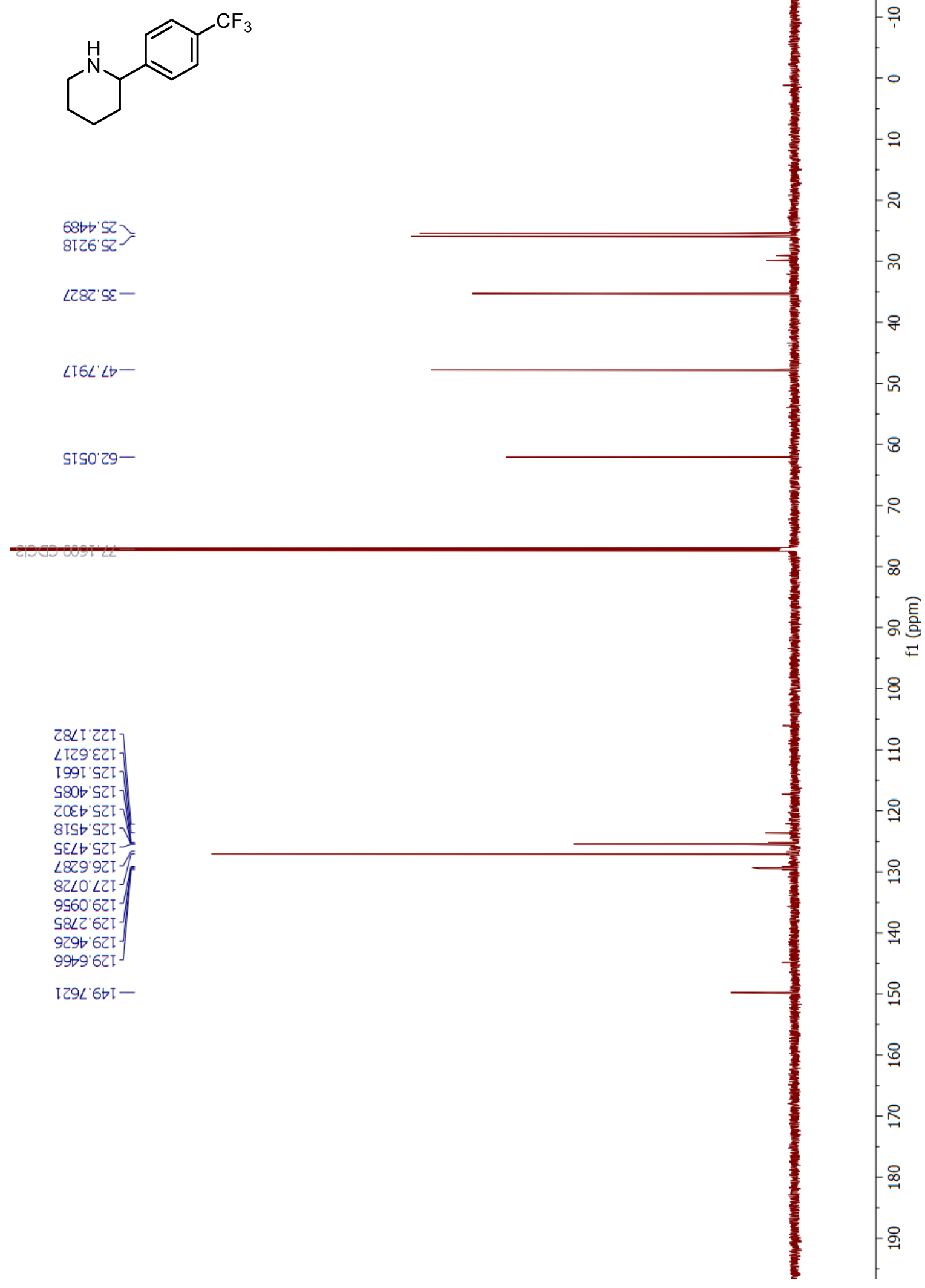


Figure S53. ${ }^{13} \mathrm{C}\left\{{ }^{1} \mathrm{H}\right\}$ NMR $(176 \mathrm{MHz})$ in $\mathrm{CDCl}_{3}$ 10c 


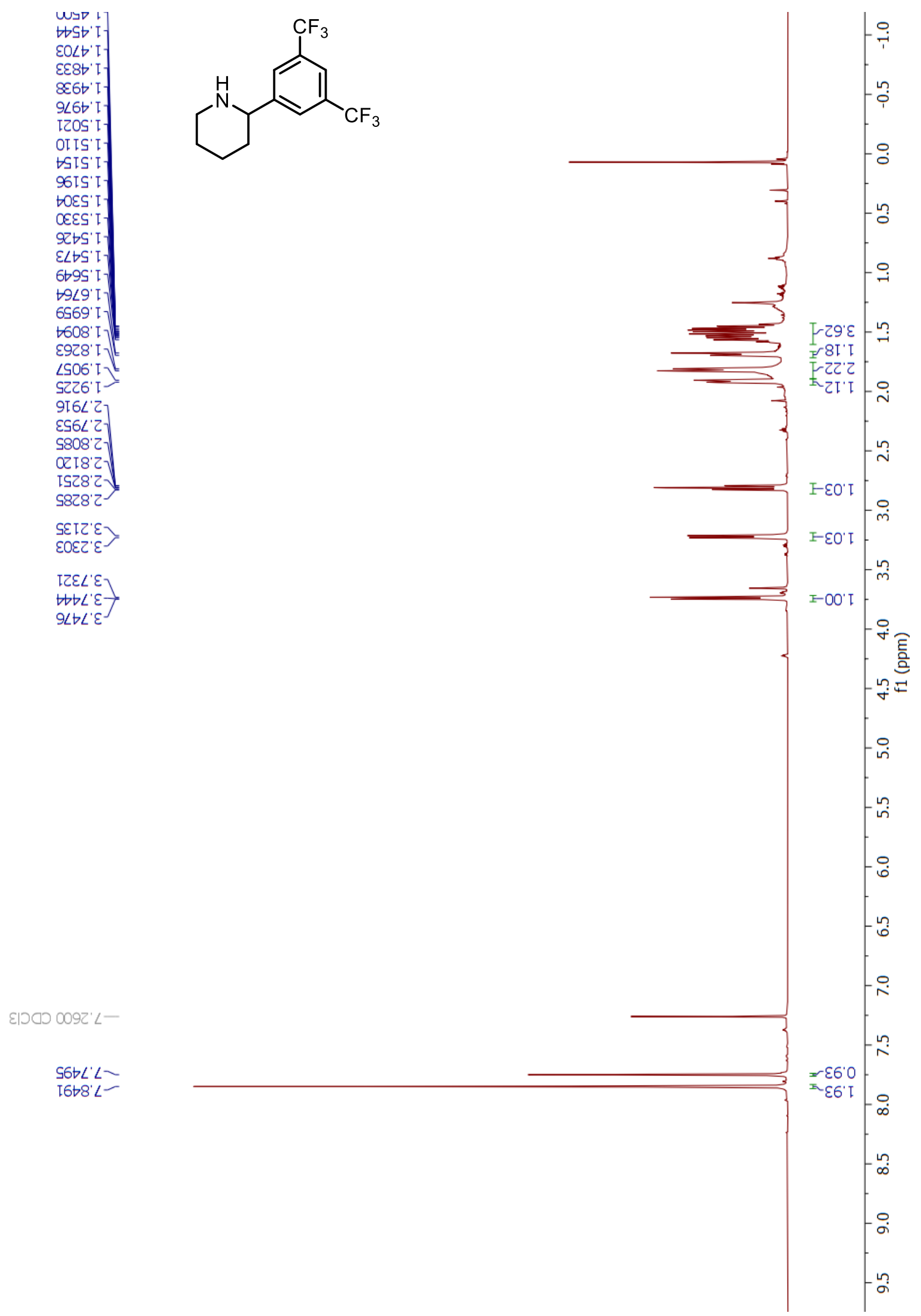

Figure S54. ${ }^{1} \mathrm{H}$ NMR (700 MHz) in $\mathrm{CDCl}_{3}$ 10d 


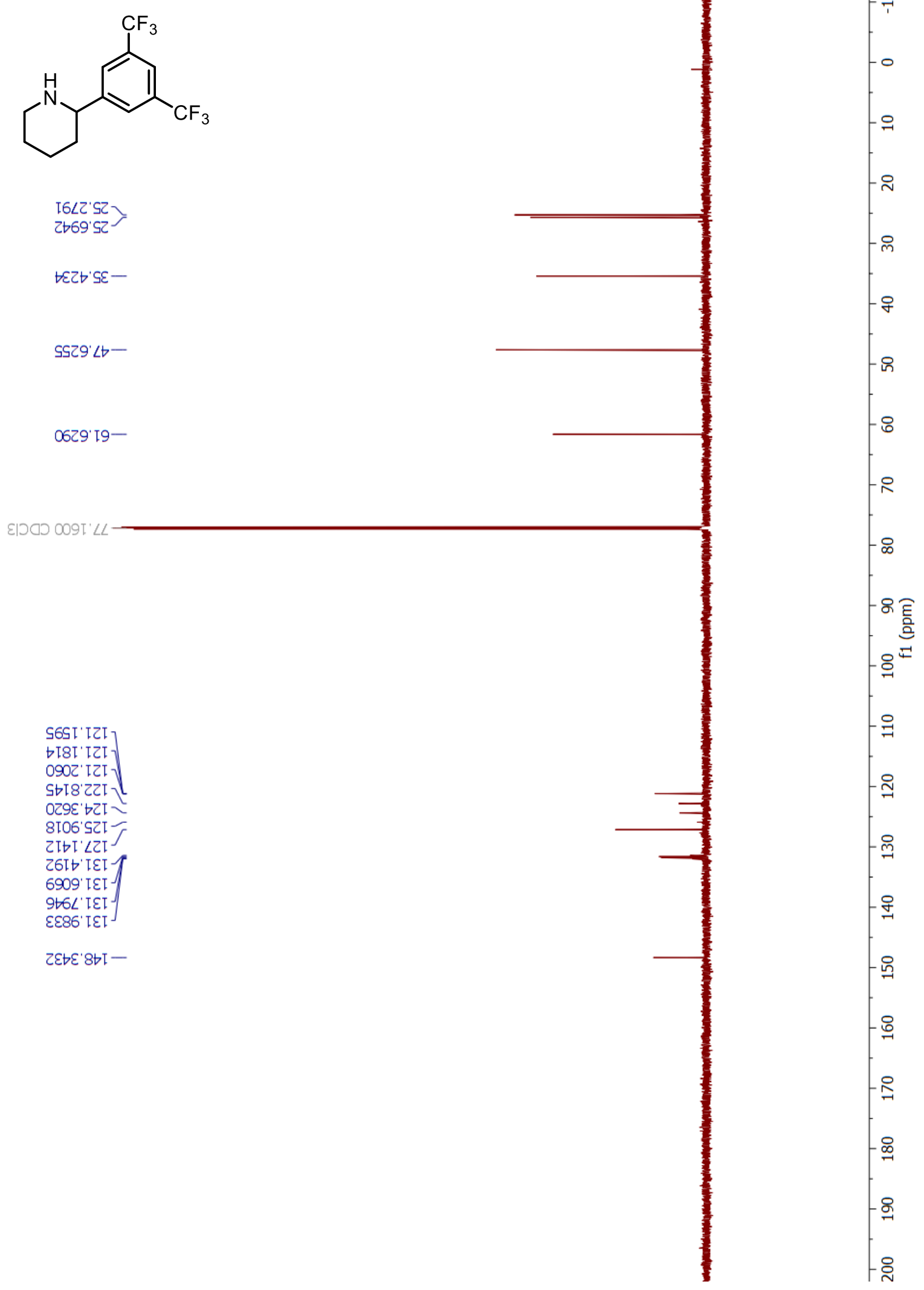

Figure S55. ${ }^{13} \mathrm{C}\left\{{ }^{1} \mathrm{H}\right\}$ NMR (176 MHz) in $\mathrm{CDCl}_{3}$ 10d 


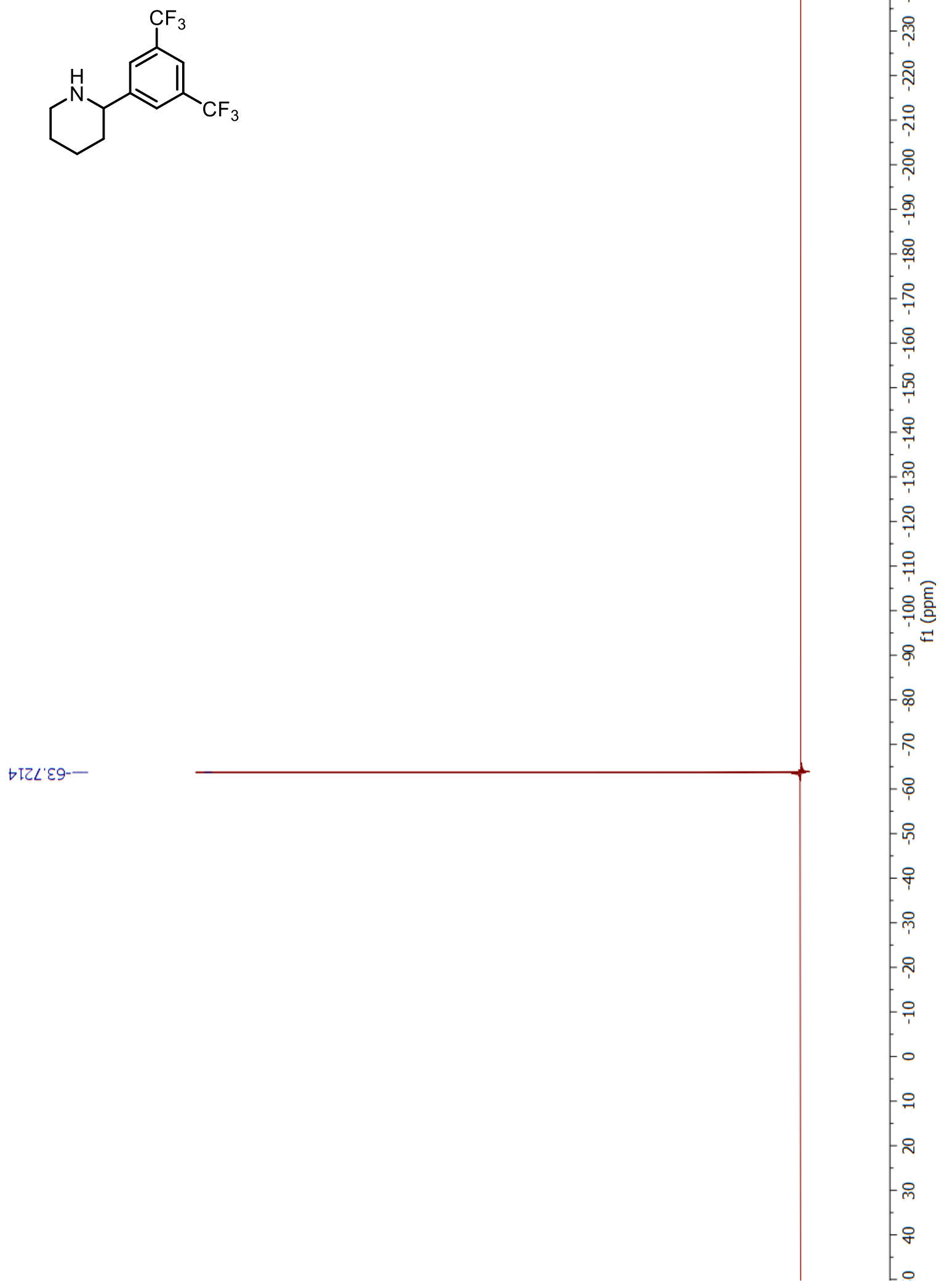

Figure S56. ${ }^{19} \mathrm{~F}$ NMR $(376 \mathrm{MHz})$ in $\mathrm{CDCl}_{3}$ 10d 